\title{
The contribution of Nintendo Wii Fit series in the field of health: a systematic review and meta-analysis
}

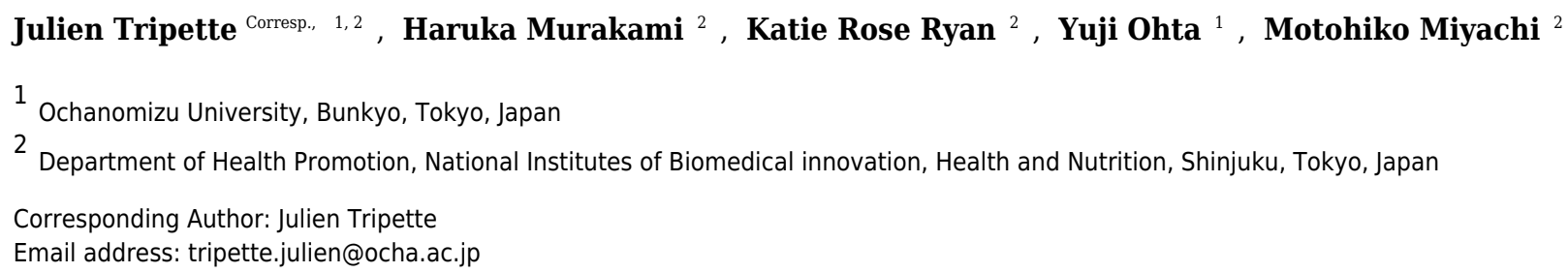

Background. Wii Fit was originally designed as a health and fitness interactive training experience for the general public. There are, however, many examples of Wii Fit being utilized in clinical settings. This article aims to identify the contribution of Wii Fit in the field of health promotion and rehabilitation by: 1 ) identifying the health-related domains for which the Wii Fit series has been tested, 2) clarifying the effect of Wii Fit in those identified health-related domains, and 3) quantifying this effect. Method. A systematic literature review was undertaken. The MEDLINE database and Games for Health Journal published content were explored using the search term "wii-fit". Occurrences resulting from manual searches on Google and material suggested by experts in the field were also considered. Included articles were required to have measurements from Wii Fit activities for at least one relevant health indicator. The effect of Wii Fit interventions was assessed using metaanalyses for the following outcomes: activity-specific balance confidence score, Berg balance score, and time-up-and-go test. Findings. 115 articles highlighted that the Wii Fit has been tested in numerous healthy and pathological populations. Out of these, only a few intervention studies have focused on the prevention of chronic diseases. A large proportion of the studies focus on balance training $(\mathrm{N}=55)$. This systematic review highlights several potential benefits of Wii Fit interventions and these positive observations are supported by meta-analyses data $(\mathrm{N}=25)$. For example, the Berg balance score and the time-up-and-go test respond to a similar extend to Wii Fit interventions compared with traditional training. Conclusions. Wii Fit has the potential to be used as a rehabilitation tool in different clinical situations. However, the current literature includes relatively few randomized controlled trials in each population. Further research is therefore required. 
1 The contribution of Nintendo Wii Fit series in the field of health: a systematic review and 2 meta-analysis

3

4 Julien Tripette ${ }^{1,2}$, Haruka Murakami ${ }^{2}$, Katie Rose Ryan ${ }^{2}$, Yuji Ohta ${ }^{1}$, Motohiko Miyachi ${ }^{2}$

5

$6{ }^{1}$ Ochanomizu University, Bunkyo, Tokyo, Japan

$7 \quad{ }^{2}$ National Institutes of Biomedical Innovation, Health and Nutrition, Department of Health

8 Promotion, Shinjuku, Tokyo, Japan

9

10 Corresponding author: Julien Tripette (tripette.julien@ocha.ac.jp) 
11

12

13

14

15

\section{Abstract}

Background. Wii Fit was originally designed as a health and fitness interactive training experience for the general public. There are, however, many examples of Wii Fit being utilized in clinical settings. This article aims to identify the contribution of Wii Fit in the field of health promotion and rehabilitation by: 1) identifying the health-related domains for which the Wii Fit series has been tested, 2) clarifying the effect of Wii Fit in those identified health-related domains, and 3) quantifying this effect. Method. A systematic literature review was undertaken. The MEDLINE database and Games for Health Journal published content were explored using the search term "wii-fit". Occurrences resulting from manual searches on Google and material suggested by experts in the field were also considered. Included articles were required to have measurements from Wii Fit activities for at least one relevant health indicator. The effect of Wii Fit interventions was assessed using meta-analyses for the following outcomes: activity-specific balance confidence score, Berg balance score, and time-up-and-go test. Findings. 115 articles highlighted that the Wii Fit has been tested in numerous healthy and pathological populations. Out of these, only a few intervention studies have focused on the prevention of chronic diseases. A large proportion of the studies focus on balance training $(\mathrm{N}=55)$. This systematic review highlights several potential benefits of Wii Fit interventions and these positive observations are supported by meta-analyses data $(\mathrm{N}=25)$. For example, the Berg balance score and the time-upand-go test respond to a similar extend to Wii Fit interventions compared with traditional training. Conclusions. Wii Fit has the potential to be used as a rehabilitation tool in different clinical situations. However, the current literature includes relatively few randomized controlled trials in each population. Further research is therefore required. 


\section{Introduction}

The past decade saw the emergence of home-based active video games (AVG), with the Wii (Nintendo Co. Ltd, Japan) being released in 2006, followed by the PlayStation Move (Sony Corp, Japan) and the Kinect (Microsoft, Washington, USA) in 2010. These systems take advantage of accelerometry and video camera-mediated motion detection technologies to track the player's movements and convert them into gaming commands. The Wii offers an original game modality with the Wii Balance board accessory, which can be used as a weighing scale or as a gamepad sensitive to body sway [1].

Among the home-based AVG, the well-known Wii Fit series (Nintendo, Japan) runs on the Wii console and consists of a combination of both serious and entertaining activities requiring body movement to fulfill gaming commands. The software displays various kinds of health metrics (body mass index, number of kilocalories burned over a given period) encouraging the players to improve their physical fitness. Whilst the Wii Fit was primarily designed to be used in homes by healthy individuals for health and fitness purposes, an overview of the literature indicates that physical therapists and physicians from different medical fields include the use of Wii Fit in their clinical practice. For instance, the National Stroke Audit: Rehabilitation Services Report recently indicated that $76 \%$ of Australian hospitals have a Wii console available to aid with the rehabilitation of stroke patients (the National Stroke Foundation, 2012 in [2]).

Many reviews have focused on AVG and their effects on health and describe mitigated outcomes $[3,4]$. However, the distinction between Wii Fit and other AVG was not always clear, resulting in the inability to ascertain an objective picture of the contribution from the Wii Fit. The goals for this systematic review are as follows: 
55 - Goal 1: Identifying the health-related domains (i.e. populations and clinical situations) in 56 which the Wii Fit series has already been tested or used. A scientific database search with 57 reasoned exclusion criteria was undertaken.

58 - Goal 2: Understanding the effect of Wii Fit in the identified populations (cf. Goal 1). A

59 qualitative systematic review of studies including Wii Fit interventions was performed, with 60 particular attention given to health and physical activity outcomes.

61 - Goal 3: When possible, quantification of the effect Wii Fit has on selected health-related 62 domains was achieved by conducting meta-analyses. 


\section{Methods}

64 Literature search

65 The selection process is summarized in a PRISMA flow diagram (Figure 1). Several strategies

66 were adopted: 1) The MEDLINE database was used to conduct a systematic search using the

67 following keywords: "wii fit", "wii-fit" and "wiifit" (occurrences: N=122). 2) The same

68 keywords were used to search for additional articles in the Mary Ann Liebert, Inc. Games and

69 Health Journal (N=121). 3) Additional peer-reviewed articles were identified during manual

70 searches via Google Search (Google Inc., California, USA) (N=1). 4) Articles suggested by

71 authors active in the field of AVG $(\mathrm{N}=10)$ or identified in the reference section of eligible papers

$72(\mathrm{~N}=46) .5)$ Only papers in English, French or Japanese were eligible for this review. The search

73 and data extraction were performed by two independent researchers (Murakami H and Tripette J)

74 and any discrepancies were resolved by a third contributor (Miyachi M). 


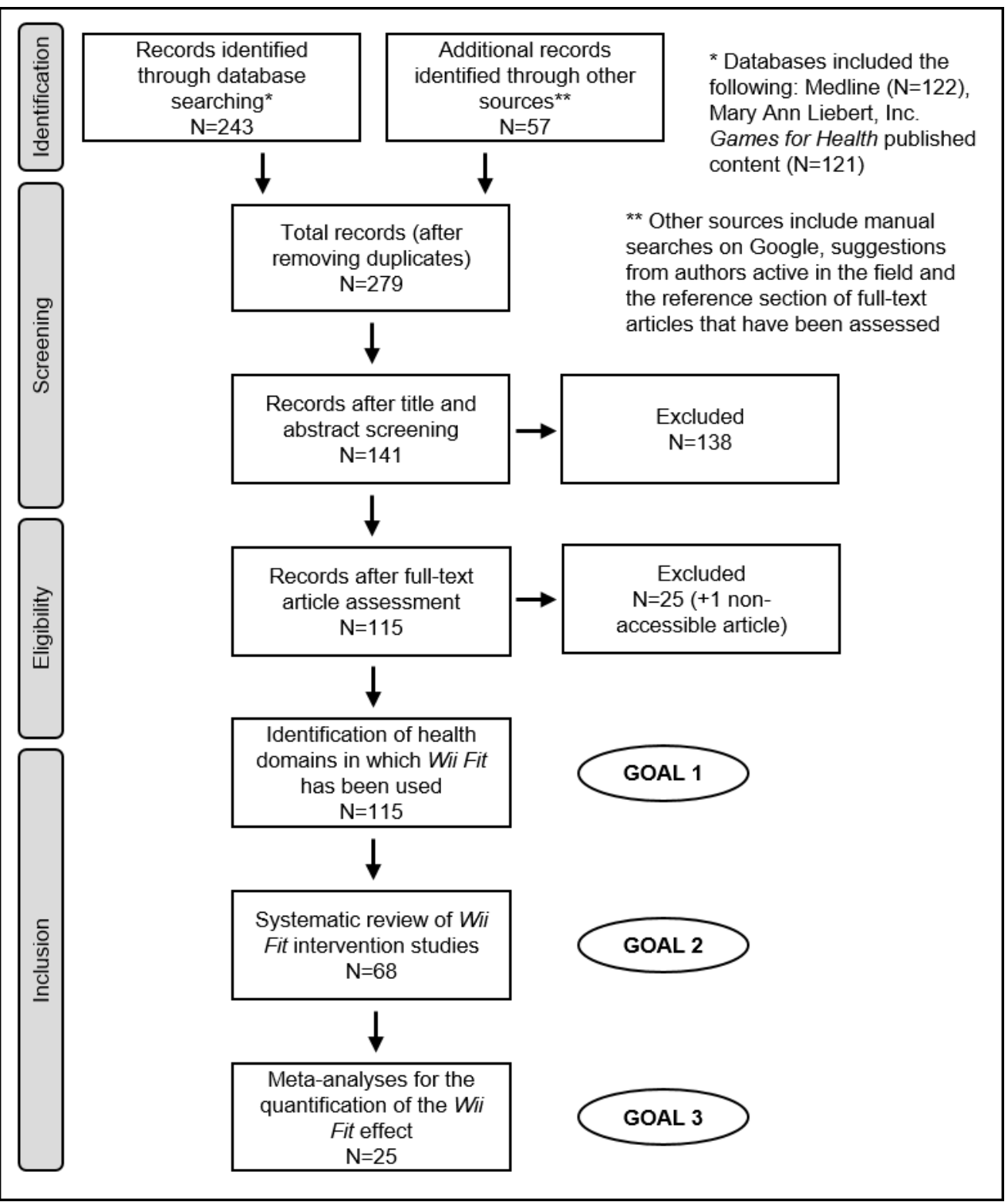

77 the meta-analyses. Details about exclusion criteria and the selection process can be found in

78 Table 1 and the Methods section. 
79 The literature search was completed in June 2015. A total of 200 articles were identified. In order

80 to meet the primary inclusion criteria, studies were required to: A) have a primary focus on any

81 software of the Wii Fit series, and B) focus on a recognized health issue. 279 articles were

82 screened after the identification and removal of 21 duplicates (Figure 1).

84 Goal 1: Identification of health domains

85 The exclusion criteria applied to identify medical domains in which the Wii Fit has already been

86 tested or used are described in Table 1. The identification process involved screening titles and

87 abstracts. The full texts were read when the abstracts provided insufficient details (Figure $1 \&$

88 Table 1). The results are shown in Table 2. 


\section{Table 1. Summary of exclusion criteria}

\begin{tabular}{|c|c|}
\hline Literature review stage & Exclusion criteria \\
\hline Screening & $\begin{array}{l}\text { 1) The study was not about any Wii Fit software. } \\
\text { 2) The study does not focus on any health issue. } \\
\text { 3) The article does not describe an original study. } \\
\text { 4) The article was one of the following: letter, commentaries, symposium } \\
\text { reports, interviews, conference abstracts, study protocols, reviews. } \\
\text { 5) The article has not been peer-reviewed. }\end{array}$ \\
\hline Goal 1 & $\begin{array}{l}\text { 1) Wii Fit was not the main component of the intervention. } \\
\text { 2) Wii Fit was used only to induce a stimulus without being the main object of } \\
\text { the study. } \\
\text { 3) The study focused on the Wii Fit avatar system rather than on its gaming } \\
\text { content. } \\
\text { 4) The article reports the development of software for the Wii Balance Board. } \\
\text { 5) The study focuses on the Wii Balance Board capabilities not on the Wii Fit } \\
\text { gaming content. }\end{array}$ \\
\hline Goal 2 & $\begin{array}{l}\text { 1) The article does not describe an intervention study. } \\
\text { 2) The study does not include an objective assessment for at least one health or } \\
\text { physical activity indicator, assessed quantitatively. } \\
\text { 3) The protocol includes less than } 5 \text { subjects or does not report average or } \\
\text { median values. }\end{array}$ \\
\hline Goal 3 & $\begin{array}{l}\text { 1) The study does not include numerical data for the activities-specific balance } \\
\text { confidence test (ABC), Berg balance score (BBS), or the time-up-and-go test } \\
\text { (TUG). }{ }^{\mathrm{a}} \\
\text { 2) The data reported for ABC, BBS and TUG were not mean } \pm \text { SD, or does not } \\
\text { allow the calculation of a mean values and the imputation of SD. }{ }^{\text {b }} \\
\text { 3) The magnitude of changes in ABC, BBS or TUG, were expected to be } \\
\text { important due to patients' initial condition and regardless of the chosen } \\
\text { rehabilitation program (e.g. post-surgery orthopedic patients). } \\
\text { 4) The population sample's average age was less than 5-years old. }{ }^{\mathrm{d}}\end{array}$ \\
\hline
\end{tabular}

${ }^{a}$ Examples of excluded studies for this selection criteria are $[5,6] .{ }^{b}$ Examples of excluded studies for this selection criteria are [7-10]. ${ }^{c}$ An example of an excluded study for this selection criteria is [11]. ${ }^{\mathrm{d}}$ An example of an excluded study for this selection criteria is [12]. SD: standard deviation. 
94 Goal 2: Systematic review, data extraction and synthesis

95 A qualitative systematic review was performed to understand the effect of Wii Fit in the

96 previously identified health domains. This study followed the 2009 PRISMA guidelines for the

97 conductance of systematic reviews and meta-analyses [13] (see, "S1 Checklist. PRISMA

98 checklist"). The exclusion criteria which were applied at this stage are described in Table 1.

99 The content of each eligible article was extracted according to the following protocol: 1) Study

100 identification (first author's name, year, and country), 2) methodological details (study design,

101 sample size, population characteristics, etc.), 3) activities used, 4) description of each identified

102 primary or secondary health and physical activity outcome, and 5) key findings (i.e. pre- and

103 post-intervention as well as differences between Wii Fit and control groups) (Table 3 \& 4).

Goal 3: Meta-analyses

106 The effects of Wii Fit were quantified for selected health-related domains. The most recurrent

107 outcomes noted were the activities-specific balance confidence test (ABC), Berg balance score

108 (BBS), and the time-up-and-go test (TUG). These three tests are frequently used to assess

109 patients' balance abilities [14-16]. ABC is usually administered by a health care professional

110 asking "How confident are you that you will not lose your balance or become unsteady when

111 you...” for 16 different situations (e.g., “...walk around the house?”, “...walk up or down

112 stairs”...). For each item, the participant should answer by expressing confidence in percentage

113 [14]. BBS is a scale able to measure balance in adults. The therapist asks participants to complete

11414 different tasks (e.g., "sitting to standing", "turning to look behind them"...) and evaluates

115 each of them using a 5-point score, ranging from 0 to 4 [15]. TUG is a simple measure of the 
116 time taken by a subject to stand up from a chair, walk a distance of 3 meters, turn, walk back to

117 the chair, and sit down (time is expressed in seconds) [16]. Firstly, pre- and post-intervention

118 meta-analyses were performed for each of these three outcomes. Secondly, Wii Fit vs. traditional

119 therapy meta-analyses were completed, which only included results from randomized control

120 (RCT) or two-arm trials. The exclusion criteria applied at this stage are described in Table 1.

121 Only studies that used the 3-meter version of the TUG test were included. Groups submitted to a 122 combination of Wii Fit activities and more traditional therapy exercises were excluded from the 123 pre- and post-intervention meta-analysis (e.g., [17-19]). The pre- and post-intervention effect 124 was calculated for the three selected outcomes. These meta-analyses used the mean difference 125 between the reported pre-intervention and post-intervention values. For the Wii Fit vs. traditional 126 therapy meta-analyses, the difference between the pre- and post- Wii Fit intervention changes 127 and the pre- and post- traditional intervention changes were used as inputs in the meta-analysis.

128 The variance imputation methods described by Follmann et al. were used to estimate the 129 standard deviations of effect size when the authors did not report them [20]. Heterogeneity 130 between studies was assessed using the homogeneity test. A fixed-effect model was used when 131 the $I^{2}$ statistic, which is the index of heterogeneity, was under $75 \%$. Sub-analyses were 132 conducted in patients and healthy subjects. For ABC, because only two studies included a 133 comparison between Wii Fit and traditional therapy [19, 21], only the pre- and post-intervention 134 meta-analysis was performed. The risk of bias in each individual study included in the Wii Fit vs. 135 traditional therapy meta-analysis was also assessed (Figure 2). Meta-regression analyses were 136 performed to assess the impact of intervention duration and volume (i.e. session

137 duration*number of session) on $\mathrm{ABC}, \mathrm{BBS}$ and TUG. $\mathrm{p}<0.05$ indicates statistical significance. 138 Meta-analysis was performed using STATA 12.1 (StataCorp, Texas, USA). 
Adequate sequence generation?

Adequate allocation concealment?

Similar baseline outcome measurements?

Similar baseline characteristics?

Incomplete outcome data?

Contamination between groups?

Blinding of outcome assessment?

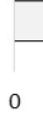

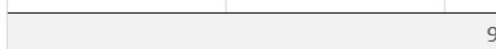

90

10

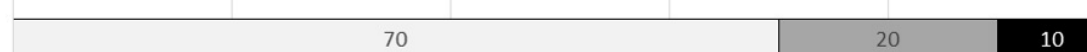

70

20

Figure 2. Assessment of risk of bias in individual studies included in the Wii Fit vs.

141 traditional therapy meta-analyses. The absence of ABC, BBS or TUG excluded de facto the

142 studies from the meta-analyses. Therefore the usually reported "reporting bias" was not included 143 in this assessment. No "other bias" was identified. 


\section{Results}

145 The literature search provided a total of 279 references of interest (Figure 1). Following the title

146 and abstract screening process 138 studies were discarded, as they did not meet the selection

147 criteria. 1 article was not accessible so was also discarded at this stage. An additional 25

148 references were removed after reading the full-text. Finally, 115 studies were included in the

149 qualitative analysis, covering an approximately 6-year period from July 2009 to June 2015.

150

151 Goal 1: Health domains and populations of interest

152 The 115 selected studies focused on Wii Fit as a novel tool to improve physical function, fitness

153 or health status. The content of the 115 articles was used to determine the different health

154 domains in which Wii Fit may have potential benefits (Table 2). 
Table 2. Wii Fit studies, health domains and populations of interest

\section{Juvenile population}

- Healthy children / adolescents ${ }^{\mathrm{a}}\left[2,23^{\mathrm{b}}, 24^{\mathrm{b}}, 25,26^{\mathrm{b}}\right]$

- Overweight children / adolescents $[24,26]^{\mathrm{b}}$

- Children with developmental delay [12, 27-30]

- Children with migraine [31]

- Children with Raynaud disease [32]

- Children with cystic fibrosis [33, 34]

- Children with cerebral palsy [35-38]

- Adolescents with autism spectrum disorders [39] Young adults \& middle-age adults

- Healthy adults ${ }^{\mathrm{a}, \mathrm{c}}\left[22,23^{\mathrm{b}}, 24^{\mathrm{b}}, 40,41-44,45^{\mathrm{b}}, 46^{\mathrm{b}}, 47-58\right]$

- Healthy women $\left[59,60,61^{\mathrm{b}}, 62,63^{\mathrm{d}}, 64\right]$

- Overweight adults $\left[24^{\mathrm{b}}, 45^{\mathrm{b}}, 46^{\mathrm{b}}, 61^{\mathrm{b}}, 65,66\right]$

- Depressed soldiers [67]

- Adults with drug dependency [68]

- Women with systemic lupus erythematosus $[69,70]$

- Adults with vestibular disorders [21,71 ${ }^{\mathrm{e}}$,

- Patients in orthopedic rehabilitation [11, 72-75]

- Amputees [76]

- COPD patients [77]

- Diabetic patients [78]

- Hemodialysis patients [79]

- Lower back pain patients [80]

- Adults with multiple sclerosis [81-86]

- $\quad$ Cancer patients ${ }^{\mathrm{f}}[87,88]$

- $\quad$ Stroke patients ${ }^{\mathrm{f}}[5,17,19,89-92]$

- Spinal cord injury patients [6]

- Healthy seniors $\left[7,8,9^{\mathrm{b}}, 93,94^{\mathrm{b}}, 95-98,99^{\mathrm{b}}, 100-106\right]$

- Senior with balance impairment $\mathrm{g}\left[8,9^{\mathrm{b}}, 10,18,99^{\mathrm{b}}, 107-109,94^{\mathrm{b}}, 110,111\right]$

- Seniors with cognitive impairments ${ }^{\mathrm{h}}[112-120]$

- Seniors with peripheral neuropathy [121]

- Other senior population [122]

${ }^{a}$ Not including overweight populations; ${ }^{b}$ Some papers focused on various populations may appear in several fields; ${ }^{\mathrm{c}}$ Not including studies that focus on healthy adult women only; ${ }^{\mathrm{d}}$ The study included healthy subjects but targeted women with urinary incontinence; e Patients with « other neurological disorders $»$ were included as well; ${ }^{\mathrm{f}}$ Includes both middle-age adults and seniors; g Includes subjects referred for rehabilitation, presenting a history of accidental falls, having fear of falling or described as frail or pre-frail; ' Includes both Parkinson's and Alzheimer's patients. Intervention studies eligible for inclusion in the systematic review are described in further detail in Table $3 \& 4$. 
164

165

166

167

168

169

170

171

172

173

174

175

176

177

178

179

180

181

182

183

184 185

186

\section{Goal 2: Systematic review of Wii-Fit interventions}

From the 115 selected Wii Fit articles, 68 were intervention studies and met the selection criteria for inclusion in the systematic qualitative review. Overall, these studies involved 2183 participants from both sexes (females: 1161, males: 844 , not specified: 178), with a wide age range (49 \pm 6 months $-86 \pm 6$ years $[12,100])$, and various medical conditions. Primary and secondary outcomes, intervention content, as well as observation period vary from study to study. The intervention durations vary from 2 to 20 weeks $[89,99]$, frequencies from 1 to 7 sessions per week (respectively, $[99,110]$ and $[64,78]$ ), and session time from 10 to 60 minutes (respectively, $[8,97]$ and $[72,81,99])$.

6 papers reported adverse effects: In young adults, light to moderate adverse effects (muscle soreness, pain, sprain, etc.) were observed [55]. Among seniors, hip strain, neck strain, lower back pain as well as one fall were reported [105, 108, 109]. In multiple sclerosis patients, knee pain and lower back pain were also reported [84]. Bower et al. (2014) observed a relatively high rate of falls in stroke patients (4 events over a group of 30 patients, [89]).

Table 3 describes the characteristics and main results from studies with a primary focus on the effects of Wii Fit interventions on physical activity level, physical fitness or patients' health status. Among 13 studies, 10 observed positive effects $[8,18,64,68,79,77-80,87,88,110]$ and 3 presented more contrasted results [24, 60, 77]. Interestingly, 4 intervention studies were conducted in patients with chronic diseases. They all reported a significant improvement in health status and well-being (chronic obstructive pulmonary disease, type two diabetes mellitus, chronic kidney disease, and lower back pain) [77-80]. 2 reports described Wii Fit interventions as both feasible and effective methods for improving the overall physical fitness, mobility and independence of senior subjects $[8,18,110]$. 
Table 3. Wii Fit interventions for health status and well-being improvement a

\begin{tabular}{|c|c|c|c|c|c|}
\hline $\begin{array}{l}\text { Authors } \\
\text { Year } \\
\text { Country }\end{array}$ & $\begin{array}{l}\text { Population } \\
\text { characteristics }\end{array}$ & Study design & $\begin{array}{l}\text { Wii Fit activities (or } \\
\text { other video games) }\end{array}$ & $\begin{array}{l}\text { Outcomes and } \\
\text { measures }\end{array}$ & $\begin{array}{l}\text { Key findings } \\
\text { \& Data used for the meta-analyses }\end{array}$ \\
\hline \multicolumn{6}{|c|}{ Healthy population } \\
\hline $\begin{array}{l}\text { Nitz et al. } \\
2010 \\
\text { Australia } \\
{[60]}\end{array}$ & $\begin{array}{l}\text { Women }(\mathrm{N}=10) \\
\text { Age range: } 30-60 \mathrm{yrs} \\
\text { Mean age: } 47 \pm 10 \mathrm{yrs}\end{array}$ & $\begin{array}{l}\text { Intervention } \\
1 \text { group } \\
\text { Duration: } 10 \text { wks (30 } \\
\text { min, } 2 \text { sessions /wk) } \\
\text { Location: Home } \\
\text { (supposedly) }\end{array}$ & $\begin{array}{l}\text { Not specified, possibly } \\
\text { all the Wii Fit's } \\
\text { activities }\end{array}$ & 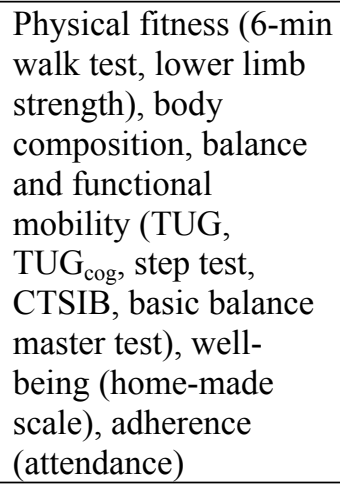 & $\begin{array}{l}\text { Improvement for some balance tests and } \\
\text { lower limb strength. The overall } \\
\text { attendance was } 70 \% \text {. } \\
\text { Adverse events: No } \\
\text { TUG (sec): } \\
\text { Wii Fit group } \\
\text { Pre-intervention: } \mathbf{4 . 9 3} \pm \mathbf{0 . 7 6} \\
\text { Post-intervention: } \mathbf{5 . 0 0} \pm \mathbf{0 . 7 3}\end{array}$ \\
\hline $\begin{array}{l}\text { Owens et } \\
\text { al. } \\
2011 \\
\text { USA } \\
{[24]}\end{array}$ & $\begin{array}{l}\text { Eight Families } \\
\text { (parents and children, } \\
F / M, N=13 / 8 \text { ) } \\
\text { Age range: } 8-44 \text { yrs }\end{array}$ & $\begin{array}{l}\text { Intervention } \\
1 \text { group (statistical } \\
\text { analysis: children } v s \text {. } \\
\text { adults) } \\
\text { Duration: } 13 \text { wks (no } \\
\text { further specifications: } \\
\text { naturalistic approach) } \\
\text { Location: Home }\end{array}$ & $\begin{array}{l}\text { Not specified (subjects } \\
\text { used the four } \\
\text { categories of } \\
\text { activities: yoga, } \\
\text { strength, aerobics, } \\
\text { balance) }\end{array}$ & $\begin{array}{l}\text { PA (accelerometry), } \\
\text { body composition, } \\
\text { balance (SOT), } \\
\text { physical fitness } \\
\text { ( } \mathrm{VO}_{2} \text { max, upper limb } \\
\text { strength, flexibility), } \\
\text { adherence (playing } \\
\text { time) }\end{array}$ & $\begin{array}{l}\text { No significant change was noted in most } \\
\text { of the physical fitness outcomes. Peak } \\
\mathrm{VO}_{2} \text { increased in children only. } \\
\text { Adherence declined over time. In } \\
\text { realistic conditions Wii Fit may not } \\
\text { provide sufficient stimulus for fitness } \\
\text { improvement. } \\
\text { Adverse events: No }\end{array}$ \\
\hline $\begin{array}{l}\text { Tripette et } \\
\text { al. } \\
2014 \\
\text { Japan } \\
{[64]}\end{array}$ & $\begin{array}{l}\text { Postpartum women } \\
(\mathrm{N}=34) \\
\text { Mean age: } 32 \pm 5 \mathrm{yrs}\end{array}$ & $\begin{array}{l}\text { Intervention (RCT) } \\
2 \text { groups: Wii Fit vs. } \\
\text { passive control } \\
\text { Duration: } 5 \text { wks ( } 30 \\
\text { min, daily) } \\
\text { Location: Home }\end{array}$ & $\begin{array}{l}\text { All activities included } \\
\text { in the Wii Fit Plus } \\
\text { software }\end{array}$ & $\begin{array}{l}\text { Body composition, } \\
\text { physical fitness } \\
\text { (flexibility and } \\
\text { strength), energy } \\
\text { intakes } \\
\text { (questionnaire), } \\
\text { adherence (playing } \\
\text { time) }\end{array}$ & $\begin{array}{l}\text { Women playing Wii Fit lost more weight } \\
\text { than their passive control counterpart. } \\
\text { They expended an average } 4700 \pm 2900 \\
\text { kcal playing Wii Fit and decrease their } \\
\text { energy intakes. } \\
\text { Adverse events: lower back pain }(\mathrm{N}=1) \text {, } \\
\text { ankle twist }(\mathrm{N}=1) \text { and wrist tendinitis } \\
(\mathrm{N}=1)\end{array}$ \\
\hline \multicolumn{6}{|c|}{ Chronic diseases } \\
\hline $\begin{array}{l}\text { Albores et } \\
\text { al. } \\
2013 \\
\text { USA }\end{array}$ & $\begin{array}{l}\text { COPD patients }(F / M, \\
N=14 / 6) \\
\text { Mean age: } 68 \pm 10 \mathrm{yrs}\end{array}$ & $\begin{array}{l}\text { Intervention } \\
1 \text { group } \\
\text { Duration: } 12 \text { wks ( } 30 \\
\text { min, daily) }\end{array}$ & $\begin{array}{l}\text { Aerobics: Basic Run, } \\
\text { Free Step; training } \\
\text { plus: Bird's Eye } \\
\text { Bull's-Eye, Obstacle }\end{array}$ & $\begin{array}{l}\text { Primary: physical } \\
\text { fitness (ESWT and } \\
\text { other tests) } \\
\text { Secondary: health }\end{array}$ & $\begin{array}{l}\text { Home-based Wii Fit training improved } \\
\text { physical fitness and overall health status } \\
\text { but not dyspnea in COPD patients. } \\
\text { Adverse events: No }\end{array}$ \\
\hline
\end{tabular}




\begin{tabular}{|c|c|c|c|c|c|}
\hline [77] & & Location: Home & Course & $\begin{array}{l}\text { status (CRQ-SR, } \\
\text { dyspnea assessment) }\end{array}$ & \\
\hline $\begin{array}{l}\text { Kempf \& } \\
\text { Martin } \\
2013 \\
\text { Germany } \\
{[78]}\end{array}$ & $\begin{array}{l}\text { T2DM patients }(F / M, \\
N=119 / 101) \\
\text { Age range: } 50-75 \mathrm{yrs} \\
\text { Mean age: } 61 \pm 8 \mathrm{yrs}\end{array}$ & $\begin{array}{l}\text { Intervention (RCT) } \\
2 \text { groups: Wii Fit- } \\
\text { traditional care } v \text { s. } \\
\text { traditional care-Wii Fit } \\
\text { Duration: } 12 \text { wks ( } 30 \\
\text { min/day) } \\
\text { Location: Home }\end{array}$ & $\begin{array}{l}\text { Not specified } \\
\text { (supposedly, all the } \\
\text { activities included in } \\
\text { Wii Fit Plus) }\end{array}$ & $\begin{array}{l}\text { Primary: glycemic } \\
\text { variations (HbA1c) } \\
\text { and various blood } \\
\text { markers } \\
\text { Secondary: body } \\
\text { composition, blood } \\
\text { pressure, PA } \\
\text { (questionnaire), } \\
\text { adherence (retention), } \\
\text { health status (SF-12, } \\
\text { PAID) and well-being } \\
\text { \& quality of life } \\
\text { (WHO-5, CESD) }\end{array}$ & $\begin{array}{l}\text { Subjects adhered to the Wii Fit } \\
\text { intervention (retention rate: } 80 \% \text { ). } \\
\text { Playing Wii Fit on a daily basis } \\
\text { significantly decreased HbAlc in T2DM } \\
\text { patients (-0.3 } \pm 1.1 \text { ). Fasting glucose, } \\
\text { weight, BMI, PA, as well as other well- } \\
\text { being outcomes were also improved. } \\
\text { Adverse events: No }\end{array}$ \\
\hline $\begin{array}{l}\text { Cho \& } \\
\text { Sohng } \\
2014 \\
\text { Korea } \\
{[79]}\end{array}$ & $\begin{array}{l}\text { Hemodialysis patients } \\
(F / M, N=18 / 28) \\
\text { Mean age: } 59 \pm 8 \text { yrs }\end{array}$ & $\begin{array}{l}\text { Intervention (RCT) } \\
2 \text { groups: Wii Fit vs. } \\
\text { passive control } \\
\text { Duration: } 8 \text { wks ( } 30 \\
\text { min, } 3 \text { sessions/wk) } \\
\text { Location: Hospital }\end{array}$ & $\begin{array}{l}\text { Yoga: Chair, Half } \\
\text { Moon, Standing Knee } \\
\text { (supposedly); } \\
\text { strength: Torso Twist, } \\
\text { Triceps Extension } \\
\text { (supposedly); balance: } \\
\text { Balance Bubble, } \\
\text { Tightrope Walk; } \\
\text { aerobics: Basic Steps } \\
\text { (supposedly), Hula } \\
\text { Hoop; training plus: } \\
\text { Big Top Juggling, } \\
\text { Bird's-Eye Bulls-Eye, } \\
\text { Rhythm Kung Fu, } \\
\text { Rhythm Parade } \\
\text { (+5 other activities } \\
\text { that were not explicitly } \\
\text { named) }\end{array}$ & $\begin{array}{l}\text { Physical Fitness (back } \\
\text { strength, handgrip, leg } \\
\text { strength, sit-and-reach, } \\
\text { single leg stance test), } \\
\text { body composition } \\
\text { (bioimpedancemetry), } \\
\text { fatigue (analogue } \\
\text { scale) }\end{array}$ & $\begin{array}{l}\text { Significant improvements were noted for } \\
\text { physical fitness, body composition and } \\
\text { fatigue in the Wii Fit group but not the } \\
\text { control group, suggesting that this } \\
\text { software could be used for health } \\
\text { promotion program in hemodialysis } \\
\text { patients. } \\
\text { Adverse events: No }\end{array}$ \\
\hline $\begin{array}{l}\text { Kim et al. } \\
2014 \\
\text { Korea } \\
{[80]}\end{array}$ & $\begin{array}{l}\text { Middle-aged women } \\
\text { with lower back pain } \\
(N=30) \\
\text { Mean age: } 47 \mathrm{yrs}\end{array}$ & $\begin{array}{l}\text { Intervention (RCT) } \\
2 \text { groups: Wii Fit vs. } \\
\text { traditional therapy } \\
\text { Duration: } 4 \text { wks ( } 30 \\
\text { min, } 3 \text { sessions } / \mathrm{wk}) \\
\text { Location: not } \\
\text { specified }\end{array}$ & $\begin{array}{l}\text { Yoga: Chair, Deep } \\
\text { Breathing, Half Moon, } \\
\text { Palm Tree, Sun } \\
\text { Salutation } \\
\text { (supposedly), Tree, } \\
\text { Warrior }\end{array}$ & $\begin{array}{l}\text { Pain (visual analogue } \\
\text { scale, pressure } \\
\text { algometry), disability } \\
\text { (ODI, RDQ, FABQ) }\end{array}$ & $\begin{array}{l}\text { Both interventions induced lower pain } \\
\text { and self-perceived disability. Wii Fit } \\
\text { induced significantly higher } \\
\text { improvements for all outcomes except } \\
\text { for deep tissue mechanical pain } \\
\text { sensitivity (pressure algometry). } \\
\text { Adverse event: No }\end{array}$ \\
\hline
\end{tabular}




\begin{tabular}{|c|c|c|c|c|c|}
\hline \multicolumn{6}{|c|}{ Drug dependency problems } \\
\hline $\begin{array}{l}\text { Cutter et al. } \\
2014 \\
\text { USA } \\
{[68]}\end{array}$ & $\begin{array}{l}\text { Opioid- or cocaine- } \\
\text { dependent subjects } \\
(F / M, N=17 / 12) \\
\text { Mean age: } 43 \pm 9 \text { yrs }\end{array}$ & $\begin{array}{l}\text { Intervention (RCT) } \\
2 \text { groups: Wii Fit vs. } \\
\text { sedentary video games } \\
\text { Duration: } 8 \text { wks (20- } \\
25 \text { min, } 5 \text { sessions/wk) } \\
\text { Location: } \text { Drug } \\
\text { rehabilitation center }\end{array}$ & $\begin{array}{l}\text { For each session, } \\
\text { subjects were invited } \\
\text { to choose, two } \\
\text { aerobics activities, } 1 \\
\text { yoga } \text { activity, } 1 \\
\text { balance activity and } 1 \\
\text { strength activity. }\end{array}$ & $\begin{array}{l}\text { Acceptability } \\
\text { (attendance, 4-item } \\
\text { questionnaire), } \\
\text { physical activity (in- } \\
\text { session energy } \\
\text { expenditure, IPAQ-L), } \\
\text { substance use (diary, } \\
\text { urine toxicology } \\
\text { screening), well-being } \\
\text { (PSS, BLSS, LOT) }\end{array}$ & $\begin{array}{l}\text { Both interventions showed high level of } \\
\text { acceptability, decreased substance use } \\
\text { and increased well-being. Wii Fit } \\
\text { participants reported high level of MVPA } \\
\text { at the end of the intervention period. } \\
\text { Adverse events: No }\end{array}$ \\
\hline \multicolumn{6}{|c|}{ Cancer patients (fatigue management) } \\
\hline $\begin{array}{l}\text { Hoffman et } \\
\text { al. b } \\
2013 \\
\text { USA } \\
\text { [87] }\end{array}$ & $\begin{array}{l}\text { Post-surgical non- } \\
\text { small lung cancer } \\
\text { patients }(F / M, N=5 / 2) \\
\text { Age range: } 53-73 \mathrm{yrs} \\
\text { Mean age: } 65 \pm 7 \mathrm{yrs}\end{array}$ & $\begin{array}{l}\text { Intervention (phase 1, } \\
\text { cf. phase } 2 \text { below) } \\
\text { 1 group } \\
\text { Duration: } 6 \text { wks (5-30 } \\
\text { min "walking with the } \\
\text { Wii (see elsewhere) + } \\
\text { 3-4 Wii Fit balance } \\
\text { activities, } 5 \\
\text { sessions/wk) } \\
\text { Location: Home }\end{array}$ & $\begin{array}{l}\text { Aerobics: "walking } \\
\text { with the Wii" (might } \\
\text { described an aerobics } \\
\text { - Free Run - activity } \\
\text { played by walking } \\
\text { instead of running); } \\
\text { balance: Ski Slalom, } \\
\text { Soccer Heading; } \\
\text { Training Plus: Driving } \\
\text { Range; and "other } \\
\text { activities" }\end{array}$ & $\begin{array}{l}\text { Acceptability } \\
\text { (questionnaire), } \\
\text { fatigue (BFI), Fatigue } \\
\text { management } \\
\text { (PSEFSM), balance } \\
\text { and functional } \\
\text { mobility (ABC, self- } \\
\text { efficacy for walking } \\
\text { duration instrument, } \\
\text { step-count) and } \\
\text { adherence (playing } \\
\text { time) }\end{array}$ & $\begin{array}{l}\text { Patients adhered to the Wii Fit } \\
\text { intervention, which was rated as } \\
\text { acceptable. Perceived efficacy for } \\
\text { balance and functional mobility } \\
\text { increased, perceived fatigue decreased, } \\
\text { and perceived self-efficacy for fatigue } \\
\text { self-management increased (No statistics } \\
\text { however). Light intensity home-based } \\
\text { exertion delivered via a game console } \\
\text { was effective for fatigue self- } \\
\text { management in cancer patients. } \\
\text { Adverse events: No }\end{array}$ \\
\hline $\begin{array}{l}\text { Hoffman et } \\
\text { al. } \\
2014^{\text {b }} \\
\text { USA } \\
\text { [88] }\end{array}$ & $\begin{array}{l}\text { Same as for phase } 1 \\
\text { (cf. above) }\end{array}$ & $\begin{array}{l}\text { Intervention (phase } 2, \\
\text { cf. phase } 1 \text { above) } \\
1 \text { group } \\
\text { Duration: } 10 \text { wks ( } 30 \\
\text { min "walking with the } \\
\text { Wii (see elsewhere) + } \\
3-4 \text { Wii Fit balance } \\
\text { activities, } 5 \\
\text { sessions/wk) } \\
\text { Location: Home } \\
\text { Note: phase } 1 \text { and } \\
\text { phase } 2 \text { together: } 16- \\
\text { wk intervention }\end{array}$ & $\begin{array}{l}\text { Same as for phase } 1 \\
\text { (cf. above) }\end{array}$ & $\begin{array}{l}\text { Same as for phase } 1 \\
\text { (cf. above) }\end{array}$ & $\begin{array}{l}\text { Positive outcomes noted at the end of } \\
\text { phase } 1 \text { (cf. the above) were maintained } \\
\text { or reinforced at the end of the phase } 2 . \\
\text { Light intensity home-based exertion } \\
\text { delivered via a game console was } \\
\text { effective for fatigue self-management in } \\
\text { cancer patients (even for those } \\
\text { undergoing an adjuvant therapy) for a } \\
\text { period as long as } 16 \text { weeks at least. } \\
\text { Adverse events: No } \\
\text { ABC (no unit): } \\
\text { Wii Fit group } \\
\text { Pre-intervention: } \mathbf{7 2 . 8} \pm \mathbf{2 0 . 5} \\
\text { Post-intervention: } \mathbf{8 8 . 9} \pm \mathbf{2 4 . 8}\end{array}$ \\
\hline
\end{tabular}




\begin{tabular}{|c|c|c|c|c|c|}
\hline $\begin{array}{l}\text { Yuen et al. } \\
2011 \\
\text { USA } \\
{[69]}\end{array}$ & $\begin{array}{l}\text { African American } \\
\text { women with systemic } \\
\text { lupus erythematosus } \\
(N=15) \\
\text { Age range: } 25-67 \mathrm{yrs} \\
\text { Mean age: } 47 \pm 14 \mathrm{yrs}\end{array}$ & $\begin{array}{l}\text { Intervention } \\
\text { 1 group } \\
\text { Duration: } 10 \mathrm{wks} \\
\text { (30min, } 2 \text { sessions/wk) } \\
\text { Location: Home }\end{array}$ & $\begin{array}{l}\text { Yoga, strength and } \\
\text { aerobics activities }\end{array}$ & $\begin{array}{l}\text { Primary: fatigue (FSS) } \\
\text { Secondary: anxiety } \\
\text { level, pain intensity, } \\
\text { body composition, } \\
\text { step-count, physical } \\
\text { fitness, adherence }\end{array}$ & $\begin{array}{l}\text { Fatigue, anxiety and pain were reduced. } \\
\text { Body composition and physical fitness } \\
\text { improved. Good adherence. } \\
\text { Adverse events: No }\end{array}$ \\
\hline \multicolumn{6}{|c|}{ Seniors } \\
\hline $\begin{array}{l}\text { Chan et al. } \\
2012 \\
\text { China } \\
{[110]}\end{array}$ & $\begin{array}{l}\text { Elderly referred for } \\
\text { rehabilitation }(F / M, \\
N=22 / 8,13 \text { of them } \\
\text { having acquired } \\
\text { neurological disorders }) \\
\text { Mean age: } 80 \pm 7 \mathrm{yrs}\end{array}$ & $\begin{array}{l}\text { Intervention } \\
1 \text { group (+ comparison } \\
\text { with a historic pool of } \\
60 \text { patients) } \\
\text { Duration: } 5-9 \text { wks }(10 \\
\text { min, } 1-2 \text { sessions/wk, } \\
\text { total of } 8 \text { Wii Fit } \\
\text { sessions) } \\
\text { Location: Geriatric } \\
\text { center }\end{array}$ & Aerobics: 2P Run & $\begin{array}{l}\text { Primary: feasibility } \\
\text { (Borg scale, HR), } \\
\text { adherence (playing } \\
\text { time), acceptability } \\
\text { (questionnaire) } \\
\text { Secondary: functional } \\
\text { ability (FIM) }\end{array}$ & $\begin{array}{l}\text { Participants completed an average of } 72 \\
\pm 7 \text { min of Wii Fit during their 5-9-wk } \\
\text { rehabilitation period (instructions: about } \\
80 \text { min). No difference was noted in } \\
\text { exertion rate between the Wii Fit activity } \\
\text { and a traditional arm ergometer exercise. } \\
\text { Wii Fit participants exhibited a higher } \\
\text { improvement in functional abilities } \\
\text { compared to historic controls and wanted } \\
\text { to continue the game at home. } \\
\text { Adverse events: No }\end{array}$ \\
\hline $\begin{array}{l}\text { Daniel } \\
\text { USA } \\
2012 \\
{[18]}\end{array}$ & $\begin{array}{l}\text { Pre-frail elderly }(F / M \text {, } \\
N=14 / 9) \\
\text { Mean age }: 77 \pm 5 \mathrm{yrs}\end{array}$ & $\begin{array}{l}\text { Intervention }(\mathrm{RCT}) \\
3 \text { groups : passive } \\
\text { control vs. seated } \\
\text { exercise training } v s . \\
\text { Wii Fit }+ \text { weight vest } \\
\text { Duration: } 15 \text { wks ( } 45 \\
\text { min, } 3 \text { sessions/wks) } \\
\text { Location: Laboratory }\end{array}$ & $\begin{array}{l}\text { Not specified } \\
\text { (supposedly, all } \\
\text { activities included in } \\
\text { Wii Fit and Wii } \\
\text { Sports) }\end{array}$ & $\begin{array}{l}\text { Physical fitness (SFT, } \\
\text { CHAMPS) body } \\
\text { composition, balance } \\
\text { \& functional mobility } \\
\text { (ABC, LLFDI, 8-feet } \\
\text { TUG), adherence } \\
\text { (attendance) }\end{array}$ & $\begin{array}{l}\text { Authors described an improvement in } \\
\text { physical fitness and balance confidence } \\
\text { in the Wii Fit group, but no statistical } \\
\text { significance was indicated. Same } \\
\text { attendance rate }(86 \%) \text { in both seated } \\
\text { exercising and Wii Fit groups. } \\
\text { Adverse events: No }\end{array}$ \\
\hline
\end{tabular}

a When balance outcomes were included concomitantly with other outcomes, and were not described as a primary outcome alone, the study was only included in Table 3. ${ }^{\mathrm{b}}$ Hoffman et al. (2013 \& 2014) report results from two different phases of the same project [87, 88].

\%: percentage; ABC: activities-specific balance confidence scale; BFI: brief fatigue inventory; BLSS: brief life satisfaction scale; BMI: body mass index; CESD: center for epidemiologic studies depression scale; CHAMPS: community healthy activities model program for seniors; COPD:

chronic obstructive pulmonary disease; CRQ-SR: chronic respiratory questionnaire; CTSIB: clinical test of sensory interaction and balance ; EE: energy expenditure; ESWT: endurance shuttle walk test; FABQ: fear avoidance beliefs questionnaire; FIM: functional independence measure; FFS: Fatigue Severity Scale; HR: hear rate (beats/min); IMI: Intrinsic Motivation Inventory; LLFDI: late life function and disability index; IPAQ-L: physical activity questionnaire-long version; LOT: life orientation test; METs: metabolic equivalent; MVPA: moderate-to-vigorous physical activity; PSEFSM: perceived self-efficacy for fatigue self-management; RPP: rate pressure product; PAID: problem areas in diabetes scale; sec: second; SEES: subjective exercise experience scale; SFT: senior fitness test; PA: physical activity; PACES: physical activity and exercise questionnaire; ODI: Oswestry low-back pain disability index; PSS: perceived stress scale; RDQ: Roland Morris disability questionnaire; SF-12: 
short form-12 health survey; SOT: sensory organization test; TD2M: type 2 diabetes mellitus; TUG: time up and go; VPA: vigorous physical activity; $\mathrm{VO}_{2}$ : oxygen consumption; $\mathrm{VO}_{2}$ max: maximal oxygen consumption; WHO-5: five-item WHO well-being index.

201 For the same test, unit may vary from one paper to another. 
202 For each population, Table 4 summarizes study characteristics and the main results for protocols

203 with a primary focus on the effect of Wii Fit intervention on balance activities and related

204 physical functions. Overall, the qualitative review of these studies supports a positive effect of

205 Wii Fit interventions on balance outcomes. Among the 55 studies, 50 observed a positive effect

206 of Wii Fit on at least one parameter [5-7, 9-12, 17, 19, 21, 27-31, 36, 37, 42, 43, 52, 57, 72, 74,

$20775,81,82,84,86,90,92,93,95,98-102,105,106,108,109,111,113-120]$. There were

208 numerous examples were balance-related parameters improved to a similar or even higher extent

209 when using Wii Fit compared to traditional therapies $[5,11,17-19,21,42,43,52,72,74,75,81$,

$21096,90,95,99,105,111,116,120]$. Only 5 papers described contrasted results or expressed

211 some reservations about the ability of the software to induce benefits in balance skills $[8,35,58$,

$21289,97]$, with 3 of these studies being conducted in healthy populations [8, 58, 97]. 


\begin{tabular}{|c|c|c|c|c|c|}
\hline $\begin{array}{l}\text { Authors } \\
\text { Year } \\
\text { Country }\end{array}$ & $\begin{array}{l}\text { Population } \\
\text { characteristics }\end{array}$ & Study design & $\begin{array}{l}\text { Wii Fit activities (or } \\
\text { other video games) }\end{array}$ & $\begin{array}{l}\text { Outcomes and } \\
\text { measures }\end{array}$ & $\begin{array}{l}\text { Key findings } \\
\text { \& Data used for the meta-analyses }\end{array}$ \\
\hline \multicolumn{6}{|c|}{ Healthy young adults or middle-aged adults } \\
\hline $\begin{array}{l}\text { Gioftsidou et } \\
\text { al. } \\
2013 \\
\text { Greece } \\
{[42]}\end{array}$ & $\begin{array}{l}\text { Healthy young adults } \\
(F / M, N=18 / 22) \\
\text { Age range: } 20-22 \text { yrs } \\
\text { Mean age: } 20 \pm 1 \text { yrs }\end{array}$ & $\begin{array}{l}\text { Intervention (RCT) } \\
2 \text { groups: Wii Fit vs. } \\
\text { BOSU ball-based } \\
\text { therapy } \\
\text { Duration: } 8 \text { wks (14 } \\
\text { min, } 2 \text { sessions/wk) } \\
\text { Location: Laboratory } \\
\text { (supposedly) }\end{array}$ & $\begin{array}{l}\text { Balance: Balance } \\
\text { Bubble, Penguin } \\
\text { Slide, Snowboard } \\
\text { Slalom, Ski Slalom, } \\
\text { Soccer Heading, } \\
\text { Table Tilt; Training } \\
\text { plus: Balance Bubble } \\
\text { Plus, Skateboard } \\
\text { Arena, Table Tilt Plus }\end{array}$ & $\begin{array}{l}\text { Balance (single leg } \\
\text { stance tests and } \\
\text { various indexes using } \\
\text { Biodex system) }\end{array}$ & $\begin{array}{l}\text { Balance improvements for both BOSU } \\
\text { ball and Wii Fit intervention. Only one } \\
\text { test (the balance board anterior-posterior } \\
\text { single-limb stance test) showed greater } \\
\text { improvement for the BOSU ball } \\
\text { training. } \\
\text { Adverse events: No }\end{array}$ \\
\hline $\begin{array}{l}\text { Melong \& } \\
\text { Keats } \\
2013 \\
\text { Canada } \\
{[43]}\end{array}$ & $\begin{array}{l}\text { Healthy young adults } \\
(F / M, N=12 / 8) \\
\text { Mean age: } 20 \pm 1 \text { yrs }\end{array}$ & $\begin{array}{l}\text { Intervention (RCT) } \\
2 \text { groups: Wii Fit vs. } \\
\text { BOSU ball-based } \\
\text { therapy } \\
\text { Duration: } 4 \text { wks }(20 \\
\text { min, } 3 \text { sessions/wk) } \\
\text { Location: Laboratory }\end{array}$ & $\begin{array}{l}\text { Balance: Ski Jump, } \\
\text { Ski Slalom, Soccer } \\
\text { Heading, Table Tilt (+ } \\
\text { a other activities } \\
\text { played with the Wii } \\
\text { balance board) }\end{array}$ & $\begin{array}{l}\text { Primary: adherence } \\
\text { (attendance and } \\
\text { playing time) } \\
\text { Secondary: enjoyment } \\
\text { (PACES), balance } \\
\text { (stabilometry) }\end{array}$ & $\begin{array}{l}\text { Balance improvement were noted in } \\
\text { both groups. While the Wii Fit group } \\
\text { showed higher levels of enjoyment, this } \\
\text { did not lead to a significantly higher } \\
\text { attendance or playing time. This study } \\
\text { may have been underpowered. } \\
\text { Adverse events: No }\end{array}$ \\
\hline $\begin{array}{l}\text { Lee et al. } \\
2014 \\
\text { Korea } \\
{[52]}\end{array}$ & $\begin{array}{l}\text { Healthy young adults } \\
(N=24) \\
\text { Mean age: } 20 \pm 1 \text { yrs }\end{array}$ & $\begin{array}{l}\text { Intervention (RCT) } \\
2 \text { groups: Wii Fit vs. } \\
\text { indoor horseback } \\
\text { riding exercise } \\
\text { Duration: } 6 \text { wks }(25 \\
\text { min, } 3 \text { sessions } / \mathrm{wk}) \\
\text { Location: not } \\
\text { specified (« indoor ») }\end{array}$ & $\begin{array}{l}\text { Balance: Balance } \\
\text { Bubble, Ski Slalom, } \\
\text { Table Tilt }\end{array}$ & $\begin{array}{l}\text { Balance (dynamic } \\
\text { tests using Biodex } \\
\text { system: } \\
\text { anteroposterior, } \\
\text { mediolateral, and } \\
\text { overall stability) }\end{array}$ & $\begin{array}{l}\text { Both the Wii Fit and indoor horseback } \\
\text { riding programs induce significant } \\
\text { improvement in all three dynamic } \\
\text { balance tests. } \\
\text { Adverse events: No }\end{array}$ \\
\hline $\begin{array}{l}\text { Cone et al. } \\
2015 \\
\text { USA } \\
{[57]}\end{array}$ & $\begin{array}{l}\text { Healthy young adults } \\
(F / M, N=16 / 24) \\
\text { Age range: } 18-35 \text { yrs } \\
\text { Mean age: } 23 \pm 3 \text { yrs }\end{array}$ & $\begin{array}{l}\text { Intervention (RCT) } \\
2 \text { groups: Wii Fit vs. } \\
\text { passive control } \\
\text { Duration: } 6 \text { wks (30- } \\
45 \text { min, } 2-4 \\
\text { sessions/wk) } \\
\text { Location: Laboratory } \\
\text { (supposedly) }\end{array}$ & $\begin{array}{l}\text { Balance: Balance } \\
\text { Bubble, Penguin } \\
\text { Slide, Snowboard } \\
\text { Slalom, Ski Slalom, } \\
\text { Soccer Heading, } \\
\text { Table Tilt, Tightrope } \\
\text { Walk }\end{array}$ & Balance (SOT, LOF) & $\begin{array}{l}\text { Significantly higher improvements in } \\
\text { both LOS and SOT scores were noted } \\
\text { for the Wii Fit group. Because those } \\
\text { tests respectively focus on dynamic } \\
\text { stability and sensory weighting, the } \\
\text { results suggest that individuals with } \\
\text { vestibular system alterations or dynamic } \\
\text { balance control impairments may } \\
\text { benefit from Wii Fit training. }\end{array}$ \\
\hline
\end{tabular}




\begin{tabular}{|c|c|c|c|c|c|}
\hline & & & & & Adverse events: No \\
\hline $\begin{array}{l}\text { Naumann et } \\
\text { al. } \\
2015 \\
\text { Germany } \\
{[58]}\end{array}$ & $\begin{array}{l}\text { Healthy young adults } \\
(F / M, N=29 / 8) \\
\text { Age range: } 20-34 \text { yrs } \\
\text { Mean age: } 23 \pm 3 \text { yrs }\end{array}$ & $\begin{array}{l}\text { Intervention (RCT) } \\
3 \text { groups: Wii Fit vs. } \\
\text { MFT Challenge } \\
\text { Disc }{ }^{\circledR} \text { vs. passive } \\
\text { control } \\
\text { Duration: } 4 \text { wks }(30 \\
\text { min, } 3 \text { sessions/wk) + } \\
\text { follow-up after } 4 \\
\text { weeks } \\
\text { Location: Laboratory } \\
\text { (supposedly) }\end{array}$ & $\begin{array}{l}\text { Balance: Balance } \\
\text { Bubble, Ski Slalom, } \\
\text { Snowboard Slalom, } \\
\text { Table Tilt }\end{array}$ & $\begin{array}{l}\text { Balance (game scores, } \\
\text { single- or two-leg } \\
\text { stance COP excursion) }\end{array}$ & $\begin{array}{l}\text { The performance on trained games } \\
\text { increased in both intervention groups. } \\
\text { No changes were noted for the COP } \\
\text { excursion tests. Similarly, the Wii Fit } \\
\text { group did not show any increase in } \\
\text { MFT Challenge Disc } \AA \text { scores, and vice- } \\
\text { versa. These data suggest that the } \\
\text { training effect of Wii Fit was highly } \\
\text { specific and may not be transferred to } \\
\text { real life balance-related tasks. } \\
\text { Adverse events: No }\end{array}$ \\
\hline \multicolumn{6}{|c|}{ Healthy seniors } \\
\hline $\begin{array}{l}\text { Williams et al. } \\
2011 \\
\text { USA } \\
{[93]}\end{array}$ & $\begin{array}{l}\text { Elderly }(F / M, \\
N=18 / 4) \\
\text { Age range: } 74-84 \mathrm{yrs} \\
\text { Mean age: } 84 \pm 5 \mathrm{yrs}\end{array}$ & $\begin{array}{l}\text { Intervention } \\
1 \text { group } \\
\text { Duration: } 4 \text { wks }(20 \\
\text { min, } 1 \text { session/wk) } \\
\text { Location: Geriatric } \\
\text { center-based }\end{array}$ & $\begin{array}{l}\text { Balance and aerobics } \\
\text { activities }\end{array}$ & Balance (BBS) & $\begin{array}{l}\text { Wii Fit induced improvement in balance } \\
\text { skills. The post-intervention BBS scores } \\
(49 \pm 5) \text { were significantly higher than } \\
\text { pre-intervention scores }(39 \pm 6) \text {. } \\
\text { Adverse events: No } \\
\text { BBS (no unit): } \\
\text { Wii Fit group } \\
\text { Pre-intervention: } \mathbf{3 9 . 4 1} \pm \mathbf{6 . 2 8} \\
\text { Post-intervention: } \mathbf{4 8 . 5 5} \pm \mathbf{4 . 5 8}\end{array}$ \\
\hline $\begin{array}{l}\text { Bateni } \\
2012 \\
\text { USA } \\
{[95]}\end{array}$ & $\begin{array}{l}\text { Elderly }(F / M, N=9 / 8) \\
\text { Age range: } 53-91 \mathrm{yrs} \\
\text { Mean age: } 73 \pm 14 \mathrm{yrs}\end{array}$ & $\begin{array}{l}\text { Intervention (RCT) } \\
3 \text { groups: Wii Fit vs. } \\
\text { Wii Fit + traditional } \\
\text { physical therapy } v \text {. } \\
\text { traditional physical } \\
\text { therapy alone } \\
\text { Duration: } 4 \text { wks ( } 15 \\
\text { min - estimation, } 3 \\
\text { sessions/wk) } \\
\text { Location: } \\
\text { Rehabilitation center }\end{array}$ & $\begin{array}{l}\text { Balance: Balance } \\
\text { Bubble, Ski Jump, Ski } \\
\text { Slalom; training plus: } \\
\text { Table Tilt plus }\end{array}$ & $\begin{array}{l}\text { Balance (BBS and Wii } \\
\text { Fit Balance Bubble } \\
\text { score) }\end{array}$ & $\begin{array}{l}\text { Improvements in both BBS and Balance } \\
\text { Bubble score in all } 3 \text { groups were } \\
\text { observed. However, subjects who } \\
\text { underwent traditional therapy exercises } \\
\text { performed better at the BBS compared } \\
\text { to subjects who only play Wii Fit alone. } \\
\text { Adverse events: No }\end{array}$ \\
\hline $\begin{array}{l}\text { Franco et al. } \\
2012 \\
\text { USA } \\
\text { [97] }\end{array}$ & $\begin{array}{l}\text { Elderly }(F / M, \\
N=25 / 7) \\
\text { Mean age: } 78 \pm 6 \text { yrs }\end{array}$ & $\begin{array}{l}\text { Intervention (RCT) } \\
3 \text { groups: Wii Fit }+ \\
\text { strength training } v s \text {. } \\
\text { traditional balance } \\
\text { training } v \text { s. passive } \\
\text { control }\end{array}$ & $\begin{array}{l}\text { Balance: Ski Jump, } \\
\text { Ski Slalom, Soccer } \\
\text { Heading, Table Tilt, } \\
\text { Tightrope Walk }\end{array}$ & $\begin{array}{l}\text { Balance and gait } \\
\text { (BBS, Tinetti test), } \\
\text { functional health and } \\
\text { well-being (SF-36), } \\
\text { enjoyment (home- } \\
\text { made questionnaire) }\end{array}$ & $\begin{array}{l}\text { Wii Fit did not induce any balance and } \\
\text { gait improvements. Same outcomes } \\
\text { were observed in the traditional training } \\
\text { group. Subjects playing Wii Fit reported } \\
\text { high level of enjoyment. } \\
\text { Adverse events: No }\end{array}$ \\
\hline
\end{tabular}




\begin{tabular}{|c|c|c|c|c|c|}
\hline & & $\begin{array}{l}\text { Duration: } 3 \text { wks (10- } \\
15 \text { min, } 2 \\
\text { sessions/wk) } \\
\text { Location: Community } \\
\text { dwelling }\end{array}$ & & $\begin{array}{l}\text { and adherence } \\
\text { (playing time) }\end{array}$ & $\begin{array}{l}\text { BBS (no unit): } \\
\text { Wii Fit group } \\
\text { Pre- and post-intervention delta: } 3.55 \\
\pm 5.03 \\
\text { Traditional therapy group } \\
\text { Pre- and post-intervention delta: } 3.45 \\
\pm 2.50\end{array}$ \\
\hline $\begin{array}{l}\text { Orsega-Smith } \\
\text { et al. } \\
2012 \\
\text { USA } \\
\text { [98] }\end{array}$ & $\begin{array}{l}\text { Elderly }(\mathrm{F} / \mathrm{M}, \\
\mathrm{N}=30 / 4) \\
\text { Age range: } 55-86 \mathrm{yrs} \\
\text { Mean age: } 72 \pm 8 \mathrm{yrs}\end{array}$ & $\begin{array}{l}\text { Intervention (No- } \\
\text { RCT) } \\
3 \text { groups: playing Wii } \\
\text { Fit for } 4 \text { wks vs. } \\
\text { playing Wii Fit for } 8 \\
\text { wks vs. passive } \\
\text { controls } \\
\text { Duration: } 4 \text { or } 8 \text { wks } \\
\text { (30 min, } 2 \\
\text { sessions/wk) } \\
\text { Location: Community } \\
\text { dwelling }\end{array}$ & $\begin{array}{l}\text { Yoga: Deep } \\
\text { Breathing, Half } \\
\text { Moon, Palm Tree; } \\
\text { aerobics: Hula Hoop; } \\
\text { balance: Balance } \\
\text { Bubble, Penguin } \\
\text { Slide, Snowboard } \\
\text { Slalom, Ski Jumping, } \\
\text { Ski Slalom, Table Tilt }\end{array}$ & $\begin{array}{l}\text { Balance (BBS), } \\
\text { mobility (8-feet TUG), } \\
\text { leg strength (STST), } \\
\text { balance confidence } \\
\text { (ABC, FES), } \\
\text { autonomy (ADL) }\end{array}$ & $\begin{array}{l}\text { Balance, and ability to complete } \\
\text { activities of daily living were improved } \\
\text { in the two Wii Fit groups. Leg strength } \\
\text { increased in the 4-wk intervention group } \\
\text { only, while balance confidence } \\
\text { increased in the } 8 \text {-wk intervention group } \\
\text { only. No change was noted in the } \\
\text { control group } \\
\text { Adverse events: No } \\
\text { ABC (no unit): } \\
\text { Wii Fit group (A: 4-week) } \\
\text { Pre- and post-intervention delta: } \mathbf{3 . 2 0} \\
\pm 13.88 \\
\text { Wii Fit group (B: 8-week) } \\
\text { Pre- and post-intervention delta: } \mathbf{6 . 0 7} \\
\pm 7.04 \\
\text { BBS (no unit): } \\
\text { Wii Fit group (A: 4-week) } \\
\text { Pre- and post-intervention delta: } \mathbf{1 . 4 4} \\
\pm 2.34 \\
\text { Wii Fit group (B: } 8 \text {-week) } \\
\text { Pre- and post-intervention delta: } \mathbf{1 . 2 2} \\
\pm 1.09\end{array}$ \\
\hline $\begin{array}{l}\text { Rendon et al. } \\
2012 \\
\text { USA } \\
{[9]}\end{array}$ & $\begin{array}{l}\text { Elderly }(F / M, \\
N=26 / 14 ; 6 \text { using an } \\
\text { assistive devise) } \\
\text { Age range: } 60-95 \mathrm{yrs} \\
\text { Mean age: } 85 \pm 5 \mathrm{yrs}\end{array}$ & $\begin{array}{l}\text { Intervention (RCT) } \\
2 \text { groups: Wii Fit vs. } \\
\text { passive control } \\
\text { Duration: } 6 \text { wks (35- } \\
45 \text { min, } 3 \\
\text { sessions/wk) } \\
\text { Location: Community } \\
\text { dwelling }\end{array}$ & $\begin{array}{l}\text { Strength: Lunge, } \\
\text { Single Leg Extension, } \\
\text { Single Leg Twist }\end{array}$ & $\begin{array}{l}\text { Primary: balance } \\
\text { (ABC, 8ft up and go } \\
\text { test) } \\
\text { Secondary: depression } \\
\text { (GDS) }\end{array}$ & $\begin{array}{l}\text { Wii Fit improved dynamic balance and } \\
\text { balance confidence. No effect on } \\
\text { depression score. } \\
\text { Adverse events: No }\end{array}$ \\
\hline
\end{tabular}




\begin{tabular}{|c|c|c|c|c|c|}
\hline $\begin{array}{l}\text { Toulotte et al. } \\
2012 \\
\text { France } \\
{[99]}\end{array}$ & $\begin{array}{l}\text { Elderly (some had an } \\
\text { history of falling; } \\
F / M, N=22 / 14 \text { ) } \\
\text { Age range: }>60 \text { yrs } \\
\text { Mean age: } 75 \pm 10 \text { yrs }\end{array}$ & $\begin{array}{l}\text { Intervention (RCT) } \\
4 \text { groups: passive } \\
\text { control vs. Wii-Fit, } \\
\text { adapted physical } \\
\text { activities vs. Wii Fit + } \\
\text { adapted physical } \\
\text { activities } \\
\text { Duration: } 20 \mathrm{wks}(60 \\
\text { min, } 1 \text { session/wk) } \\
\text { Location: Fitness } \\
\text { room }\end{array}$ & $\begin{array}{l}\text { Yoga activities and } \\
\text { some balance } \\
\text { activities (Soccer } \\
\text { Heading, Ski Jump, } \\
\text { Ski Slalom, Tightrope } \\
\text { Walk, and another } \\
\text { activity identified as } \\
\text { "game balls") }\end{array}$ & $\begin{array}{l}\text { Balance (static tests } \\
\text { only: a single leg } \\
\text { stance test }{ }^{\mathrm{a}} \text { and the Wii } \\
\text { Fit balance test; static } \\
\text { and dynamic test: } \\
\text { Tinetti test) }\end{array}$ & $\begin{array}{l}\text { Wii Fit significantly improved static } \\
\text { balance but not dynamic balance. The } \\
\text { conventional adapted PA training } \\
\text { improved both. Combining both } \\
\text { interventions did not induce additional } \\
\text { benefits. } \\
\text { Adverse events: No }\end{array}$ \\
\hline $\begin{array}{l}\text { Bieryla \& } \\
\text { Dold } \\
2013 \\
\text { USA } \\
{[7]}\end{array}$ & $\begin{array}{l}\text { Elderly }(F / M, \\
N=10 / 2) \\
\text { Age range: } 70-92 \mathrm{yrs} \\
\text { Mean age: } 82 \pm 6 \mathrm{yrs}\end{array}$ & $\begin{array}{l}\text { Intervention (RCT) } \\
2 \text { groups: Wii Fit vs. } \\
\text { passive control } \\
\text { Duration: } 3 \text { wks ( } 30 \\
\text { min, } 3 \text { sessions/wk) + } \\
\text { follow-up at } 4 \text {-wk } \\
\text { Location: Community } \\
\text { dwelling (supposedly: } \\
\text { "supervised") }\end{array}$ & $\begin{array}{l}\text { Yoga: } \text { Chair, Half } \\
\text { Moon, Warrior; } \\
\text { aerobics: Torso } \\
\text { Twists; balance: Ski } \\
\text { Jump Soccer, Heading }\end{array}$ & $\begin{array}{l}\text { Balance (BBS, TUG, } \\
\text { FAB, functional reach } \\
\text { test) and adherence } \\
\text { (retention rate) }\end{array}$ & $\begin{array}{l}\text { In the Wii Fit group, the retention rate } \\
\text { was } 4 / 6 \text { at the } 4 \text {-wk follow-up. The Wii } \\
\text { Fit training induced an improvement in } \\
\text { the BBS only. } \\
\text { Adverse events: No }\end{array}$ \\
\hline $\begin{array}{l}\text { Chao et al. } \\
2013 \\
\text { USA } \\
{[100]}\end{array}$ & $\begin{array}{l}\text { Assisted living } \\
\text { residents }(F / M, \\
N=5 / 2,3 \text { of them } \\
\text { having acquired } \\
\text { neurological } \\
\text { disorders) } \\
\text { Age range: } 80-94 \text { yrs } \\
\text { Mean age: } 86 \pm 5 \mathrm{yrs}\end{array}$ & $\begin{array}{l}\text { Intervention } \\
1 \text { group } \\
\text { Duration: } 8 \text { wks ( } 30 \\
\text { min, } 2 \text { sessions/wk) } \\
\text { Location: Assisted } \\
\text { living dwelling }\end{array}$ & $\begin{array}{l}\text { Yoga: Chair, Deep } \\
\text { Breathing; strength: } \\
\text { Lunge; aerobics: } \\
\text { Basic Run; balance: } \\
\text { Penguin Slide, Table } \\
\text { Tilt }\end{array}$ & $\begin{array}{l}\text { Balance \& mobility } \\
\text { (BBS, TUG, 6-min } \\
\text { walk test, FES), } \\
\text { perceived efficacy } \\
\text { (SSE, OEE), } \\
\text { acceptability } \\
\text { (questionnaire), safety }\end{array}$ & $\begin{array}{l}\text { The Wii Fit intervention was acceptable } \\
\text { and safe, and induced significant } \\
\text { improvements in BBS. Trends only ( } \mathrm{p}= \\
\text { 0.06) were noted for improvement in } \\
\text { other balance and mobility indexes. } \\
\text { Adverse events: No } \\
\text { BBS (no unit): } \\
\text { Wii Fit group } \\
\text { Pre-intervention: } \mathbf{4 0 . 9} \pm \mathbf{8 . 5} \\
\text { Post-intervention: } \mathbf{4 5 . 1} \pm \mathbf{8 . 3} \\
\text { TUG (sec): } \\
\text { Wii Fit group } \\
\text { Pre-intervention: } \mathbf{1 9 . 4} \pm \mathbf{5 . 5} \\
\text { Post-intervention: } \mathbf{1 5 . 8} \pm \mathbf{5 . 1}\end{array}$ \\
\hline $\begin{array}{l}\text { Janssen et al. } \\
2013 \\
\text { The } \\
\text { Netherland } \\
{[8]}\end{array}$ & $\begin{array}{l}\text { Home nursing } \\
\text { residents ( } F / M, \\
N=20 / 9 \text {, some had a } \\
\text { history of falling) } \\
\text { Average age: } 65-90\end{array}$ & $\begin{array}{l}\text { Intervention (No- } \\
\text { RCT) } \\
3 \text { groups: Wii Fit } \\
\text { (without history of } \\
\text { playing) vs. Wii Fit }\end{array}$ & $\begin{array}{l}\text { Table Tilt Plus } \\
\text { (Training Plus) and } 2 \\
\text { other games of } \\
\text { participants' choice }\end{array}$ & $\begin{array}{l}\text { Primary: balance } \\
\text { (BBS) } \\
\text { Secondary: physical } \\
\text { activity (LASAPAQ) }\end{array}$ & $\begin{array}{l}\text { No significant balance improvements in } \\
\text { either Wii Fit intervention groups. } \\
\text { However, subjects increased their } \\
\text { volume of physical activity by about } 60 \\
\text { min/day. }\end{array}$ \\
\hline
\end{tabular}




\begin{tabular}{|c|c|c|c|c|c|}
\hline & $\begin{array}{l}\text { yrs } \\
\text { Mean age: } 82 \pm 9 \text { yrs }\end{array}$ & $\begin{array}{l}\text { (with an history of } \\
\text { playing) vs. passive } \\
\text { control } \\
\text { Duration: } 12 \text { weeks } \\
\text { (10-15 min, } 2 \\
\text { sessions/wk) } \\
\text { Location: Assisted } \\
\text { living dwelling }\end{array}$ & & & Adverse events: No \\
\hline $\begin{array}{l}\text { Chao et al. } \\
2014 \\
\text { USA } \\
{[101]}\end{array}$ & $\begin{array}{l}\text { Assisted living } \\
\text { resident } \\
(F / M, N=24 / 8) \\
\text { Mean age: } 85 \pm 6 \mathrm{yrs}\end{array}$ & $\begin{array}{l}\text { Intervention (RCT) } \\
2 \text { groups: Wii Fit vs. } \\
\text { "education" semi- } \\
\text { passive control } \\
\text { Duration: } 4 \text { wks ( } 30 \\
\text { min, } 2 \text { sessions/wk) } \\
\text { Location: Assisted } \\
\text { living dwelling }\end{array}$ & $\begin{array}{l}\text { Yoga: Chair, Deep } \\
\text { Breathing; strength: } \\
\text { Lunge; balance: } \\
\text { Penguin Slide, Table } \\
\text { Tilt; aerobics: Basic } \\
\text { Run }\end{array}$ & $\begin{array}{l}\text { Balance and physical } \\
\text { function (BBS, TUG, } \\
\text { 6-min walk test, FES, } \\
\text { SEE), depression } \\
\text { (GDS), quality of life } \\
\text { (SF-8) }\end{array}$ & $\begin{array}{l}\text { Significant improvements in balance } \\
\text { related-function and depression } \\
\text { parameters were found in the Wii Fit } \\
\text { group only. Wii Fit might be considered } \\
\text { as a potential activity for older adults in } \\
\text { assisted living dwellings. } \\
\text { Adverse events: No } \\
\text { BBS (no unit): } \\
\text { Wii Fit group } \\
\text { Pre-intervention: } \mathbf{4 0 . 5 3} \pm \mathbf{6 . 5 9} \\
\text { Post-intervention: } \mathbf{4 3 . 9 3} \pm \mathbf{6 . 3 4} \\
\text { TUG (sec): } \\
\text { Wii Fit group } \\
\text { Pre-intervention: } \mathbf{1 8 . 5 2} \pm \mathbf{5 . 6 0} \\
\text { Post-intervention: } \mathbf{1 5 . 2 7} \pm \mathbf{4 . 6 8}\end{array}$ \\
\hline $\begin{array}{l}\text { Cho et al. } \\
2014 \\
\text { Korea } \\
{[102]}\end{array}$ & $\begin{array}{l}\text { Elderly }(N=32) \\
\text { Mean age: } 78 \pm 1 \text { yrs }\end{array}$ & $\begin{array}{l}\text { Intervention (RCT) } \\
2 \text { groups: Wii Fit vs. } \\
\text { passive control } \\
\text { Duration: } 8 \text { wks ( } 30 \\
\text { min, } 3 \text { sessions/wk) } \\
\text { Location: not } \\
\text { specified }\end{array}$ & $\begin{array}{l}\text { Balance: Balance } \\
\text { Bubble, Ski Slalom, } \\
\text { Table Tilt }\end{array}$ & $\begin{array}{l}\text { Balance (Romberg } \\
\text { test) }\end{array}$ & $\begin{array}{l}\text { Significant improvements were noted in } \\
\text { the Wii Fit group only. } \\
\text { Adverse events: No }\end{array}$ \\
\hline $\begin{array}{l}\text { Nicholson et } \\
\text { al. } \\
2015 \\
\text { Australia } \\
{[105]}\end{array}$ & $\begin{array}{l}\text { Elderly }(F / M, \\
N=14 / 27) \\
\text { Age range: } 65-84 \mathrm{yrs} \\
\text { Mean age: } 75 \pm 5 \mathrm{yrs}\end{array}$ & $\begin{array}{l}\text { Intervention (RCT) } \\
2 \text { groups: Wii Fit vs. } \\
\text { usual exercise } \\
\text { Duration: } 6 \text { wks ( } 30 \\
\text { min, } 3 \text { sessions /wk) } \\
\text { Location: Community } \\
\text { dwelling }\end{array}$ & $\begin{array}{l}\text { Balance: Penguin } \\
\text { Slide, Ski Jump, Ski } \\
\text { Slalom, Soccer } \\
\text { Heading, Table Tilt, } \\
\text { Tightrope Walk; } \\
\text { training plus: } \\
\text { Snowball Fight, } \\
\text { Perfect } 10\end{array}$ & $\begin{array}{l}\text { Balance and mobility } \\
\text { (TUG, functional } \\
\text { reach tests, single leg } \\
{\text { stance test }{ }^{a} \text {, STST, }} \\
\text { Icon-typed FES, } \\
\text { walking speed), } \\
\text { enjoyment (PACES), } \\
\text { adherence (playing } \\
\text { frequency) }\end{array}$ & $\begin{array}{l}\text { The Wii Fit group showed more } \\
\text { improvement compared to the control } \\
\text { group for the followings: TUG, lateral } \\
\text { reach, left-leg single leg stance test and } \\
\text { gait speed. Interestingly, enjoyment } \\
\text { increased during the intervention and } \\
\text { the adherence was very high (average } \\
\text { attendance: } 17.5 \text { out of } 18 \\
\text { recommended sessions) }\end{array}$ \\
\hline
\end{tabular}




\begin{tabular}{|c|c|c|c|c|c|}
\hline & & & & & $\begin{array}{l}\text { Adverse events: exacerbations of lower } \\
\text { back pain }(\mathrm{N}=2) \\
\text { TUG (sec): } \\
\text { Wii Fit group } \\
\text { Pre- and post-intervention delta: - } \\
\text { 0.61 } \pm 0.79 \\
\text { Traditional therapy group } \\
\text { Pre- and post-intervention delta: - } \\
0.14 \pm 0.88\end{array}$ \\
\hline $\begin{array}{l}\text { Roopchand- } \\
\text { Martin et al. } \\
2015 \\
\text { Jamaica } \\
{[106]}\end{array}$ & $\begin{array}{l}\text { Elderly }(F / M, \\
N=26 / 7) \\
\text { Mean age: } 70 \pm 7 \mathrm{yrs}\end{array}$ & $\begin{array}{l}\text { Intervention } \\
1 \text { group } \\
\text { Duration: } 6 \text { wks ( } 30 \\
\text { min, } 2 \text { sessions/wk) } \\
\text { Location: Community } \\
\text { dwelling }\end{array}$ & $\begin{array}{l}\text { Yoga: Tree; balance: } \\
\text { Balance Bubble, } \\
\text { Penguin Slide, } \\
\text { Snowboard Slalom, } \\
\text { Soccer Heading, } \\
\text { Table Tilt; training } \\
\text { plus: Obstacle } \\
\text { Course, Skateboard }\end{array}$ & $\begin{array}{l}\text { Balance and function } \\
\text { (BBS, MCTSIB, } \\
\text { MDRT, SEBT) }\end{array}$ & $\begin{array}{l}\text { Significant balance and functional } \\
\text { improvements were noted at the end of } \\
\text { the Wii Fit intervention (i.e. for BBS, } \\
\text { MDRT, SEBT, but not for MCTSIB) } \\
\text { Adverse events: No } \\
\text { BBS (no unit): } \\
\text { Wii Fit group } \\
\text { Pre- and post-intervention delta: } \mathbf{1 . 5 4} \\
\pm \mathbf{2 . 6 0}\end{array}$ \\
\hline \multicolumn{6}{|c|}{ Seniors presenting balance impairments } \\
\hline $\begin{array}{l}\text { Williams et al. } \\
2010 \\
\text { UK } \\
{[108]}\end{array}$ & $\begin{array}{l}\text { Elderly with an } \\
\text { history of falling } \\
(N=21) \\
\text { Mean age: } 77 \pm 5 \text { yrs }\end{array}$ & $\begin{array}{l}\text { Intervention (RCT) } \\
2 \text { groups: Wii Fit vs. } \\
\text { standard care } \\
\text { Duration: } 12 \text { wks ( } 2 \\
\text { sessions/wk) } \\
\text { Location: } \\
\text { Rehabilitation center }\end{array}$ & $\begin{array}{l}\text { Yoga: Deep } \\
\text { Breathing; aerobics: } \\
\text { Basic Step, Hula } \\
\text { Hoop, Running } \\
\text { activities; Balance: } \\
\text { Ski Jump, Ski Slalom, } \\
\text { Soccer Heading, } \\
\text { Table Tilt, }\end{array}$ & $\begin{array}{l}\text { Functional balance } \\
\text { (BBS, Tinetti test), } \\
\text { static balance (Wii Fit } \\
\text { age test) Balance } \\
\text { confidence (FES), } \\
\text { acceptability } \\
\text { (retention, playing } \\
\text { frequency, interviews) }\end{array}$ & $\begin{array}{l}\text { The intervention met a high rate of } \\
\text { acceptability. Balance improved in the } \\
\text { Wii Fit group only. } \\
\text { Adverse events: fall ( } \mathrm{N}=1 \text {, no injury) } \\
\text { BBS (no unit): } \\
\text { Wii Fit group } \\
\text { Pre-intervention: } 43.7 \pm 9.5 \\
\text { Post-intervention: } \mathbf{4 4 . 8} \pm \mathbf{1 1 . 8} \\
\text { Traditional therapy group } \\
\text { Pre-intervention: } 36.3 \pm 9.9 \\
\text { Post-intervention: } 39.0 \pm \mathbf{1 0 . 2}\end{array}$ \\
\hline $\begin{array}{l}\text { Agmon et al. } \\
2011 \\
\text { USA } \\
{[109]}\end{array}$ & $\begin{array}{l}\text { Elderly with balance } \\
\text { impairment }(F / M \text {, } \\
N=4 / 3) \\
\text { Age range: } 78-92 \text { yrs } \\
\text { Mean age: } 84 \pm 5 \text { yrs }\end{array}$ & $\begin{array}{l}\text { Intervention } \\
1 \text { group } \\
\text { Duration: } 13 \text { wks ( } 30 \\
\text { min, } 3 \text { sessions/wk) } \\
\text { Location: Home }\end{array}$ & $\begin{array}{l}\text { Balance: Basic Step, } \\
\text { Ski Slalom, Soccer } \\
\text { Heading, Table Tilt }\end{array}$ & $\begin{array}{l}\text { Primary: Balance } \\
\text { (BBS) } \\
\text { Secondary: mobility } \\
\text { (4-meter walk test), } \\
\text { enjoyment (PACES), } \\
\text { feasibility (playing } \\
\text { time), safety }\end{array}$ & $\begin{array}{l}\text { The Wii Fit intervention increased } \\
\text { balance and mobility. Some activities } \\
\text { were more enjoyable than the others. } \\
\text { Adherence was associated with } \\
\text { enjoyment. } \\
\text { Adverse events: hip and neck strain } \\
\text { ( } \mathrm{N}=1 \text { and } 2 \text {, respectively) }\end{array}$ \\
\hline
\end{tabular}




\begin{tabular}{|c|c|c|c|c|c|}
\hline & & & & (interviews) & $\begin{array}{l}\text { BBS (no unit): } \\
\text { Wii Fit group } \\
\text { Pre-intervention: } 49.0 \pm 2.1 \\
\text { Post-intervention: } 53.0 \pm 1.8\end{array}$ \\
\hline $\begin{array}{l}\text { Bainbridge et } \\
\text { al. } \\
2011 \\
\text { USA } \\
{[10]}\end{array}$ & $\begin{array}{l}\text { Elderly with } \\
\text { perceived balance } \\
\text { deficit }(F / M, N=7 / 1) \\
\text { Age range: } 65-87 \text { yrs } \\
\text { Mean age: } 75 \pm 8 \text { yrs }\end{array}$ & $\begin{array}{l}\text { Intervention } \\
1 \text { group } \\
\text { Duration: } 6 \text { wks ( } 30 \\
\text { min, } 2 \text { sessions/wk) } \\
\text { Location: Community } \\
\text { dwelling }\end{array}$ & $\begin{array}{l}\text { Yoga: Half Moon, } \\
\text { Warrior; Balance: } \\
\text { Penguin Slide, Ski } \\
\text { Jump, Ski Slalom, } \\
\text { Soccer Heading, } \\
\text { Table Title, Tightrope } \\
\text { Walk }\end{array}$ & $\begin{array}{l}\text { Balance (BBS, ABC, } \\
\text { MDRT), COP } \\
\text { excursion } \\
\text { measurements) and } \\
\text { other parameters } \\
\text { (ankle range of motion } \\
\text { tests...) }\end{array}$ & $\begin{array}{l}\text { No statistically significant changes, but } \\
4 \text { patients (over the } 6 \text { who finished the } \\
\text { intervention) demonstrated } \\
\text { improvements on the BBS, based on } \\
\text { established clinical guidelines. } \\
\text { Adverse events: No }\end{array}$ \\
\hline $\begin{array}{l}\text { Jorgensen et } \\
\text { al. } \\
2012 \\
\text { Denmark } \\
{[111]}\end{array}$ & $\begin{array}{l}\text { Elderly with } \\
\text { perceived balance } \\
\text { deficit }(F / M \text {, } \\
N=40 / 18) \\
\text { Mean age: } 75 \pm 6 \text { yrs }\end{array}$ & $\begin{array}{l}\text { Intervention (RCT) } \\
2 \text { groups: Wii Fit vs. } \\
\text { passive control } \\
\text { Duration: } 10 \text { wks }(35 \\
\text { min, } 2 \text { sessions/wk) } \\
\text { Location: Community } \\
\text { dwelling }\end{array}$ & $\begin{array}{l}\text { Balance: Penguin } \\
\text { Slide, Ski Slalom, } \\
\text { Table Tilt, Tightrope } \\
\text { Walk; training plus: } \\
\text { Perfect } 10\end{array}$ & $\begin{array}{l}\text { Primary: strength } \\
\text { (maximal voluntary } \\
\text { contraction of leg } \\
\text { extensors (MVC), } \\
\text { Rate of Force } \\
\text { Development (RFD), } \\
\text { static balance (COP } \\
\text { velocity moment) } \\
\text { Secondary: mobility } \\
\text { (TUG, STST), balance } \\
\text { confidence (FES), } \\
\text { motivation } \\
\text { (questionnaire) }\end{array}$ & $\begin{array}{l}\text { Compared to controls, the Wii Fit group } \\
\text { exhibited increased strength after } 10 \\
\text { weeks of training. Mobility and balance } \\
\text { confidence parameters also showed an } \\
\text { improvement in the Wii Fit group only. } \\
\text { Motivation for Wii Fit training was } \\
\text { found to be high. } \\
\text { Adverse events: No } \\
\text { TUG (sec): } \\
\text { Wii Fit group } \\
\text { Pre-intervention: } \mathbf{1 0 . 3} \pm \mathbf{3 . 8} \\
\text { Post-intervention: } \mathbf{9 . 0} \pm \mathbf{3 . 2}\end{array}$ \\
\hline \multicolumn{6}{|c|}{ Seniors with acquired neurological alterations } \\
\hline $\begin{array}{l}\text { dos Santos et } \\
\text { al. } \\
2012 \\
\text { Brazil } \\
{[114]}\end{array}$ & $\begin{array}{l}\text { Patients with } \\
\text { Parkinson's disease } \\
\text { (stages } 1 \text { and } 2 \text { on } \\
\text { Hoehn \& Yahr scale, } \\
N=16 \text { ) } \\
\text { Mean age: } 69 \pm 8 \text { yrs }\end{array}$ & $\begin{array}{l}\text { Intervention } \\
2 \text { groups: patients } v s \text {. } \\
\text { healthy controls, } \\
N=11) \\
\text { Duration: } 7 \text { wks ( } 20- \\
30 \text { min, } 2 \\
\text { sessions/wk) + follow- } \\
\text { up after } 2 \text { months } \\
\text { Location: } \\
\text { Rehabilitation center } \\
\text { (supposedly: } \\
\text { «Overseen by a } \\
\text { physiotherapist») }\end{array}$ & $\begin{array}{l}\text { Strength: Single Leg } \\
\text { Extension, Torso } \\
\text { Twists; aerobics: } \\
\text { Basic Step; balance: } \\
\text { Penguin Slide, Soccer } \\
\text { Heading, Table Tilt; } \\
\text { training plus: Basic } \\
\text { Run Plus, Obstacle } \\
\text { Course, Rhythm } \\
\text { Parade, Tilt City }\end{array}$ & $\begin{array}{l}\text { Stability (functional } \\
\text { reach test) and motor } \\
\text { learning (score } \\
\text { performed in the } \\
\text { selected games before } \\
\text { and after the } \\
\text { intervention) }\end{array}$ & $\begin{array}{l}7 \text { of the } 10 \text { tested games induced the } \\
\text { same learning in Parkinson's disease } \\
\text { patients compared with healthy subjects. } \\
\text { These patients were also able to transfer } \\
\text { and retained ( }+2 \text {-mths follow-up) their } \\
\text { learning on a similar but untrained } \\
\text { functional task. } \\
\text { Adverse events: No }\end{array}$ \\
\hline Esculier et al. $^{\mathrm{c}}$ & Patients with & Intervention & Yoga: Deep & Functional balance & Improvements in every outcome (except \\
\hline
\end{tabular}




\begin{tabular}{|c|c|c|c|c|c|}
\hline $\begin{array}{l}2012 \\
\text { Canada } \\
{[113]}\end{array}$ & $\begin{array}{l}\text { Parkinson's disease } \\
(F / M, N=5 / 6) \\
\text { Age range: } 48-80 \mathrm{yrs} \\
\text { Mean age: } 62 \pm 11 \mathrm{yrs}\end{array}$ & $\begin{array}{l}2 \text { groups: patients } v s . \\
\text { healthy controls, } F / M \text {, } \\
N=4 / 5) \\
\text { Duration: } 6 \text { wks ( } 40 \\
\text { min, } 3 \text { sessions } / \text { wk) } \\
\text { Location: Home }\end{array}$ & $\begin{array}{l}\text { Breathing; aerobics: } \\
\text { Hula-Hoop; balance: } \\
\text { Balance Bubble, } \\
\text { Penguin Slide, Ski } \\
\text { Jump, Ski Slalom, } \\
\text { Table Tilt }\end{array}$ & $\begin{array}{l}\text { and mobility (ABC, } \\
\text { STST, TUG, Tinetti } \\
\text { test, 10-m walk test, } \\
\text { CBM), static balance } \\
\text { (single leg stance test }{ }^{\mathrm{a}} \text {, } \\
\text { COP excursion) }\end{array}$ & $\begin{array}{l}\text { for } \mathrm{ABC} \text { ) in the two groups. A home- } \\
\text { based Wii Fit improved static and } \\
\text { dynamic balance, mobility and } \\
\text { functional abilities of people affected by } \\
\text { Parkinson's disease } \\
\text { Adverse events: No }\end{array}$ \\
\hline $\begin{array}{l}\text { Pompeu et al. } \\
2012 \\
\text { Brazil } \\
{[116]}\end{array}$ & $\begin{array}{l}\text { Patients with } \\
\text { Parkinson's disease } \\
\text { (stages } 1 \text { and } 2 \text { on } \\
\text { Hoehn \& Yahr scale, } \\
F / M, N=15 / 17 \text { ) } \\
\text { Age range: } 60-85 \mathrm{yrs} \\
\text { Mean age: } 67 \pm 8 \text { yrs }\end{array}$ & $\begin{array}{l}\text { Intervention (RCT) } \\
2 \text { groups: Wii Fit vs. } \\
\text { traditional balance } \\
\text { training } \\
\text { Duration: } 7 \text { wks }(30 \\
\text { min, } 2 \text { sessions/wk) + } \\
\text { follow-up } 60 \text { days } \\
\text { after } \\
\text { Location: } \\
\text { Rehabilitation center }\end{array}$ & $\begin{array}{l}\text { Strength: Single Leg } \\
\text { Extension, Torso } \\
\text { Twist; aerobics: Basic } \\
\text { Step, Basic Run; } \\
\text { balance: Penguin } \\
\text { Slide, Soccer } \\
\text { Heading, Table Tilt; } \\
\text { training plus: } \\
\text { Obstacle Course, } \\
\text { Rhythm Parade, Tilt } \\
\text { City }\end{array}$ & $\begin{array}{l}\text { Primary: performance } \\
\text { in daily activities } \\
\text { Secondary: balance } \\
\text { (static: single leg } \\
\text { stance test }{ }^{\mathrm{a}} \text {, dynamic: } \\
\text { BBS), cognition } \\
\text { (Montreal cognitive } \\
\text { assessment) }\end{array}$ & $\begin{array}{l}\text { Same improvements in Wii Fit and } \\
\text { traditional balance training groups } \\
\text { (maintained at } 60 \text { days follow-up). No } \\
\text { additional advantage for the Wii Fit } \\
\text { group. } \\
\text { Adverse events: No } \\
\\
\frac{\text { BBS (no unit) }}{\text { Wii Fit group }} \\
\text { Pre- and post-intervention delta: } \mathbf{1 . 4} \\
\pm \mathbf{2 . 6} \\
\text { Traditional therapy group } \\
\text { Pre- and post-intervention delta: } \mathbf{1 . 1} \\
\pm \mathbf{2 . 1}\end{array}$ \\
\hline $\begin{array}{l}\text { Padala et al. } \\
2012 \\
\text { USA } \\
{[115]}\end{array}$ & $\begin{array}{l}\text { Patients with an } \\
\text { history of mild } \\
\text { Alzheimer's Dementia } \\
\text { (F/M, } N=16 / 6) \\
\text { Mean age: } 80 \pm 7 \text { yrs }\end{array}$ & $\begin{array}{l}\text { Intervention (RCT) } \\
2 \text { groups: Wii Fit vs. } \\
\text { walking } \\
\text { Duration: } 8 \text { wks ( } 30 \\
\text { min, } 5 \text { sessions/wk) } \\
\text { Location: Assisted } \\
\text { living center }\end{array}$ & $\begin{array}{l}\text { Yoga: Chair, Half } \\
\text { moon, Sun Salutation } \\
\text { Warrior; strength: } \\
\text { Lunge, Single Leg } \\
\text { Extension, Torso } \\
\text { Twist; balance: } \\
\text { Balance Bubble, } \\
\text { Penguin Slide, Ski } \\
\text { Jump, Ski Slalom, } \\
\text { Soccer Heading, } \\
\text { Table Tilt }\end{array}$ & $\begin{array}{l}\text { Primary: balance } \\
\text { (BBS, TUG, Tinetti } \\
\text { test) } \\
\text { Secondary: functional } \\
\text { ability (ADL \& } \\
\text { instrumental ADL), } \\
\text { quality of life (quality } \\
\text { of life in Alzheimer's } \\
\text { disease scale), } \\
\text { cognition (mini mental } \\
\text { state examination) }\end{array}$ & $\begin{array}{l}\text { Significant improvements for balance } \\
\text { outcomes in the Wii Fit group only } \\
\text { (trends for the walking group). No } \\
\text { significant changes in other outcomes, } \\
\text { except for quality of life (walking group } \\
\text { only). } \\
\text { Adverse events: No } \\
\text { BBS (no unit) } \\
\text { Wii Fit group } \\
\text { Pre-intervention: } 43.48 .9 \\
\text { Post-intervention: } 47.55 .9 \\
\text { Traditional therapy group } \\
\text { Pre-intervention: } 41.3 \pm 7.6 \\
\text { Post-intervention: } 46.9 \pm 6.3 \\
\text { TUG (sec) } \\
\text { Wii Fit group } \\
\text { Pre-intervention: } 14.7 \pm 7.2 \\
\text { Post-intervention: } \mathbf{1 4 . 3} \pm \mathbf{6 . 8} \\
\text { Traditional therapy group }\end{array}$ \\
\hline
\end{tabular}




\begin{tabular}{|c|c|c|c|c|c|}
\hline & & & & & $\begin{array}{l}\text { Pre-intervention: } 14.9 \pm 4.7 \\
\text { Post-intervention: } 12.8 \pm 3.2\end{array}$ \\
\hline $\begin{array}{l}\text { Barcala et al. } \\
2013 \\
\text { Brazil } \\
{[17]}\end{array}$ & $\begin{array}{l}\text { Hemiplegic stroke } \\
\text { patients }(F / M, \\
N=11 / 9) \\
\text { Age range: } \\
\text { Mean age: } 64 \pm 14 \mathrm{yrs}\end{array}$ & $\begin{array}{l}\text { Intervention (RCT) } \\
2 \text { groups: } \\
\text { conventional therapy } \\
+ \text { Wii Fit vs. } \\
\text { conventional therapy } \\
\text { + balance training } \\
\text { Duration: } 5 \mathrm{wks}(30 \\
\text { min, } 2 \text { sessions } / \mathrm{wk} \text { ) } \\
\text { Location: } \\
\text { Rehabilitation center }\end{array}$ & $\begin{array}{l}\text { Balance: Penguin } \\
\text { Slide, Table Tilt, } \\
\text { Tightrope Walk }\end{array}$ & $\begin{array}{l}\text { Functional balance } \\
\text { (BBS), static balance } \\
\text { (stabilometry), } \\
\text { functional mobility, } \\
\text { independence (TUG, } \\
\text { functional } \\
\text { independence test) }\end{array}$ & $\begin{array}{l}\text { Both groups showed significant } \\
\text { improvements in all parameters. No } \\
\text { statistical differences were noted } \\
\text { between the two groups emphasizing } \\
\text { the efficacy of the Wii Fit therapy for } \\
\text { functional recovery in hemiplegic stroke } \\
\text { patient. } \\
\text { Adverse events: No } \\
\\
\text { BBS (no unit) } \\
\text { Wii Fit group } \\
\text { Pre-intervention: } \mathbf{3 9 . 6} \pm \mathbf{6 . 4 3} \\
\text { Post-intervention: } \mathbf{4 1 . 9} \pm \mathbf{6 . 9 1} \\
\text { Traditional therapy group } \\
\text { Pre-intervention: } \mathbf{3 7 . 2} \pm \mathbf{5 . 2 2} \\
\text { Post-intervention: } \mathbf{4 2 . 2} \pm \mathbf{4 . 8} \\
\text { TUG (sec) } \\
\text { Wii Fit group } \\
\text { Pre-intervention: } 27.9 \pm \mathbf{8 . 2 2} \\
\text { Post-intervention: } \mathbf{2 4 . 3} \pm \mathbf{8 . 6 4} \\
\text { Traditional therapy group } \\
\text { Pre-intervention: } 28.1 \pm \mathbf{3 . 1 0} \\
\text { Post-intervention: } 25.2 \pm \mathbf{2 . 7 8}\end{array}$ \\
\hline $\begin{array}{l}\text { Mhatre et al. } \\
2013 \\
\text { USA } \\
{[117]}\end{array}$ & $\begin{array}{l}\text { Patients with } \\
\text { Parkinson's disease } \\
\text { (stages } 2.5 \text { or } 3 \text { on } \\
\text { Hoehn \& Yahr scale, } \\
F / M, N=6 / 4 \text { ) } \\
\text { Age range: } 44-91 \text { yrs } \\
\text { Mean age: } 67 \text { yrs }\end{array}$ & $\begin{array}{l}\text { Intervention } \\
1 \text { group } \\
\text { Duration: } 8 \text { wks ( } 30 \\
\text { min, } 3 \text { sessions/wk) } \\
\text { Location: } \\
\text { Rehabilitation center }\end{array}$ & $\begin{array}{l}\text { Balance: "marble } \\
\text { tracking", "skiing", } \\
\text { "bubble rafting" } \\
\text { (possibly: Table Tilt, } \\
\text { Ski Slalom and } \\
\text { Balance Bubble) }\end{array}$ & $\begin{array}{l}\text { Primary: Balance } \\
\text { (BBS; DGI; } \\
\text { Sharpened Romberg; } \\
\text { Wii Balance Board- } \\
\text { assisted postural sway } \\
\text { tests) } \\
\text { Secondary: Balance } \\
\text { (ABC) and depression } \\
\text { (GDS) }\end{array}$ & $\begin{array}{l}\text { Significant improvements in BBS (3.3) } \\
\text { and some other balance \& gait } \\
\text { outcomes, but not in balance confidence } \\
\text { (ABC) or mood (GDS). } \\
\text { Adverse events: No } \\
\text { ABC (no unit) } \\
\text { Wii Fit group } \\
\text { Pre-intervention: } \mathbf{8 3 . 5} \pm \mathbf{5 . 3} \\
\text { Post-intervention: } 82.5 \pm \mathbf{3 . 6} \\
\text { BBS (no unit) } \\
\text { Wii Fit group } \\
\text { Pre-intervention: } \mathbf{4 8 . 8} \pm \mathbf{3 . 2} \\
\text { Post-intervention: } \mathbf{5 2 . 1} \pm \mathbf{2 . 3}\end{array}$ \\
\hline Bower et al. & Stroke inpatients & Intervention (RCT) & A selection of 18 & Primary: adherence & The recruitment rate (21\%), eligibility \\
\hline
\end{tabular}




\begin{tabular}{|c|c|c|c|c|c|}
\hline $\begin{array}{l}2014 \\
\text { Australia } \\
{[89]}\end{array}$ & $\begin{array}{l}(F / M, N=13 / 17) \\
\text { Mean age: } 64 \pm 15 \mathrm{yrs}\end{array}$ & $\begin{array}{l}2 \text { groups: Wii Fit } \\
\text { balance training vs. } \\
\text { Wii Sports upper limb } \\
\text { training } \\
\text { Duration: } 2-4 \text { weeks } \\
\text { (45 min, } 3 \\
\text { sessions/wk) } \\
\text { Location: } \\
\text { Rehabilitation center }\end{array}$ & $\begin{array}{l}\text { activities among the } \\
66 \text { activities proposed } \\
\text { in the Wii Fit Plus } \\
\text { software (including } \\
\text { Deep Breathing, Ski } \\
\text { Slalom, Basic Run } \\
\text { and others...) }\end{array}$ & $\begin{array}{l}\text { (retention, attendance, } \\
\text { playing time), } \\
\text { acceptability } \\
\text { (recruitment rate, } \\
\text { questionnaire), safety } \\
\text { (questionnaire) } \\
\text { Secondary: Balance } \\
\text { (Step Test, } \text { Wii } \\
\text { Balance Board Test), } \\
\text { Functional autonomy } \\
\text { (functional reach test, } \\
\text { upper limb - motor } \\
\text { assessment scale), } \\
\text { mobility (TUG, } \\
\text { STREAM), balance } \\
\text { confidence (FES), }\end{array}$ & $\begin{array}{l}\text { rate }(86 \%) \text {, retention rate }(90 \text { and } 70 \% \\
\text { respectively at } 2 \text { and } 4 \text { wks) and } \\
\text { adherence rate }(99 \text { and } 87 \%) \text { indicated } \\
\text { that a Wii Fit intervention would be } \\
\text { feasible in stroke inpatients. All the } \\
\text { patients enjoyed the intervention, which } \\
\text { was described as safe. However, trends } \\
\text { only were noted for improvements in } \\
\text { some of the balance tests. } \\
\text { Adverse events: Falls (N=4), no } \\
\text { subsequent injury } \\
\text { TUG (sec): } \\
\text { Wii Fit group } \\
\text { Pre- and post-intervention delta: - } \\
\mathbf{1 1 . 2} \pm \mathbf{1 0 . 3}\end{array}$ \\
\hline $\begin{array}{l}\text { Esculier et al. }{ }^{c} \\
2014 \\
\text { Canada } \\
{[118]}\end{array}$ & $\begin{array}{l}\text { Patients with } \\
\text { Parkinson's disease } \\
\text { (stages } 3.5 \text { or more on } \\
\text { Hoehn \& Yahr scale, } \\
F / M, N=3 / 5 \text { ) } \\
\text { Mean age: } 64 \pm 12 \text { yrs }\end{array}$ & $\begin{array}{l}\text { Intervention } \\
2 \text { groups: patients } v s . \\
\text { healthy controls }(F / M \text {, } \\
N=3 / 5) \\
\text { Duration: } 6 \mathrm{wks}(40 \\
\text { min, } 3 \text { sessions/wk) } \\
\text { Location: Home }\end{array}$ & $\begin{array}{l}\text { A selection of balance } \\
\text { and strength activities } \\
\text { involving lower limb } \\
\text { muscles (i.e. using } \\
\text { semi-squats } \\
\text { Positions) }\end{array}$ & $\begin{array}{l}\text { Lower limb } \\
\text { corticomotor } \\
\text { excitability } \\
\text { (transcranial magnetic } \\
\text { stimulation) }\end{array}$ & $\begin{array}{l}\text { Wii Fit training improved lower limb } \\
\text { corticomotor excitability in Parkinson's } \\
\text { patients. Depending on the experimental } \\
\text { conditions, these improvements were } \\
\text { similar or more important when } \\
\text { compared to healthy subjects. Home- } \\
\text { based interventions including visual } \\
\text { feedbacks could be beneficial for } \\
\text { functional improvement in Parkinson's } \\
\text { patients. } \\
\text { Adverse events: No }\end{array}$ \\
\hline $\begin{array}{l}\text { Gonçalves et } \\
\text { al. } \\
2014 \\
\text { Brazil } \\
{[119]}\end{array}$ & $\begin{array}{l}\text { Patients with } \\
\text { Parkinson's disease } \\
\text { (stages } 2 \text { to } 4 \text { on } \\
\text { Hoehn \& Yahr scale, } \\
F / M, N=8 / 7 \text { ) } \\
\text { Mean age: } 69 \pm 10 \text { yrs }\end{array}$ & $\begin{array}{l}\text { Intervention } \\
\text { 1 group } \\
\text { Duration: } 7 \text { weeks ( } 40 \\
\text { min, } 2 \text { sessions/wk) } \\
\text { Location: Hospital } \\
\text { (supposedly, not } \\
\text { specified) }\end{array}$ & $\begin{array}{l}\text { Balance: Ski Jump, } \\
\text { Ski Slalom, Soccer } \\
\text { Header; } \\
\text { aerobics: Free Step, } \\
\text { Rhythm Boxing; } \\
\text { training plus: Island } \\
\text { Cycling, Rhythm } \\
\text { Parade (supposedly), } \\
\text { Segway Circuit } \\
\text { (+2 other activities } \\
\text { that were not } \\
\text { explicitly named) }\end{array}$ & $\begin{array}{l}\text { Functional mobility } \\
\text { (UPDRS, SE, FIM), } \\
\text { gait (number of steps, } \\
\text { walking speed) }\end{array}$ & $\begin{array}{l}\text { The Wii Fit program induced gait } \\
\text { improvement, but statistical significance } \\
\text { was not indicated. Functional mobility } \\
\text { was significantly improved (i.e. } \\
\text { decrease in UPDRS score, and increase } \\
\text { in SE and FIM scores) } \\
\text { Adverse events: No }\end{array}$ \\
\hline Hung et al. & Chronic stroke & Intervention (RCT) & Yoga: Warrior; & Balance (a series of & At the end of the intervention, Wii Fit \\
\hline
\end{tabular}




\begin{tabular}{|c|c|c|c|c|c|}
\hline $\begin{array}{l}2014 \\
\text { China } \\
{[90]}\end{array}$ & $\begin{array}{l}\text { patients } \\
(F / M, N=10 / 18) \\
\text { Mean age: } 54 \pm 10 \mathrm{yrs}\end{array}$ & $\begin{array}{l}2 \text { groups: Wii Fit vs. } \\
\text { traditional weight- } \\
\text { shift training } \\
\text { Duration: } 12 \mathrm{wks}(30 \\
\text { min, } 2 \text { sessions/wk) }+ \\
\text { follow-up at } 3 \text { months } \\
\text { Location: } \\
\text { rehabilitation center } \\
\text { (supposedly: } \\
\text { according to pictures, } \\
\text { "supervised by an } \\
\text { occupational } \\
\text { therapist") }\end{array}$ & $\begin{array}{l}\text { balance: Balance } \\
\text { Bubble, Penguin } \\
\text { Slide, Ski Slalom, } \\
\text { Soccer Heading, } \\
\text { Table Tilt; aerobics: } \\
\text { Basic Step }\end{array}$ & $\begin{array}{l}\text { COP excursion tests, } \\
\text { FES), function } \\
\text { (forward reach, TUG), } \\
\text { enjoyment (PACES) }\end{array}$ & $\begin{array}{l}\text { induced a higher increase in some COP } \\
\text { excursion tests compared to the } \\
\text { traditional weigh-shift training group. } \\
\text { However, at a 3-month follow-up, these } \\
\text { effects were not maintained, while the } \\
\text { traditional weight-shift group showed } \\
\text { higher improvements. Both types of } \\
\text { intervention showed significant } \\
\text { improvements in balance and functional } \\
\text { outcomes, and the enjoyment was } \\
\text { higher in the Wii Fit group. } \\
\text { Adverse events: No } \\
\text { TUG (sec): } \\
\text { Wii Fit group } \\
\text { Pre-intervention: } \mathbf{2 6 . 0 6} \pm \mathbf{1 2 . 0 5} \\
\text { Post-intervention: } \mathbf{2 0 . 8 8} \pm \mathbf{7 . 7 7} \\
\text { Traditional therapy group } \\
\text { Pre-intervention: } \mathbf{2 9 . 4 5} \pm \mathbf{1 6 . 2 2} \\
\text { Post-intervention: } \mathbf{2 6 . 6 1} \pm \mathbf{1 2 . 9 2}\end{array}$ \\
\hline $\begin{array}{l}\text { Liao et al. } \\
2014 \\
\text { China } \\
{[120]}\end{array}$ & $\begin{array}{l}\text { Patients with } \\
\text { Parkinson's disease } \\
\text { (stages } 1 \text { to } 2 \text { on } \\
\text { Hoehn \& Yahr scale; } \\
\text { F/M, N=19/17) } \\
\text { Mean age: } 66 \pm 7 \text { yrs }\end{array}$ & $\begin{array}{l}\text { Intervention (RCT) } \\
3 \text { groups: Wii Fit vs. } \\
\text { traditional therapy } v s \text {. } \\
\text { passive control } \\
\text { Duration: } 6 \text { weeks ( } 45 \\
\text { min, } 2 \text { sessions/wk }+ \\
\text { follow-up after } 1 \\
\text { month } \\
\text { Location: } \\
\text { Rehabilitation center } \\
\text { (supposedly: } \\
\text { "administrated by the } \\
\text { same physical } \\
\text { therapist") }\end{array}$ & $\begin{array}{l}\text { Yoga }(10 \mathrm{~min}) \text { : Chair, } \\
\text { Sun Salutation, Tree; } \\
\text { strength }(15 \mathrm{~min}) \text {; } \\
\text { balance }(20 \mathrm{~min}) \text { : } \\
\text { Balance Bubble, } \\
\text { Soccer Heading, Ski } \\
\text { Slalom, Table Tilt }\end{array}$ & $\begin{array}{l}\text { Primary: mobility } \\
\text { (obstacle crossing } \\
\text { performance tests } \\
\text { measured with the } \\
\text { Liberty system), } \\
\text { dynamic balance } \\
\text { (LOS) } \\
\text { Secondary: balance } \\
\text { (SOT, FES), mobility } \\
\text { (TUG), quality of life } \\
\text { (PDQ-39). }\end{array}$ & $\begin{array}{l}\text { When compared with the passive } \\
\text { control group, Wii Fit induced } \\
\text { significant increases for the mobility, } \\
\text { balance and quality of life outcomes. } \\
\text { Interestingly, movement velocity } \\
\text { evaluated with LOS test showed } \\
\text { significantly greater improvement in the } \\
\text { Wii Fit group compared to traditional } \\
\text { therapy. These results should encourage } \\
\text { the implementation of Wii Fit activities } \\
\text { in patients with Parkinson's disease. } \\
\text { Adverse events: No } \\
\text { TUG (sec): } \\
\text { Wii Fit group } \\
\text { Pre- and post-intervention delta: -2.9 } \\
\pm \mathbf{2 . 2} \\
\text { Traditional therapy group } \\
\text { Pre- and post-intervention delta: } \mathbf{- 1 . 1} \\
\pm \mathbf{0 . 1}\end{array}$ \\
\hline
\end{tabular}




\begin{tabular}{|c|c|c|c|c|c|}
\hline & & & & & $\begin{array}{l}\text { Passive control group } \\
\text { Pre- and post-intervention delta: }+0.7 \\
\pm 1.7\end{array}$ \\
\hline $\begin{array}{l}\text { Morone et al. } \\
2014 \\
\text { Italy } \\
{[5]}\end{array}$ & $\begin{array}{l}\text { Subacute stroke } \\
\text { patients }(N=50) \\
\text { Mean age: } 60 \pm 10 \text { yrs }\end{array}$ & $\begin{array}{l}\text { Intervention (RCT) } \\
2 \text { groups: Wii Fit + } \\
\text { traditional therapy } v s . \\
\text { traditional balance } \\
\text { exercises + traditional } \\
\text { therapy } \\
\text { Duration: } 4 \text { wks }(20 \\
\text { min, } 3 \text { sessions/wk)+ } \\
\text { follow-up after } 1 \\
\text { month } \\
\text { Location: } \\
\text { Rehabilitation center }\end{array}$ & $\begin{array}{l}\text { Balance: Balance } \\
\text { Bubble, Ski Slalom; } \\
\text { aerobics: Hula Hoop }\end{array}$ & $\begin{array}{l}\text { Primary: balance } \\
\text { (BBS) } \\
\text { Secondary: mobility } \\
\text { (10-m walk test, } \\
\text { functional ambulatory } \\
\text { category), } \\
\text { independency (Barthel } \\
\text { index) }\end{array}$ & $\begin{array}{l}\text { Wii Fit was more effective than } \\
\text { traditional balance exercises to improve } \\
\text { balance and independency in subacute } \\
\text { stroke patients. No significant } \\
\text { differences were noted between groups } \\
\text { for mobility outcomes (increase in both } \\
\text { groups). Interestingly, benefits in } \\
\text { balance ability were maintained } 1 \\
\text { month after the intervention. } \\
\text { Adverse events: No }\end{array}$ \\
\hline $\begin{array}{l}\text { Omiyale et al. } \\
2015 \\
\text { USA } \\
{[92]}\end{array}$ & $\begin{array}{l}\text { Hemiparetic stroke } \\
\text { patients }(F / M, N=4 / 6 \\
\text { Age range: } 41-73 \mathrm{yrs} \\
\text { Mean age: } 67 \pm 8 \mathrm{yrs}\end{array}$ & $\begin{array}{l}\text { Intervention } \\
\text { I group } \\
\text { Duration: } 3 \text { wks (60 } \\
\text { min, } 3 \text { sessions/wk) } \\
\text { Location: not } \\
\text { specified, but } \\
\text { "supervised by a } \\
\text { physical therapist" }\end{array}$ & $\begin{array}{l}\text { Balance: Balance } \\
\text { Bubble, Penguin } \\
\text { Slide, Ski Slalom, } \\
\text { Table Tilt, Tightrope } \\
\text { Walk }\end{array}$ & $\begin{array}{l}\text { Neural plasticity } \\
\text { (interhemispheric } \\
\text { symmetry through } \\
\text { tibialis anterior } \\
\text { corticomotor } \\
\text { excitability using } \\
\text { transcranial magnetic } \\
\text { stimulation), balance, } \\
\text { motor response and } \\
\text { function (COP } \\
\text { distribution and } \\
\text { dynamic weight } \\
\text { shifting, Soccer } \\
\text { Heading's score, BBS, } \\
\text { TUG, and dual TUG, } \\
\text { gait speed, ABC) }\end{array}$ & $\begin{array}{l}\text { Interestingly, the Wii Fit intervention } \\
\text { significantly improved the } \\
\text { interhemispheric symmetry. Overall, but } \\
\text { not for all parameters, patients also } \\
\text { improved their balance abilities, motor } \\
\text { responsiveness, and balance related } \\
\text { functions. These results suggest that Wii } \\
\text { Fit rehabilitation may be able to } \\
\text { influence positively neural plasticity and } \\
\text { functional recovery in chronic stroke } \\
\text { patients. } \\
\text { Adverse events: No } \\
\text { ABC (no unit): } \\
\text { Wii Fit group } \\
\text { Pre-intervention: } \mathbf{6 5 . 9} \pm \mathbf{1 3 . 4 9} \\
\text { Post-intervention: } \mathbf{7 3 . 4} \pm \mathbf{1 3 . 3 2} \\
\text { BBS (no unit): } \\
\text { Wii Fit group } \\
\text { Pre-intervention: } \mathbf{5 1 . 6} \pm \mathbf{5 . 9 7} \\
\text { Post-intervention: } \mathbf{5 3 . 6} \pm \mathbf{2 . 9 5} \\
\text { TUG (sec): } \\
\text { Wii Fit group } \\
\text { Pre-intervention: } \mathbf{2 1 . 0} \pm \mathbf{1 2 . 1 8} \\
\text { Post-intervention: } \mathbf{1 9 . 4} \pm \mathbf{9 . 1 0}\end{array}$ \\
\hline
\end{tabular}




\begin{tabular}{|c|c|c|c|c|c|}
\hline $\begin{array}{l}\text { Yatar \& } \\
\text { Yildirim } \\
2015 \\
\text { Cyprus/Turkey } \\
{[19]}\end{array}$ & $\begin{array}{l}\text { Chronic stroke } \\
\text { patients }(F / M, \\
N=13 / 17) \\
\text { Mean age: } 60 \pm 14 \text { yrs }\end{array}$ & $\begin{array}{l}\text { Intervention (RCT) } \\
\text { 2 groups: } \\
\text { neurodevelopmental } \\
\text { training + Wii Fit vs. } \\
\text { neurodevelopmental } \\
\text { training + progressive } \\
\text { balance training } \\
\text { Duration: } 4 \text { wks (30 } \\
\text { min, } 3 \text { sessions/wk) } \\
\text { Location: } \\
\text { Rehabilitation center } \\
\text { (supposedly) }\end{array}$ & $\begin{array}{l}\text { Balance: } \text { Balance } \\
\text { Bubble, Ski Slalom, } \\
\text { Soccer Heading }\end{array}$ & $\begin{array}{l}\text { Primary: static } \\
\text { balance (Wii Balance } \\
\text { Board-assisted } \\
\text { postural sway tests), } \\
\text { dynamic balance } \\
\text { (BBS, DGI, functional } \\
\text { reach test, TUG) } \\
\text { Secondary: balance } \\
\text { confidence (ABC, } \\
\text { ADL) }\end{array}$ & $\begin{array}{l}\text { Primary and secondary outcomes } \\
\text { increased in both Wii Fit and } \\
\text { progressive balance training groups. } \\
\text { The increment was statistically higher in } \\
\text { the Wii Fit group for: DGI. Functional } \\
\text { reach test and ABC. Large differences } \\
\text { in baseline values between the two } \\
\text { groups limits the interpretation. } \\
\text { Adverse events: No } \\
\\
\text { BBS (no unit): } \\
\text { Wii Fit group } \\
\text { Pre-intervention: } \mathbf{4 5 . 6 0} \pm \mathbf{5 . 2 6} \\
\text { Post-intervention: } \mathbf{5 0 . 3 3} \pm \mathbf{4 . 0 9} \\
\text { Traditional therapy group } \\
\text { Pre-intervention: } \mathbf{3 9 . 6 0} \pm \mathbf{9 . 3 1} \\
\text { Post-intervention: } \mathbf{4 4 . 8 0} \pm 7.48 \\
\text { TUG (sec): } \\
\text { Wii Fit group } \\
\text { Pre-intervention: } \mathbf{1 7 . 9 6} \pm \mathbf{7 . 7 7} \\
\text { Post-intervention: } \mathbf{1 6 . 1 7} \pm \mathbf{8 . 2 3} \\
\text { Traditional therapy group } \\
\text { Pre-intervention: } \mathbf{2 6 . 3 6} \pm \mathbf{1 1 . 6 0} \\
\text { Post-intervention: } \mathbf{2 2 . 1 1} \pm \mathbf{1 1 . 8 8}\end{array}$ \\
\hline \multicolumn{6}{|c|}{ Orthopedic population } \\
\hline $\begin{array}{l}\text { Fung et al. } \\
2011 \\
\text { Canada } \\
{[11]}\end{array}$ & $\begin{array}{l}\text { Adult outpatients } \\
\text { following knee } \\
\text { replacement }(F / M, \\
N=33 / 17) \\
\text { Mean age: } 68 \pm 11 \mathrm{yrs}\end{array}$ & $\begin{array}{l}\text { Intervention }(\mathrm{RCT}) \\
2 \text { groups: traditional } \\
\text { therapy + Wii Fit vs. } \\
\text { traditional therapy }+ \\
\text { additional lower limb } \\
\text { exercise } \\
\text { Duration: until } \\
\text { discharge ( } \approx 6 \mathrm{wks}, 15 \\
\text { min/session) } \\
\text { Location: } \\
\text { Rehabilitation center }\end{array}$ & $\begin{array}{l}\text { Yoga: Deep } \\
\text { Breathing, Half } \\
\text { Moon; Strength: } \\
\text { Torso Twist; } \\
\text { Aerobics: Hula Hoop; } \\
\text { Balance: Balance } \\
\text { Bubble, Penguin } \\
\text { Slide, Ski Slalom, } \\
\text { Table tilt, Tightrope } \\
\text { Walk }\end{array}$ & $\begin{array}{l}\text { Function (range of } \\
\text { motion), 2-min walk } \\
\text { test, LEFS), pain } \\
\text { (NPRS), Balance } \\
\text { confidence (ABC) and } \\
\text { length of rehabilitation }\end{array}$ & $\begin{array}{l}\text { From baseline to discharge, the } \\
\text { improvements were similar between the } \\
\text { two groups for all the outcomes. The } \\
\text { Wii Fit intervention might induce higher } \\
\text { improvement for the LEFS. But the } \\
\text { study was not powerful enough to } \\
\text { obtain significance. } \\
\text { Adverse events: No }\end{array}$ \\
\hline $\begin{array}{l}\text { Baltaci et al. } \\
2012 \\
\text { Turkey } \\
{[72]}\end{array}$ & $\begin{array}{l}\text { Young adults with } \\
\text { anterior cruciate } \\
\text { ligament } \\
\text { reconstruction }(\mathrm{N}=30)\end{array}$ & $\begin{array}{l}\text { Intervention (RCT) } \\
2 \text { groups: Wii vs. } \\
\text { conventional } \\
\text { rehabilitation }\end{array}$ & $\begin{array}{l}\text { Not clear. Probably a } \\
\text { combination of Wii } \\
\text { Sports games } \\
\text { (Bowling, Boxing) }\end{array}$ & $\begin{array}{l}\text { Balance (SEBT), } \\
\text { function (functional } \\
\text { squat test including } \\
\text { coordination, }\end{array}$ & $\begin{array}{l}\text { No difference between Wii Fit and } \\
\text { conventional physical therapy. Wii Fit } \\
\text { may be able to address rehabilitation } \\
\text { goals for patients with anterior cruciate }\end{array}$ \\
\hline
\end{tabular}




\begin{tabular}{|c|c|c|c|c|c|}
\hline & Mean age: $29 \pm 5$ yrs & $\begin{array}{l}\text { Duration: } 12 \mathrm{wks}(60 \\
\text { min, } 3 \text { sessions/wk) } \\
\text { Location: } \\
\text { Rehabilitation center } \\
\text { (supposedly) }\end{array}$ & $\begin{array}{l}\text { and Wii Fit activities } \\
\text { ("skiing games", } \\
\text { "football", "balance } \\
\text { board") }\end{array}$ & $\begin{array}{l}\text { proprioception, time } \\
\text { response and strength } \\
\text { measurements) }\end{array}$ & $\begin{array}{l}\text { ligament reconstruction. } \\
\text { Adverse events: No }\end{array}$ \\
\hline $\begin{array}{l}\text { Sims et al. } \\
2013 \\
\text { USA } \\
{[74]}\end{array}$ & $\begin{array}{l}\text { Young active adults } \\
\text { with an history of } \\
\text { lower limb injury } \\
\text { within } 1 \mathrm{yr}(F / M \text {, } \\
N=16 / 12) \\
\text { Mean age: } 22 \pm 2 \text { yrs }\end{array}$ & $\begin{array}{l}\text { Intervention (RCT) } \\
3 \text { groups: Wii Fit, } \\
\text { traditional balance } \\
\text { training, passive } \\
\text { control } \\
\text { Duration: } 4 \text { wks } \\
\text { (15min, } 3 \\
\text { sessions/wk) } \\
\text { Location: } \\
\text { Rehabilitation center }\end{array}$ & $\begin{array}{l}\text { Yoga: Chair, Half } \\
\text { Moon, Tree; Balance: } \\
\text { Balance bubble, } \\
\text { Penguin Slide, Ski } \\
\text { Slalom, Soccer } \\
\text { Heading, Table Tilt; } \\
\text { Strength: Lunge, } \\
\text { Sideways Leg Lift, } \\
\text { Single Leg Extension; } \\
\text { Aerobics: Basic Step, } \\
\text { Hula Hoop, Super } \\
\text { Hula Hoop }\end{array}$ & $\begin{array}{l}\text { Primary: balance } \\
\text { (static: Time to } \\
\text { Boundary test, } \\
\text { dynamic: SEBT) } \\
\text { Secondary: function } \\
\text { (LEFS) }\end{array}$ & $\begin{array}{l}\text { Wii Fit improved static balance to a } \\
\text { larger extend than the traditional } \\
\text { balance training. Dynamic balance was } \\
\text { improved in all groups. } \\
\text { Adverse events: No }\end{array}$ \\
\hline $\begin{array}{l}\text { Punt et al. } \\
2015 \\
\text { Switzerland } \\
{[75]}\end{array}$ & $\begin{array}{l}\text { Adults ankle sprain } \\
\text { patients }(F / M, \\
N=39 / 51) \\
\text { Mean age: } 34 \pm 11 \text { yrs }\end{array}$ & $\begin{array}{l}\text { Intervention (RCT) } \\
3 \text { groups: Wii Fit vs. } \\
\text { traditional balance } \\
\text { training vs. passive } \\
\text { control } \\
\text { Duration: } 6 \text { wks ( } 30 \\
\text { min, } 2 \text { sessions/wk) } \\
\text { Location: } \text { Home }\end{array}$ & $\begin{array}{l}\text { Balance: Balance } \\
\text { Bubble, Penguin } \\
\text { Slide, Ski Slalom, } \\
\text { Table Tilt }\end{array}$ & $\begin{array}{l}\text { Function (FAAM), } \\
\text { pain (visual analogue } \\
\text { scale), time to return } \\
\text { to sport, satisfaction } \\
\text { (questionnaire) }\end{array}$ & $\begin{array}{l}\text { Foot and ankle ability score increased } \\
\text { and pain decreased in all groups. A Wii } \\
\text { Fit intervention was as effective as } \\
\text { traditional therapy or no therapy. In the } \\
\text { Wii Fit group, the average time to return } \\
\text { to sport was } 27 \pm 20 \text { days and } 82 \% \text { of } \\
\text { patients were satisfied. } \\
\text { Adverse events: No }\end{array}$ \\
\hline \multicolumn{6}{|c|}{ Multiple sclerosis } \\
\hline $\begin{array}{l}\text { Brichetto et al. } \\
2012 \\
\text { Italia } \\
{[81]}\end{array}$ & $\begin{array}{l}\text { Patients with multiple } \\
\text { sclerosis }(F / M, \\
N=22 / 14) \\
\text { Mean age: } 42 \pm 11 \mathrm{yrs}\end{array}$ & $\begin{array}{l}\text { Intervention (RCT) } \\
2 \text { groups: Wii Fit vs. } \\
\text { traditional } \\
\text { rehabilitation } \\
\text { Duration: } 4 \text { wks ( } 60 \\
\text { min, } 3 \text { sessions/wk) } \\
\text { Location: } \\
\text { Rehabilitation center }\end{array}$ & $\begin{array}{l}\text { Balance : Lotus } \\
\text { Focus, Ski Slalom, } \\
\text { Snowboard Slalom, } \\
\text { Soccer Heading, } \\
\text { Table Tilt, Tightrope } \\
\text { Walk }\end{array}$ & $\begin{array}{l}\text { Primary: balance } \\
\text { (BBS) } \\
\text { Secondary: fatigue } \\
\text { (MFIS), posture } \\
\text { (stabilometry) }\end{array}$ & $\begin{array}{l}\text { More important balance improvements } \\
\text { in the Wii Fit group. Fatigue was } \\
\text { reduced and posture improved. A Wii } \\
\text { Fit-based program might be more } \\
\text { efficient than the standard rehabilitation } \\
\text { procedure in multiple sclerosis patients. } \\
\text { Adverse events: No } \\
\text { BBS (no unit): } \\
\text { Wii Fit group } \\
\text { Pre-intervention: } \mathbf{4 9 . 6} \pm \mathbf{4 . 9} \\
\text { Post-intervention: } \mathbf{5 4 . 6} \pm \mathbf{2 . 2} \\
\text { Traditional therapy group } \\
\text { Pre-intervention: } \mathbf{4 8 . 7} \mathbf{3 . 3}\end{array}$ \\
\hline
\end{tabular}




\begin{tabular}{|c|c|c|c|c|c|}
\hline & & & & & Post-intervention: $49.7 \pm 3.9$ \\
\hline $\begin{array}{l}\text { Nilsagard et } \\
\text { al. } \\
2013 \\
\text { Sweden } \\
{[82]}\end{array}$ & $\begin{array}{l}\text { Patients with multiple } \\
\text { sclerosis }(F / M, \\
N=64 / 20) \\
\text { Mean age: } 50 \pm 11 \mathrm{yrs}\end{array}$ & $\begin{array}{l}\text { Intervention (RCT) } \\
2 \text { groups: Wii Fit vs. } \\
\text { passive control } \\
\text { Duration: } 6-7 \text { wks ( } 30 \\
\text { min, } 2 \text { sessions /wk) } \\
\text { Location: } \\
\text { Rehabilitation Center } \\
\text { ("physical therapist } \\
\text { supervised session") }\end{array}$ & $\begin{array}{l}\text { Aerobics: Skateboard } \\
\text { Arena } \\
\text { Balance: Balance } \\
\text { Bubble, Penguin } \\
\text { Slide, Ski Slalom, } \\
\text { Soccer Heading, } \\
\text { Snowboard Slalom, } \\
\text { Table Tilt, Tightrope } \\
\text { Walk; Training plus: } \\
\text { Balance Bubble plus, } \\
\text { Perfect 10, Table Tilt } \\
\text { Plus }\end{array}$ & $\begin{array}{l}\text { Primary: balance } \\
\text { (TUG) } \\
\text { Secondary: other } \\
\text { functional tests } \\
\text { (TUG }_{\text {cognitive, four }} \\
\text { square step test, } 25- \\
\text { foot walk test, DGI, } \\
\text { MSWS-12, ABC, } \\
\text { STST) }\end{array}$ & $\begin{array}{l}\text { Improvement in several balance-related } \\
\text { outcomes for the Wii Fit group. } \\
\text { However, same improvements were } \\
\text { observed in the controls (because of } \\
\text { spontaneous exercise). Wii Fit can be } \\
\text { recommended in adults with multiple } \\
\text { sclerosis } \\
\text { Adverse events: No } \\
\text { ABC (no unit): } \\
\text { Wii Fit group } \\
\text { Pre- and post-intervention delta: } \mathbf{5 . 0} \\
\pm \mathbf{1 4 . 4} \\
\text { TUG (sec): } \\
\text { Wii Fit group } \\
\text { Pre- and post-intervention delta: } \mathbf{- 0 . 8} \\
\pm \mathbf{2 . 4}\end{array}$ \\
\hline $\begin{array}{l}\text { Prosperini et } \\
\text { al. } \\
2013 \\
\text { Italy } \\
{[84]}\end{array}$ & $\begin{array}{l}\text { Patients with multiple } \\
\text { sclerosis }(F / M \text {, } \\
N=25 / 11) \\
\text { Mean age: } 36 \pm 9 \text { yrs }\end{array}$ & $\begin{array}{l}\text { Intervention (cross- } \\
\text { over RCT) } \\
2 \text { groups: Wii Fit vs. } \\
\text { passive control } \\
\text { Duration: } 12 \mathrm{wks}(30 \\
\text { min, } 5 \text { sessions/wk) } \\
\text { Location: Home }\end{array}$ & $\begin{array}{l}\text { Supposedly all } \\
\text { balance activities } \\
\text { included in Wii Fit } \\
\text { Plus (for the } 4 \text { first } \\
\text { weeks, patients were } \\
\text { allowed to play } \\
\text { Zazen, Table Tilt and } \\
\text { Ski Slalom only) }\end{array}$ & $\begin{array}{l}\text { Balance (COP } \\
\text { excursion, 4-step } \\
\text { square test), mobility } \\
\text { (25-foot walk test), } \\
\text { self-perceived } \\
\text { disability (MSIS-29) }\end{array}$ & $\begin{array}{l}\text { Wii Fit } \text { was effective in improving } \\
\text { balance, mobility and self-perceived } \\
\text { health status and quality of life. } \\
\text { Adverse events: knee pain }(\mathrm{N}=2) \text {, lower } \\
\text { back pain }(\mathrm{N}=3)\end{array}$ \\
\hline $\begin{array}{l}\text { Robinson et al. } \\
2015 \\
\text { UK } \\
{[86]}\end{array}$ & $\begin{array}{l}\text { Patients with multiple } \\
\text { sclerosis }(F / M, \\
N=38 / 18) \\
\text { Mean age: } 52 \pm 6 \text { yrs }\end{array}$ & $\begin{array}{l}\text { Intervention (RCT) } \\
3 \text { groups: Wii Fit vs. } \\
\text { traditional therapy vs. } \\
\text { passive control } \\
\text { Duration: } 4 \text { wks (40- } \\
60 \text { min, } 2 \\
\text { sessions/wk) } \\
\text { Location: } \\
\text { Rehabilitation center } \\
\end{array}$ & $\begin{array}{l}\text { Balance: Heading } \\
\text { Soccer, Ski Slalom, } \\
\text { Table Tilt, Tightrope } \\
\text { Walk; Strength: } \\
\text { Rowing Squats, Torso } \\
\text { Twist; aerobics: } \\
\text { Boxing, Hula Hoop, } \\
\text { Advanced Steps } \\
\text { (supposedly) }\end{array}$ & $\begin{array}{l}\text { Primary: balance } \\
\text { (postural sway), gait } \\
\text { (gait speed), } \\
\text { acceptability } \\
\text { (UTAUT, FSS) } \\
\text { Secondary: self- } \\
\text { perceived disability } \\
\text { (MSWS-12, } \\
\text { WHODAS) }\end{array}$ & $\begin{array}{l}\text { Balance but not gait was improved by } \\
\text { both the Wii Fit and traditional therapy } \\
\text { interventions. Wii Fit was acceptable } \\
\text { and induced positive changes in self- } \\
\text { perceived disability. } \\
\text { Adverse events: No }\end{array}$ \\
\hline \multicolumn{6}{|c|}{ Spinal cord injury patients } \\
\hline $\begin{array}{l}\text { Wall et al. } \\
2015 \\
\text { USA } \\
{[6]}\end{array}$ & $\begin{array}{l}\text { Individuals with } \\
\text { incomplete spinal } \\
\text { cord injury }(>1-\mathrm{yr} \\
\text { post-injury, } M, N=5)\end{array}$ & $\begin{array}{l}\text { Intervention } \\
1 \text { group } \\
\text { Duration: } 7 \mathrm{wk}(60 \\
\text { min, } 2 \text { sessions } / \mathrm{wk})+\end{array}$ & $\begin{array}{l}\text { Balance: Balance } \\
\text { Bubble, Basic Run (or } \\
\text { another running } \\
\text { activity), Penguin }\end{array}$ & $\begin{array}{l}\text { Walking ability (gait } \\
\text { speed), balance (TUG, } \\
\text { functional reach), } \\
\text { well-being (SF-36) }\end{array}$ & $\begin{array}{l}\text { Gait speed and functional reach tests } \\
\text { score both significantly increased after } \\
\text { the Wii Fit intervention and the effects } \\
\text { were maintained at a 4-week follow-up. }\end{array}$ \\
\hline
\end{tabular}




\begin{tabular}{|c|c|c|c|c|c|}
\hline & $\begin{array}{l}\text { Age range: } 50-64 \mathrm{yrs} \\
\text { Mean age: } 59 \pm 5 \mathrm{yrs}\end{array}$ & $\begin{array}{l}\text { follow up after } 4 \text { wks } \\
\text { Location: Home or } \\
\text { University (and } \\
\text { "supervised") }\end{array}$ & $\begin{array}{l}\text { Slide, Ski Slalom, Ski } \\
\text { Jump, Tightrope } \\
\text { Walk, Table Tilt; } \\
\text { training plus: Island } \\
\text { Bike, Obstacle } \\
\text { Course, Segway } \\
\text { Circuit, }\end{array}$ & & $\begin{array}{l}\text { However, the program failed to induce } \\
\text { statistical improvements in wellness } \\
\text { (SF-36) and TUG. } \\
\text { Adverse events: No }\end{array}$ \\
\hline \multicolumn{6}{|c|}{ Children with developmental delay } \\
\hline $\begin{array}{l}\text { Salem et al. } \\
2012 \\
\text { USA } \\
{[12]}\end{array}$ & $\begin{array}{l}\text { Children with } \\
\text { developmental delay } \\
(F / M, N=18 / 22) \\
\text { Age range: } 39 \text { to } 58 \\
\text { mths } \\
\text { Mean age: } 49 \pm 6 \text { mths }\end{array}$ & $\begin{array}{l}\text { Intervention (RCT) } \\
2 \text { groups: Wii vs. } \\
\text { passive control } \\
\text { Duration: } 10 \mathrm{wks} \\
\text { (30min, } 2 \\
\text { sessions/wk) } \\
\text { Location: } \\
\text { Rehabilitation center }\end{array}$ & $\begin{array}{l}\text { Strength: Lunges, } \\
\text { Single Leg Stance; } \\
\text { aerobics: Basic Run, } \\
\text { Basic Step, Hula } \\
\text { Hoop; balance: } \\
\text { Penguin Slide, Soccer } \\
\text { Heading, Tightrope } \\
\text { Walk; Wii Sports' } \\
\text { Baseball, Bowling } \\
\text { and Boxing games } \\
\text { were also used }\end{array}$ & $\begin{array}{l}\text { Primary: balance and } \\
\text { gait (gait speed, TUG, } \\
\text { single leg stance test }{ }^{\mathrm{a}} \text {, } \\
\text { STST, TUDS, 2-min } \\
\text { walk test) } \\
\text { Secondary: grip } \\
\text { strength } \\
\text { (dynamometer) }\end{array}$ & $\begin{array}{l}\text { Wii Fit induced significant } \\
\text { improvements for the single leg stance } \\
\text { test and grip strength only } \\
\text { Adverse events: No }\end{array}$ \\
\hline $\begin{array}{l}\text { Esposito et al. } \\
2013 \\
\text { Italia } \\
{[31]}\end{array}$ & $\begin{array}{l}\text { Children with } \\
\text { migraine without aura } \\
(F / M, N=32 / 39) \\
\text { Mean age: } 9 \pm 2 \text { yrs }\end{array}$ & $\begin{array}{l}\text { Intervention } \\
2 \text { groups: patients } v s . \\
\text { healthy controls, } F / M \text {, } \\
N=44 / 49 \text { ) } \\
\text { Duration: } 12 \text { wks ( } 30 \\
\text { min, } 3 \text { sessions/wk) } \\
\text { Location: Home }\end{array}$ & $\begin{array}{l}\text { A choice of } 18 \\
\text { balance oriented Wii } \\
\text { Fit Plus activities } \\
\text { (e.g. Balance Bubble, } \\
\text { Hula Hoop, Obstacle } \\
\text { Course, Penguin } \\
\text { Slide, Rhythm } \\
\text { Activities, Segway } \\
\text { Circuit, Snowboard } \\
\text { Slalom, Skateboard } \\
\text { Arena, Ski Jump, Ski } \\
\text { Slalom, Soccer } \\
\text { Heading, Table Tilt, } \\
\text { Tilt City...) }\end{array}$ & $\begin{array}{l}\text { Motor coordination } \\
\text { (MABC), fine } \\
\text { visuomotricity (Berry- } \\
\text { VMI) }\end{array}$ & $\begin{array}{l}\text { 3-month Wii Fit training in children } \\
\text { with migraine without aura improved all } \\
\text { parameters: motor coordination } \\
\text { (including balance skills), and fine } \\
\text { visuomotricity. Wii Fit could be used in } \\
\text { this population to counterbalance } \\
\text { associated developmental delays or } \\
\text { other deleterious effects. } \\
\text { Adverse events: No }\end{array}$ \\
\hline $\begin{array}{l}\text { Ferguson et al. } \\
2013 \\
\text { South Africa } \\
{[27]}\end{array}$ & $\begin{array}{l}\text { Children with } \\
\text { developmental } \\
\text { coordination disorders } \\
(F / M, N=22 / 24) \\
\text { Age range: } 6-10 \text { yrs } \\
\text { Mean age: } 8 \pm 1 \text { yrs }\end{array}$ & $\begin{array}{l}\text { Intervention } \\
2 \text { groups: Wii Fit vs. } \\
\text { established } \\
\text { neuromotor task } \\
\text { training } \\
\text { Duration: } 6 \text { wks ( } 30 \\
\text { min, } 3 \text { sessions/wk) }\end{array}$ & $\begin{array}{l}18 \text { of the Wii Fit } \\
\text { games mimicking the } \\
\text { act of cycling, soccer, } \\
\text { skateboarding and } \\
\text { skiing or played with } \\
\text { the hand controller }\end{array}$ & $\begin{array}{l}\text { Motor coordination } \\
\text { (MABC), physical } \\
\text { fitness (functional } \\
\text { strength, strength } \\
\text { measured with } \\
\text { dynamometer, muscle } \\
\text { power sprint test, 20- }\end{array}$ & $\begin{array}{l}\text { Motor performance improved in the two } \\
\text { groups, but more important changes } \\
\text { were noted in the traditional training } \\
\text { group. The latter was also true for } \\
\text { functional strength and } \\
\text { cardiorespiratory fitness measurements. } \\
\text { Adherence was near } 100 \% \text { in both }\end{array}$ \\
\hline
\end{tabular}




\begin{tabular}{|c|c|c|c|c|c|}
\hline & & Location: School & & $\begin{array}{l}\mathrm{m} \text { shuttle run test, } \\
\text { adherence }\end{array}$ & $\begin{array}{l}\text { groups. The choice of one or the other } \\
\text { intervention may depend on resources } \\
\text { and time constraints. } \\
\text { Adverse events: No }\end{array}$ \\
\hline $\begin{array}{l}\text { Hammond et } \\
\text { al. } \\
2013 \\
\text { UK } \\
{[28]}\end{array}$ & $\begin{array}{l}\text { Children with } \\
\text { developmental } \\
\text { coordination disorders } \\
(F / M, N=4 / 14) \\
\text { Age range: } 7-10 \mathrm{yrs} \\
\text { Mean age: } 8 \pm 1 \text { yrs }\end{array}$ & $\begin{array}{l}\text { Intervention (cross- } \\
\text { over RCT) } \\
2 \text { groups: Wii Fit- } \\
\text { regular motor training } \\
\text { vs. regular motor } \\
\text { training-Wii Fit } \\
\text { Duration: } 4 \text { wks (3 } \\
\text { sessions/wk) } \\
\text { Location: School }\end{array}$ & $\begin{array}{l}\text { A selection of } 9 \mathrm{Wii} \\
\text { Fit games focusing on } \\
\text { coordination and } \\
\text { balance }\end{array}$ & $\begin{array}{l}\text { Childs: motor } \\
\text { proficiency (BOT), } \\
\text { self-perceived ability } \\
\text { and satisfaction with } \\
\text { motor task } \\
\text { (coordination skills } \\
\text { questionnaire) } \\
\text { Parents: emotional } \\
\text { and behavioral } \\
\text { development } \\
\text { (strengths and } \\
\text { difficulties } \\
\text { questionnaire) } \\
\end{array}$ & $\begin{array}{l}\text { Wii Fit induced significant gains in } \\
\text { motor proficiency and other outcomes } \\
\text { for many, but not all the children. } \\
\text { Including Wii Fit therapy for children } \\
\text { with developmental disorders could be } \\
\text { considered. } \\
\text { Adverse events: No }\end{array}$ \\
\hline $\begin{array}{l}\text { Mombarg et } \\
\text { al. } \\
2013 \\
\text { The } \\
\text { Netherland } \\
\text { [29] }\end{array}$ & $\begin{array}{l}\text { Children with balance } \\
\text { alterations }(F / M \text {, } \\
N=6 / 23) \\
\text { Age range: } 7-12 \mathrm{yrs} \\
\text { Mean age: } 10 \pm 1 \mathrm{yrs}\end{array}$ & $\begin{array}{l}\text { Intervention (RCT) } \\
2 \text { groups: Wii Fit vs. } \\
\text { passive control } \\
\text { Duration: } 6 \text { wks ( } 30 \\
\text { min, } 3 \text { sessions/wk) } \\
\text { Location: } \text { School }\end{array}$ & $\begin{array}{l}18 \text { activities identified } \\
\text { as balance games } \\
\text { (mainly from the } \\
\text { balance and training } \\
\text { plus categories: Ski } \\
\text { Jump, Ski Slalom, } \\
\text { Snowboard Slalom, } \\
\text { Table Tilt, Obstacle } \\
\text { Course, Segway } \\
\text { Circuit, Skateboard } \\
\text { Arena, Tilt City, } \\
\text { Rhythm activities...) }\end{array}$ & $\begin{array}{l}\text { Balance (MABC \& } \\
\text { BOT) }\end{array}$ & $\begin{array}{l}\text { Significant improvements in the Wii Fit } \\
\text { group. Effective intervention for } \\
\text { children with poor motor development. } \\
\text { Adverse events: No }\end{array}$ \\
\hline $\begin{array}{l}\text { Jelsma et al. } \\
2014 \\
\text { The } \\
\text { Netherlands } \\
{[30]}\end{array}$ & $\begin{array}{l}\text { Children with } \\
\text { probable } \\
\text { developmental } \\
\text { coordination disorders } \\
\text { and balance problems } \\
(F / M=10 / 18) \\
\text { Age range: } 71-136 \\
\text { mths } \\
\text { Mean age: } 100 \pm 15 \\
\text { mths }\end{array}$ & $\begin{array}{l}\text { Intervention } \\
2 \text { groups: Wii Fit vs. } \\
\text { waiting period-Wii Fit } \\
\text { Duration: } 6 \text { weeks ( } 30 \\
\text { min, } 3 \text { sessions/wk) } \\
\text { Location: Laboratory } \\
\text { (supposedly) }\end{array}$ & $\begin{array}{l}18 \text { "balancing } \\
\text { activities" from the } \\
\text { Wii Fit Plus software } \\
\text { (not including Ski } \\
\text { Slalom, which was } \\
\text { used for test) }\end{array}$ & $\begin{array}{l}\text { Motor coordination } \\
\text { (MABC), balance } \\
\text { (BOT, Ski Slalom), } \\
\text { enjoyment (home- } \\
\text { made scale) }\end{array}$ & $\begin{array}{l}\text { A Wii Fit intervention significantly } \\
\text { increased motor and balance skills in } \\
\text { children with coordination disorders and } \\
\text { balance problems. After } 6 \text { weeks of } \\
\text { intervention, } 20 \text { children (out of } 28 \text { ) still } \\
\text { rated Wii Fit as "super fun" and } 4 \text { as } \\
\text { "fun". } \\
\text { Adverse events: No }\end{array}$ \\
\hline
\end{tabular}




\begin{tabular}{|c|c|c|c|c|c|}
\hline $\begin{array}{l}\text { Ramstrand \& } \\
\text { Lygnegard } \\
2012 \\
\text { Sweden } \\
{[35]}\end{array}$ & $\begin{array}{l}\text { Children with } \\
\text { hemiplegic or diplegic } \\
\text { cerebral palsy }(F / M \text {, } \\
N=10 / 8) \\
\text { Age range: } 8-17 \mathrm{yrs} \\
\text { Mean age: } 13 \pm 3 \mathrm{yrs}\end{array}$ & $\begin{array}{l}\text { Intervention (cross- } \\
\text { over RCT) } \\
2 \text { groups: Wii Fit-no } \\
\text { intervention } v \text { s. no } \\
\text { intervention-Wii Fit } \\
\text { Duration: } 5 \text { wks } \\
\text { (>30min, } 5 \\
\text { sessions/wk) } \\
\text { Location: Home }\end{array}$ & $\begin{array}{l}\text { Balance: Balance } \\
\text { Bubble, Ski Jump, Ski } \\
\text { Slalom, Soccer } \\
\text { Heading, Table Tilt, } \\
\text { Tightrope Walk, }\end{array}$ & $\begin{array}{l}\text { Primary: balance } \\
\text { (modified SOT, } \\
\text { reactive balance test, } \\
\text { weight shift test) } \\
\text { Secondary: adherence } \\
\text { (playing time) }\end{array}$ & $\begin{array}{l}\text { No improvements after the Wii Fit } \\
\text { intervention period. A } 30 \text {-min home- } \\
\text { based Wii Fit intervention was not } \\
\text { effective to improve balance in children } \\
\text { with cerebral palsy. } 4 \text { children did not } \\
\text { complete the required } 30 \mathrm{~min} / \text { day } \\
\text { sessions. } \\
\text { Adverse events: No }\end{array}$ \\
\hline $\begin{array}{l}\text { Jelsma et al. } \\
2013 \\
\text { South Africa } \\
{[36]}\end{array}$ & $\begin{array}{l}\text { Children with spastic } \\
\text { hemiplegic cerebral } \\
\text { palsy }(F / M, N=6 / 8) \\
\text { Age range: } 7-14 \text { yrs } \\
\text { Mean age: } 11 \pm 2 \text { yrs }\end{array}$ & $\begin{array}{l}\text { Intervention (A-B) } \\
1 \text { group }(c f . \text { details in } \\
\text { the paper) } \\
\text { Duration: } 3 \text { wks } \\
\text { (25min, } 4 \\
\text { sessions/wk) + follow- } \\
\text { up after } 2 \text { months } \\
\text { Location: } \\
\text { Rehabilitation center }\end{array}$ & $\begin{array}{l}\text { Aerobics: Hula Hoop; } \\
\text { balance: Balance } \\
\text { Bubble, Penguin } \\
\text { Slide, Snowboard } \\
\text { Slalom, Slalom Ski, } \\
\text { Soccer Heading }\end{array}$ & $\begin{array}{l}\text { Balance (BOT), } \\
\text { functional mobility } \\
\text { (BOT and TUDS) }\end{array}$ & $\begin{array}{l}\text { Balance score improved significantly } \\
\text { (sustained at } 2 \text { months follow-up), but } \\
\text { not the functional scores (BOT and } \\
\text { TUDS). } 10 \text { children only preferred to } \\
\text { play Wii Fit instead of conventional } \\
\text { physiotherapy. Wii Fit may not be used } \\
\text { in place of conventional therapy. } \\
\text { Adverse events: No }\end{array}$ \\
\hline $\begin{array}{l}\text { Tarakci et al. } \\
2013 \\
\text { Turkey } \\
{[37]}\end{array}$ & $\begin{array}{l}\text { Children with } \\
\text { ambulatory cerebral } \\
\text { palsy }(F / M, N=3 / 11) \\
\text { Mean age: } 12 \pm 3 \text { yrs }\end{array}$ & $\begin{array}{l}\text { Intervention } \\
1 \text { group } \\
\text { Duration: } 12 \mathrm{wks} \\
\text { (40min, } 2 \\
\text { sessions/wk) } \\
\text { Location: } \\
\text { Rehabilitation center }\end{array}$ & $\begin{array}{l}\text { Balance: Ski Slalom, } \\
\text { Soccer Heading, } \\
\text { Table Tilt, Tightrope } \\
\text { Walk }\end{array}$ & $\begin{array}{l}\text { Balance (single leg } \\
\text { stance test }^{\mathrm{a}} \text {, functional } \\
\text { reach test, TUG, } 6- \\
\text { min walk test) }\end{array}$ & $\begin{array}{l}\text { Balance improved significantly (all } \\
\text { outcomes) } \\
\text { Adverse events: No } \\
\text { TUG (sec): } \\
\text { Wii Fit group } \\
\text { Pre-intervention: } \mathbf{1 8 . 2 6} \pm \mathbf{8 . 9 5} \\
\text { Post-intervention: } \mathbf{1 4 , 5 7} \pm \mathbf{5 . 3 9}\end{array}$ \\
\hline \multicolumn{6}{|c|}{ Other populations with balance impairments } \\
\hline $\begin{array}{l}\text { Meldrum et al. } \\
2015 \\
\text { Ireland } \\
{[21]}\end{array}$ & $\begin{array}{l}\text { Patients with } \\
\text { unilateral peripheral } \\
\text { vestibular loss }(F / M \text {, } \\
N=27 / 44) \\
\text { Mean age: } 54 \pm 15 \mathrm{yrs}\end{array}$ & $\begin{array}{l}\text { Intervention (RCT) } \\
2 \text { groups: Wii Fit vs. } \\
\text { traditional balance } \\
\text { rehabilitation } \\
\text { Duration: } 6 \text { wks }(15 \\
\text { min, } 5 \text { sessions/wk })+ \\
\text { follow-up after } 6 \\
\text { months } \\
\text { Location: Home }\end{array}$ & $\begin{array}{l}\text { Yoga: Deep } \\
\text { Breathing, Palm Tree, } \\
\text { Standing Knee, Tree } \\
\text { Pose; strength: } \\
\text { Sideways Leg Lift, } \\
\text { Single Leg Extension; } \\
\text { balance: Balance } \\
\text { Bubble, Ski Slalom, } \\
\text { Heading Soccer, } \\
\text { Penguin Slide, Table } \\
\text { Tilt; aerobics: } \\
\text { Advanced Step, Basic } \\
\text { Step, Free Step; } \\
\end{array}$ & $\begin{array}{l}\text { Primary: Gait (self- } \\
\text { preferred gait speed) } \\
\text { Secondary: gait } \\
\text { parameters (various } \\
\text { tests performed eyes } \\
\text { open or close, } \\
\text { including DGI), } \\
\text { balance (ABC, SOT), } \\
\text { dynamic visual acuity } \\
\text { (computerized } \\
\text { dynamic visual acuity } \\
\text { system), self- } \\
\text { perceived benefit }\end{array}$ & $\begin{array}{l}\text { Both the Wii Fit and traditional balance } \\
\text { training induced improvement in gait } \\
\text { speed. No difference was noted between } \\
\text { the two groups for gait parameters and } \\
\text { other outcomes at the end of the } \\
\text { intervention. Adherence was high for } \\
\text { the two interventions but Wii Fit } \text { was } \\
\text { described as more enjoyable. } \\
\text { Adverse events: low back pain }(\mathrm{N}=1) \\
\text { ABC (no unit): } \\
\text { Wii Fit group } \\
\text { Pre-intervention: } \mathbf{6 4 . 8 2} \pm \mathbf{1 8 . 7 4}\end{array}$ \\
\hline
\end{tabular}




\begin{tabular}{|l|l|l|l|l|}
\hline & & $\begin{array}{l}\text { training plus: } \\
\text { Skateboard, Snowball } \\
\text { Fight, Table Tilt Plus }\end{array}$ & $\begin{array}{l}\text { (vestibular } \\
\text { rehabilitation benefit } \\
\text { questionnaire), mental } \\
\text { health (Hospital } \\
\text { Anxiety and } \\
\text { depression Scale), } \\
\text { adherence (diary) }\end{array}$ & Post-intervention: 74.36 $\pm \mathbf{2 1 . 2 5}$ \\
& & & \\
\hline
\end{tabular}

a Many different single leg stance tests were used in the Wii Fit literature for balance assessment purposes. In this table "single leg stance test" describe any test requiring subjects to stand on one leg. ${ }^{b}$ Many different sit-to-stand tests (STST) were used in the Wii Fit literature for balance, strength or functional assessment purpose. In this table, "STST" describes any test that requires the subject to sit and stand repeatedly. ${ }^{\mathrm{c}}$ Esculier et al. (2012 \& 2014) report results obtained with the same group of subjects during the same trial [113, $118]$.

ABC: activities-specific balance confidence scale; BBS: Berg balance scale; BMI: body mass index; BOT: Bruininks-Oseretsky test; ADL: activities of daily living scale; CHAMPS: community healthy activities model program for seniors; CBM: community balance and mobility scale; COPD: chronic obstructive pulmonary disease; Beery-VMI: Beery visual-motor integration test; CRQ-SR: chronic respiratory questionnaire; CTSIB: clinical test of sensory interaction and balance; DGI: dynamic gait index; EE: energy expenditure; ESWT: endurance shuttle walk test; FAAB: foot and ankle ability measure; FAB: Fullerton advanced balance scale; FES: Falls Efficacy Scale; FIM: functional independence measure; FSS: flow state scale; GDS: geriatric depression scale; HR: hear rate (beats/min); IMI: Intrinsic Motivation Inventory; LASAPAQ: LASA Physical Activity Questionnaire; LEFS: lower extremity functional scale; LLFDI: late life function and disability index; LOS: limits of stability; MABC: movement assessment battery for children; MCTSIB: modified clinical test for sensory interaction in balance; MDRT: multidirectional reach test; METs: metabolic equivalent; MFIS: modified fatigue impact scale; MSIS-29: 29-item multiple sclerosis impact scale; MSWS-12: 12-items multiple sclerosis walking scale; MVC: maximal voluntary contraction of leg extensors; MVPA: moderate-to-vigorous physical activity; NPRS: numeric pain rating scale; OEE: Outcome expectations for exercise scale; PA: physical activity; PACES: physical activity and exercise questionnaire; PAID: problem areas in diabetes scale; PDQ-39: 39-question Parkinson's disease questionnaire; RFD: rate of force development; RPP: rate pressure product; SE: Schwab \& England daily living activities scales; SEE: self-efficacy exercise scale; SEES: subjective exercise experience scale; SF-8: short form-8 health survey; SF-36: short form-36 health survey; SFT: senior fitness test; SOT: sensory organization test; STREAM: stroke rehabilitation assessment of movement; STST: sit-to-stand-test; TD2M: type 2 diabetes mellitus; TUDS: time up and down stairs; TUG: time up and go; UPDRS: unified rating scale for Parkinson's disease; UTAUT: unified theory of acceptance and use of technology questionnaire; VPA: vigorous physical activity; $\mathrm{VO}_{2}$ : oxygen consumption; $\mathrm{VO}_{2}$ max: maximal oxygen consumption; WHO-5: five-item WHO well-being index; WHODAS: world health organization disability assessment schedule 


\section{PeerJ}

239 For the same test, unit may vary from one paper to another. 
Goal 3: Outcomes of Meta-analyses

241 For the pre- and post-intervention meta-analyses, 7 groups out of 6 studies were included for

242 ABC, 13 groups out of 12 studies for BBS, and 12 groups out of 12 studies for TUG. For the Wii

243 Fit vs. traditional therapy meta-analyses, 14 groups out of 7 studies for BBS, and 12 groups out

244 of 6 studies for TUG. Studies included in the different meta-analyses involved 595 participants

245 from both sexes (females: 332, males: 242, not specified: 21$)$, with a wide age range (12 $\pm 3-86$

$246 \pm 5$ years $[37,100])$ and various medical conditions. Whilst these papers all included a measure

247 of $\mathrm{ABC}, \mathrm{BBS}$ or TUG, the interventions content and duration vary from study to study. The

248 assessment of individual studies revealed a low risk of bias (Figure 2). Detailed results for ABC,

249 BBS and TUG are presented in Figure 3, 4 and 5, and data included in the meta-analyses appears

250 in Table 3 and 4. Wii Fit interventions did not induce any change in ABC (2.02, 95\% CI: $-4.01-$

251 8.04). For BBS, significant improvements were noted in both healthy subjects and patients (2.00,

252 95\% CI: $0.41-3.60$ and 2.99, 95\% CI: $0.08-5.90$, respectively; 2.23, 95\% CI: $0.84-3.63$,

253 overall). In addition, there was no significant difference in changes induced by traditional

254 training and those induced by Wii Fit, suggesting that Wii Fit was as valid as traditional training.

255 Regarding TUG, no significant reduction was noted after the Wii Fit intervention in either

256 healthy subjects or patients $(-0.34 \mathrm{sec}, 95 \% \mathrm{CI}:-1.38-0.70$ and $-2.24 \mathrm{sec}, 95 \% \mathrm{CI}:-5.17-0.69$,

257 respectively; $-0.55 \mathrm{sec}, 95 \% \mathrm{CI}:-1.53-0.43$, overall). However, compared to traditional training

258 programs, the Wii Fit did induced a more significant reduction in TUG, especially in patients (-

259 1.76, 95\% CI: $-2.13--1.39$, in patients; $-1.31,95 \%$ CI:-1.62 - -1.01, overall). The sets of studies

260 included in both BBS and TUG pre- and post-intervention meta-analyses were statistically

261 homogenous $\left(\mathrm{I}^{2}=0.0 \%, \mathrm{p}=0.961\right.$ and $\mathrm{I}^{2}=0.0 \%, \mathrm{p}=0.969$, respectively for the overall analysis $)$.

262 Various levels of heterogeneity were observed in the Wii Fit vs. traditional therapy meta-

263 analyses $\left(\mathrm{I}^{2}=60.0 \%, \mathrm{p}=0.040\right.$ for BBS in patients and $\mathrm{I}^{2}=74.3 \%, \mathrm{p}=0.002$ for TUG overall 
264 (Figure 4 and 5), indicating some inconsistencies in the literature. This was expected, however,

265 since different populations were included in the analyses.

266 Meta-regression analyses revealed no significant results (not shown), suggesting no relationships

267 between improvements in balance outcomes and intervention duration or volume.

268

269 


\section{Pre-/post- intervention changes for $A B C$}

Study ID

Effect $(95 \% \mathrm{Cl}) \quad$ Weight (\%)

Healthy subjects

Orsega-Smith, 2012(a)

Orsega-Smith, 2012(b)

Subtotal $\left(l^{2}=0.0 \%, p=0.854\right)$

Patients

Mhatre, 2013 (Parkinson's disease)

Nilsagard, 2013 (multiple sclerosis)

Hoffman, 2014 (post-surgery cancer patients)

Meldrum, 2015 (vestibular disorders)

Omiyale, 2015 (hemiparetic stroke patients)

Subtotal $\left(I^{2}=0.0 \%, p=0.835\right)$

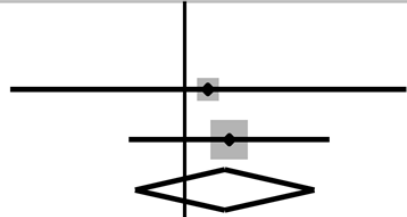

$3.20(-24.00,30.40) \quad 4.90$

$6.07(-7.73,19.87) \quad 19.05$

$5.48(-6.82,17.79) \quad 23.95$

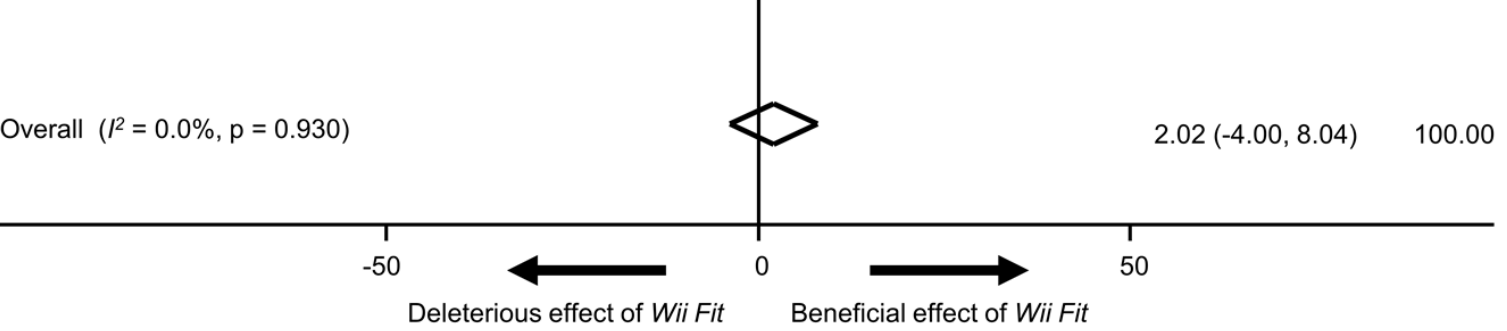

Figure 3. Pre- and post-intervention meta-analytic effect for the activities-specific balance describe the pooled values respectively for the change in healthy subjects, patients and the overall population. The vertical black line refers to no change. For each analysis (overall population) or sub-analysis (healthy subjects or patients), a significant effect is observed if the

276 diamond does not touch the black line. The horizontal black line shows the $95 \%$ confidence

277 interval and the grey square shows the study weight in percentage. Orsega et al. 2012(a): 4-week

278 Wii Fit intervention group; Orsega et al. 2012(b): 8-week Wii Fit intervention group [98]. $I^{2}$ :

279 index of heterogeneity. 


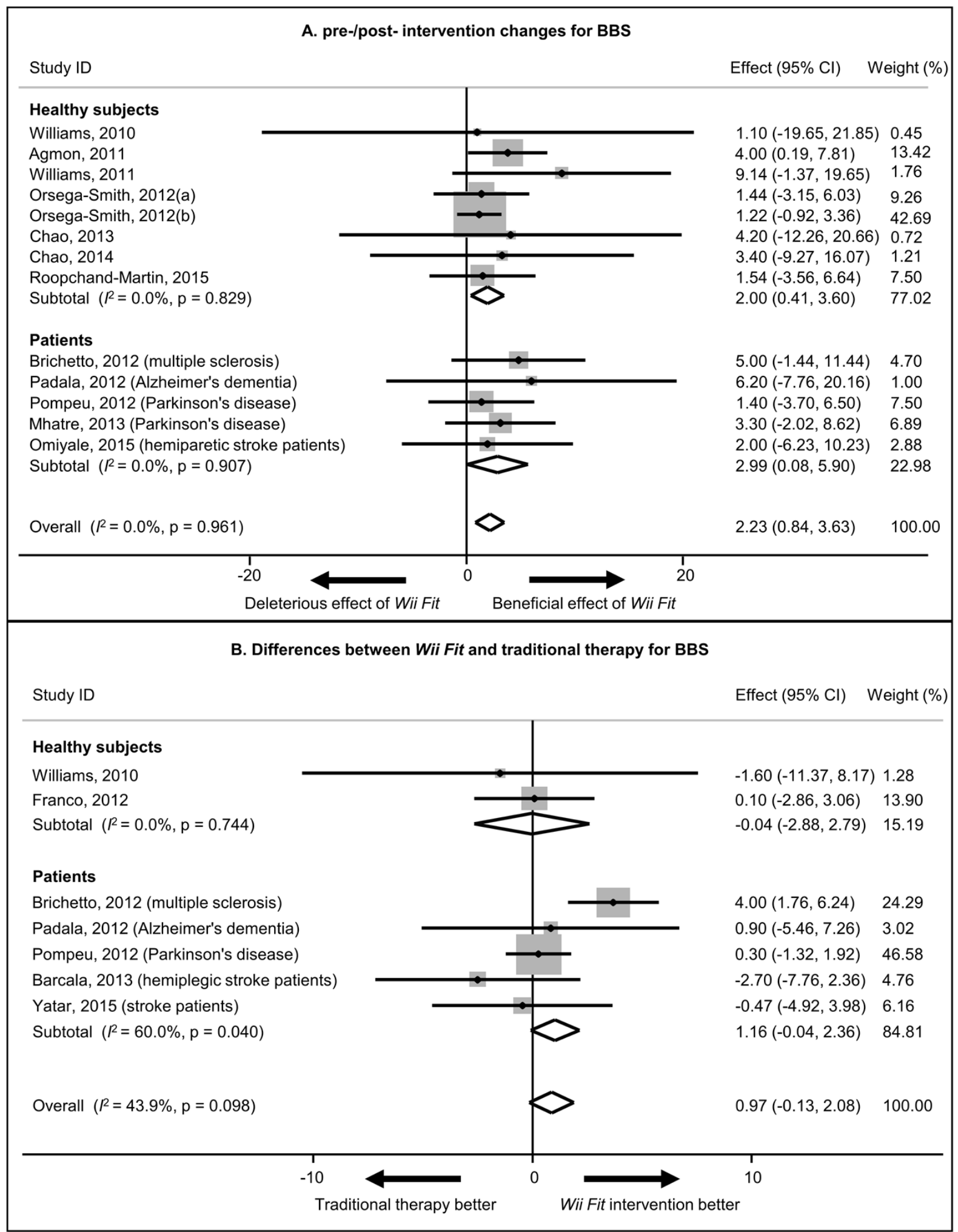

281 Figure 4. Pre- and post-intervention meta-analytic effect (A) and Wii-Fit vs. traditional

282 therapy meta-analytic effect (B) for the Berg balance score (BBS). A. The black point shows 
283 the average change for each study. The diamonds describe the pooled values respectively for the

284 change in healthy subjects, patients and the overall population. The vertical black line refers to

285 no change. For each analysis (overall population) or sub-analysis (healthy subjects or patients), a

286 significant effect is observed if the diamond does not touch the black line. B. The black point

287 shows the difference of effect between Wii Fit and traditional therapy for each study. The

288 diamonds describe the pooled values respectively for the difference of effect in healthy subjects,

289 patients and the overall population. The vertical black line refers to no difference between Wii

290 Fit-induced change and traditional therapy-induced change. For each analysis (overall

291 population) or sub-analysis (healthy subjects or patients), a significant difference is observed if

292 the diamond does not touch the black line. A and B. The horizontal black line shows the $95 \%$

293 confidence interval and the grey square shows the study weight in percentage. Orsega et al.

294 2012(a): 4-week Wii Fit intervention group; Orsega et al. 2012(b): 8-week Wii Fit intervention

295 group [98]. $I^{2}$ : index of heterogeneity. 


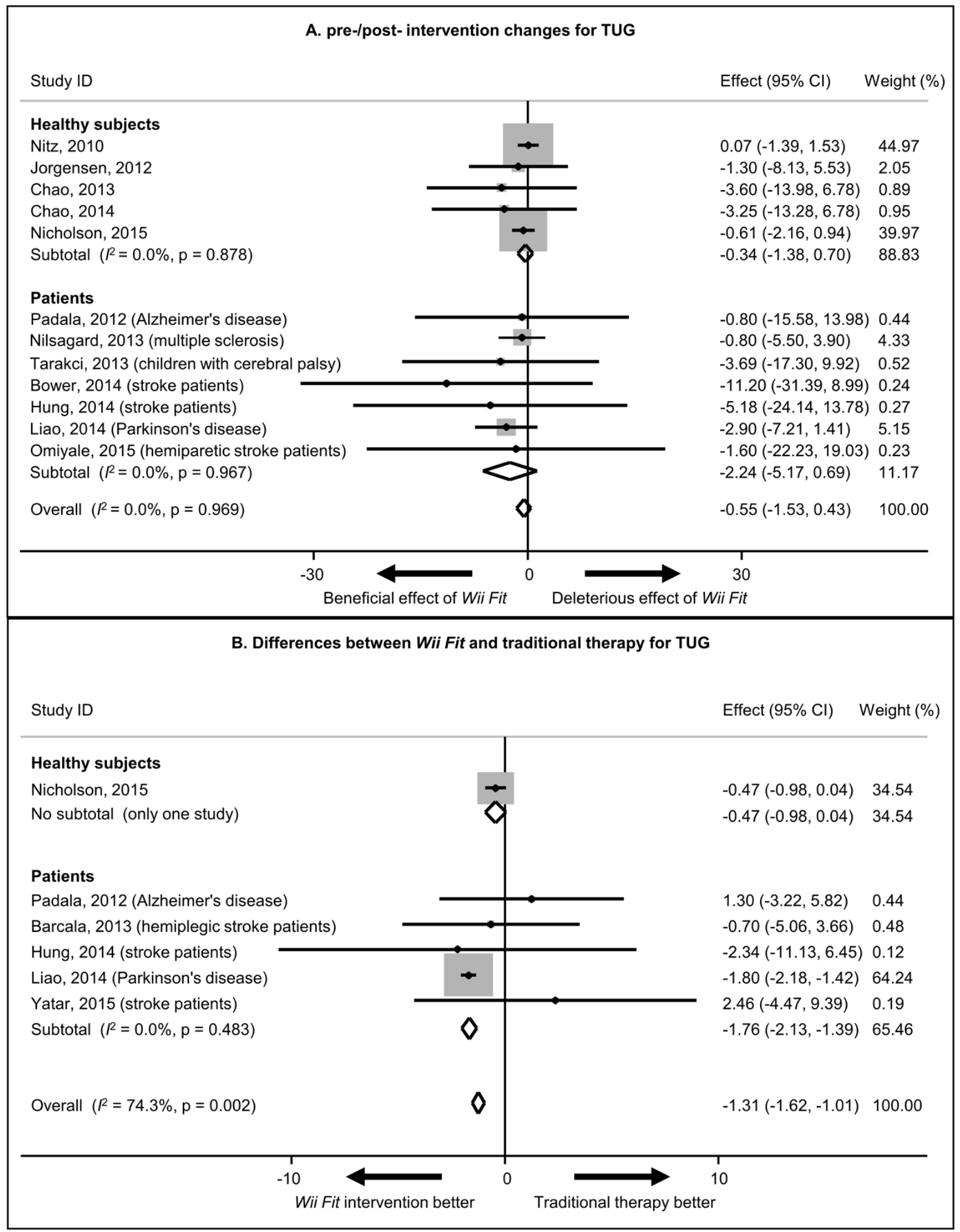

297 Figure 5. Pre- and post-intervention meta-analytic effect (A) and Wii-Fit vs. traditional

298 therapy meta-analytic effect (B) for the time-up-and-go test (TUG). A. The black point 
299 shows the average change for each study. The diamonds describe the pooled values respectively

300 for the change in healthy subjects, patients and the overall population. The vertical black line 301 refers to no change. For each analysis (overall population) or sub-analysis (healthy subjects or

302 patients), a significant effect is observed if the diamond does not touch the black line. B. The

303 black point shows the difference of effect between Wii Fit and traditional therapy for each study.

304 The diamonds describe the pooled values respectively for the difference of effect in healthy

305 subjects, patients and the overall population, the vertical black line refers to no difference

306 between Wii Fit-induced change and traditional therapy-induced change. For each analysis

307 (overall population) or sub-analysis (healthy subjects or patients), a significant difference is

308 observed if the diamond does not touch the black line. A and B. The horizontal black line shows

309 the $95 \%$ confidence interval and the grey square shows the study weight in percentage. $I^{2}$ : index

310 of heterogeneity. Unlike ABC and BBS, which are scores, the TUG test results are expressed in

311 time. A negative difference therefore indicates a higher performance. 


\section{Discussion}

313 The three main goals set for this review were as follows:

314 - Goal 1: Identify the health-related domains in which the Wii Fit series has been tested or used.

315 A scientific database search was undertaken with reasoned exclusion criteria. We identified

316 that the Wii Fit has been used for numerous health purposes and in various populations

317 (Table 2). Balance training was identified as being the most recurrent topic in the literature

318 and appears to be the field of predilection for the usage of the Wii Fit software. Another

319 notable focus was the prevention of metabolic disorders as well as the improvement of health

320 status in people with chronic disease.

321

- Goal 2: Understand the effect of Wii Fit in the identified populations (cf. Goal 1). A

322 qualitative systematic review of studies including Wii Fit interventions was performed, with

323 particular attention given to health and physical activity outcomes. Wii Fit was employed to

324 prevent falls, to induce functional improvements in seniors or in subjects presenting

325 neurodegenerative diseases, to treat orthopedic populations, etc. (Table 4). Overall, the

326 effects of using Wii Fit were mainly positive, with the software being recurrently described

327 as being able to induce similar benefits to traditional therapies. In addition, Wii-Fit

328 interventions were linked to an improvement of health status in several different patients

329 types (diabetic subjects, cancer patients...), however its preventive effect remains to be

330 demonstrated.

- Goal 3: To conduct meta-analyses when possible to quantify the effect Wii Fit had on selected health-related domains. In regards to balance training, the results of meta-analyses revealed that Wii Fit interventions had a positive impact on BBS and TUG. Interestingly, Wii

334 Fit interventions also appear very safe, with very low levels of injuries being reported. 
337 From light physical activity to moderate-to-vigorous physical activity, AVG elicit a wide range of intensities [23, 26, 41, 44, 45, 46, 48, 55, 56, 62, 64-66, 123]. However, it is difficult to state whether playing Wii Fit on a regular basis would allow one to meet the American College of Sports Medicine's recommendations for physical activity or could induce beneficial effects on health. Intervention studies reviewed in this article indicate that playing Wii Fit is not a strategy to consider in young adults (and children) for the prevention of cardio-metabolic disease, because it does not induce any significant increase in physical activity or any improvement in physical fitness $[24,60]$. However, one study showed a significant and rapid weight loss during a Wii Fit intervention in postpartum women [64]. Wii Fit may also be a promising tool to aid seniors in maintaining a healthy lifestyle. Intervention studies have reported an increase in physical activity [8], physical fitness [18], and functional skills [110]. Playing Wii Fit also clearly appeared to be beneficial for various types of patients: Some studies have reported improvements to health status in chronic obstructive pulmonary disease, hemodialysis patients, diabetic subjects, and cancer patients [77-79, 87, 88]. While the preventive effects of Wii Fit remain to be demonstrated, the software may be of value in other clinical settings.

Many of the intervention studies (55/68) were related to balance training or to the improvement of related functions, with a large majority of them (50/55, Table 4) describing a beneficial effect.

356 The meta-analytic results supported these promising observations. Significant improvements 
357 were observed for BBS in both healthy subjects and patients, while a trend was noted for TUG

358 improvements in patients. Interestingly, the meta-analyses also revealed no difference in

359 improvements induced by traditional therapies and Wii Fit interventions for BBS, while TUG

360 showed greater improvements following the Wii Fit intervention compared to after traditional

361 therapy. Taken together, these outcomes suggest a possible therapeutic application for the

362 software, with Wii Fit potentially being as valid as traditional training in some situations.

363 However, a careful look at the qualitative analysis outcome (Table 4) mitigates the overall

364 positive impression for some populations. For instance, Wii Fit intervention outcomes in children

365 with cerebral palsy appeared somewhat contrasted, sometimes being successful [37], sometimes

366 unsuccessful [35], or sometimes inducing improvements in some but not all of the parameters

367 [36]. In addition, BBS evaluates balance in isolated balance-related tasks, and TUG combines a

368 limited set of very simple actions (standing-up, walking and sitting-down). Wii Fit-induced

369 improvements were only observed in BBS score and TUG, in a clinical setting, and were not

370 associated with improvements in self-confidence in balance abilities (no changes in $\mathrm{ABC}$ ),

371 therefore it is unclear whether these improvements can be transferred to activities that occur

372 during daily-life and positively impact the quality of life. The general impressions about Wii Fit

373 interventions are, however, currently positive. Our review should therefore encourage further

374 research in order to assist physiotherapists and health professionals in their decision to

375 incorporate the use of Wii Fit into their treatment regimes. Considering that contrasted

376 observations do exist, prescribing Wii Fit should still be considered with caution. 
379 It is unsurprising that Wii Fit has been the object of much attention among physical therapists.

380 The specificities of the Wii Fit games taken together with the technical features of the Wii

381 Balance Board tend to promote medial-lateral and anterior-posterior movements, mimicking

382 exercises that are commonly used in physical rehabilitation programs $[2,47,96]$. The board is

383 composed of multiple pressure sensors able to work together to follow the displacement of the

384 vertical projection of the center of gravity on the floor. Moreover, the device has been validated

385 against the "gold standard" laboratory-grade force platform for assessing standing balance [1]. In

386 addition, high levels of adherence have frequently been reported in the reviewed studies (Table 3

$387 \& 4)$. One may therefore hypothesize that key features of the Wii Fit are the ludic elements that

388 promote adherence in individuals who are not interested in traditional training programs.

389 However, Deutsch et al. emphasizes one limitation of the Wii Fit, which favors the "knowledge

390 of results" rather than the "knowledge of performance" model, i.e. subjects focus on scores

391 rather than on the quality of movements [41]. This is an important finding, since this would limit

392 the relevance of using Wii Fit at home without the supervision of a therapist checking the quality

393 of movement. One difference with the traditional proprioceptive rehabilitation material is that the

394 Wii Balance Board is unable to tilt. Medial-lateral and anterior-posterior displacements are the

395 result of exteroceptive adaptive mechanisms triggered by visual and auditory feedback stimuli

396 that depend on the game scenario.

\section{Limitations}

399 Firstly, the sub-analyses performed in patients included various pathologies. This was

400 highlighted by the high level of heterogeneity between studies in the Wii Fit vs. traditional

401 therapy meta-analyses (see $I^{2}$ in Fig 4B and 5B). While the overall meta-analyses described a 
402 positive effect, the results cannot be predictive of Wii Fit intervention-related changes in one

403 specific population. This emphasizes the requirement for more research in order to determine the 404 optimum usage of Wii Fit for each medical domain. Secondly, the attention given to AVG and 405 other virtual reality devices for the purpose of promoting health has been constantly growing, 406 even after the screening period of this review (July 2009-June 2015). Therefore we encourage 407 readers to also review the new literature on the subject.

Conclusions

410 Originally designed as a ludic health and fitness promotion software, the Wii Fit series grabbed 411 the attention of physical therapists due to the panel of features favoring body movements. Initial 412 promising observations encouraged physicians, from various medical fields, to test the Wii Fit 413 software on numerous populations. The literature still remains contrasted on the preventive 414 effects of Wii Fit on chronic diseases. However, Wii Fit interventions were shown to be effective 415 for the improvement of health status in various types of patients (chronic obstructive pulmonary disease, hemodialysis, renal complications, diabetes, cancer, etc.). Our review identified that the

417 most notable focus of Wii Fit interventions were balance training. The Wii Fit has indeed been 418 successfully used to prevent falls or to induce functional improvements in a wide range of 419 healthy or pathologic populations (e.g. seniors, subjects with neurodegenerative diseases, 420 orthopedic patients, children with developmental delay, multiple sclerosis patients, etc). Our

421 meta-analysis supports the general positive impressions about Wii Fit, suggesting promising 422 applications in a wide range of medical fields. The unexpected entry of a video game into the 423 health device market could create innovative healthcare strategies, however, more research is 424 required to validate these claims. 


\section{References}

426

427

428

429

430

431

432

433

434

435

436

437

438

439

440

441

442

443

444

445

446

447

448

449

450

451

452

453

454

455

456

457

458

459

460

461

462

463

464

465

466

467

468

469

1. Clark RA, Bryant AL, Pua Y, McCrory P, Bennell K, Hunt M. Validity and reliability of the Nintendo Wii Balance Board for assessment of standing balance. Gait Posture. 2010;31(3):307-10. Epub 2009/12/17. doi: 10.1016/j.gaitpost.2009.11.012. PubMed PMID: 20005112.

2. Levac D, Pierrynowski MR, Canestraro M, Gurr L, Leonard L, Neeley C. Exploring children's movement characteristics during virtual reality video game play. Human movement science. 2010;29(6):1023-38.

3. LeBlanc AG, Chaput JP, McFarlane A, Colley RC, Thivel D, Biddle SJ, Maddison R, Leatherdale ST, Tremblay MS. Active video games and health indicators in children and youth: a systematic review. PLoS One. 2013;8(6):e65351. Epub 2013/06/27. doi:

10.1371/journal.pone.0065351. PubMed PMID: 23799008; PubMed Central PMCID: PMCPMC3683002.

4. Peng W, Crouse JC, Lin JH. Using active video games for physical activity promotion: a systematic review of the current state of research. Health Educ Behav. 2013;40(2):171-92. Epub 2012/07/10. doi: 10.1177/1090198112444956. PubMed PMID: 22773597.

5. Morone G, Tramontano M, Iosa M, Shofany J, Iemma A, Musicco M, Paolucci S, Caltagirone $\mathrm{C}$. The efficacy of balance training with video game-based therapy in subacute stroke patients: a randomized controlled trial. BioMed research international. 2014;2014:580861. Epub 2014/05/31. doi: 10.1155/2014/580861. PubMed PMID: 24877116; PubMed Central PMCID: PMC4026958.

6. Wall T, Feinn R, Chui K, Cheng MS. The effects of the Nintendo Wii Fit on gait, balance, and quality of life in individuals with incomplete spinal cord injury. The journal of spinal cord medicine. 2015. Epub 2015/01/24. doi: 10.1179/2045772314y.0000000296. PubMed PMID: 25613853.

7. Bieryla KA, Dold NM. Feasibility of Wii Fit training to improve clinical measures of balance in older adults. Clin Interv Aging. 2013;8:775-81. Epub 2013/07/10. doi: 10.2147/cia.s46164. PubMed PMID: 23836967; PubMed Central PMCID: PMCPMC3699053. 8. Janssen S, Tange H, Arends R. A preliminary study on the effectiveness of exergame Nintendo "Wii Fit Plus" on the balance of nursing home residents. GAMES FOR HEALTH: Research, Development, and Clinical Applications. 2013;2(2):89-95. 9. Rendon AA, Lohman EB, Thorpe D, Johnson EG, Medina E, Bradley B. The effect of virtual reality gaming on dynamic balance in older adults. Age and ageing. 2012;41(4):549-52. 10. Bainbridge E, Bevans S, Keeley B, Oriel K. The effects of the Nintendo Wii Fit on community-dwelling older adults with perceived balance deficits: A pilot study. Physical \& Occupational Therapy in Geriatrics. 2011;29(2):126-35.

11. Fung V, Ho A, Shaffer J, Chung E, Gomez M. Use of Nintendo Wii Fit ${ }^{\mathrm{TM}}$ in the rehabilitation of outpatients following total knee replacement: a preliminary randomised controlled trial. Physiotherapy. 2012;98(3):183-8.

12. Salem Y, Gropack SJ, Coffin D, Godwin EM. Effectiveness of a low-cost virtual reality system for children with developmental delay: a preliminary randomised single-blind controlled trial. Physiotherapy. 2012;98(3):189-95.

13. Liberati A, Altman DG, Tetzlaff J, Mulrow C, Gotzsche PC, Ioannidis JPA, Clarke M, Devereaux PJ, Kleijnen J, Moher D. The PRISMA statement for reporting systematic reviews and meta-analyses of studies that evaluate health care interventions: explanation and elaboration. 
470 PLoS medicine. 2009;6(7):e1000100. Epub 2009/07/22. doi: 10.1371/journal.pmed.1000100.

471 PubMed PMID: 19621070; PubMed Central PMCID: PMC2707010.

472 14. Powell LE, Myers AM. The Activities-specific Balance Confidence (ABC) Scale. J

473 Gerontol A Biol Sci Med Sci. 1995;50A(1):M28-34. Epub 1995/01/01. PubMed PMID: 7814786.

474 15. Berg K. Measuring balance in the elderly: preliminary development of an instrument.

475 Physiotherapy Canada. 1989;41(6):304-11.

476 16. Podsiadlo D, Richardson S. The timed "Up \& Go": a test of basic functional mobility for

477 frail elderly persons. J Am Geriatr Soc. 1991;39(2):142-8. Epub 1991/02/01. PubMed PMID:

4781991946.

479 17. Barcala L, Grecco LAC, Colella F, Lucareli PRG, Salgado ASI, Oliveira CS. Visual 480 biofeedback balance training using wii fit after stroke: a randomized controlled trial. Journal of 481 physical therapy science. 2013;25(8):1027.

482 18. Daniel K. Wii-Hab for Pre-Frail Older Adults. Rehabilitation Nursing. 2012;37(4):195-

483201.

484 19. Yatar GI, Yildirim SA. Wii Fit balance training or progressive balance training in 485 patients with chronic stroke: a randomised controlled trial. J Phys Ther Sci. 2015;27(4):1145-51.

486 Epub 2015/05/23. doi: 10.1589/jpts.27.1145. PubMed PMID: 25995576; PubMed Central

487 PMCID: PMC4433997.

488 20. Follmann D, Elliott P, Suh I, Cutler J. Variance imputation for overviews of clinical trials 489 with continuous response. Journal of clinical epidemiology. 1992;45(7):769-73.

490 21. Meldrum D, Herdman S, Vance R, Murray D, Malone K, Duffy D, Glennon A, McConn-

491 Walsh R. Effectiveness of conventional versus virtual reality-based balance exercises in

492 vestibular rehabilitation for unilateral peripheral vestibular loss: results of a randomized

493 controlled trial. Archives of physical medicine and rehabilitation. 2015;96(7):1319-28 e1. Epub

494 2015/04/07. doi: 10.1016/j.apmr.2015.02.032. PubMed PMID: 25842051.

495 22. Gras LZ, Hummer AD, Hine ER. RELIABILITY AND VALIDITY OF THE

496 NINTENDO WII FIT. Journal of CyberTherapy \& Rehabilitation (JCR). 2009;2(4).

497 23. Graves LE, Ridgers ND, Williams K, Stratton G, Atkinson GT. The physiological cost

498 and enjoyment of Wii Fit in adolescents, young adults, and older adults. Journal of physical

499 activity \& health. 2010;7(3):393-401.

500 24. Owens SG, Garner III JC, Loftin JM, van Blerk N, Ermin K. Changes in physical activity

501 and fitness after 3 months of home Wii Fit ${ }^{\mathrm{TM}}$ use. The Journal of Strength \& Conditioning

502 Research. 2011;25(11):3191-7.

503 25. White K, Schofield G, Kilding AE. Energy expended by boys playing active video games. 504 Journal of science and medicine in sport. 2011;14(2):130-4.

505 26. O'Donovan C, Roche E, Hussey J. The energy cost of playing active video games in 506 children with obesity and children of a healthy weight. Pediatric obesity. 2014;9(4):310-7.

507 27. Ferguson G, Jelsma D, Jelsma J, Smits-Engelsman B. The efficacy of two task-orientated 508 interventions for children with Developmental Coordination Disorder: Neuromotor Task

509 Training and Nintendo Wii Fit training. Research in developmental disabilities.

$510 \quad 2013 ; 34(9): 2449-61$.

511 28. Hammond J, Jones V, Hill EL, Green D, Male I. An investigation of the impact of regular

512 use of the Wii Fit to improve motor and psychosocial outcomes in children with movement

513 difficulties: a pilot study. Child: care, health and development. 2014;40(2):165-75.

514 29. Mombarg R, Jelsma D, Hartman E. Effect of Wii-intervention on balance of children

515 with poor motor performance. Research in developmental disabilities. 2013;34(9):2996-3003. 
516 30. Jelsma D, Geuze RH, Mombarg R, Smits-Engelsman BC. The impact of Wii Fit

517 intervention on dynamic balance control in children with probable Developmental Coordination

518 Disorder and balance problems. Hum Mov Sci. 2014;33:404-18. Epub 2014/01/22. doi:

519 10.1016/j.humov.2013.12.007. PubMed PMID: 24444657.

520 31. Esposito M, Ruberto M, Gimigliano F, Marotta R, Gallai B, Parisi L, Lavano SM,

521 Roccella M, Carotenuto M. Effectiveness and safety of Nintendo Wii Fit Plus ${ }^{\mathrm{TM}}$ training in

522 children with migraine without aura: a preliminary study. Neuropsychiatric disease and treatment.

$5232013 ; 9: 1803$.

524 32. Qualls KK, Arnold SH, McEwen IR, Jeffries LM. Exercise Using the Wii Fit Plus with a

525 Child with Primary Raynaud's Disease and Obesity: A Case Report. Physical \& occupational

526 therapy in pediatrics. 2013;33(3):327-41.

527 33. O'Donovan C, Greally P, Canny G, McNally P, Hussey J. Active video games as an

528 exercise tool for children with cystic fibrosis. Journal of Cystic Fibrosis. 2014;13(3):341-6.

529 34. del Corral T, Percegona J, Seborga M, Rabinovich RA, Vilaro J. Physiological response

530 during activity programs using Wii-based video games in patients with cystic fibrosis (CF).

531 Journal of cystic fibrosis : official journal of the European Cystic Fibrosis Society.

532 2014;13(6):706-11. Epub 2014/06/18. doi: 10.1016/j.jcf.2014.05.004. PubMed PMID: 24935613.

533 35. Ramstrand N, Lygnegård F. Can balance in children with cerebral palsy improve through

534 use of an activity promoting computer game? Technology and Health Care. 2012;20(6):531-40.

535 36. Jelsma J, Pronk M, Ferguson G, Jelsma-Smit D. The effect of the Nintendo Wii Fit on

536 balance control and gross motor function of children with spastic hemiplegic cerebral palsy.

537 Developmental neurorehabilitation. 2013;16(1):27-37.

538 37. Tarakci D, Ozdincler AR, Tarakci E, Tutuncuoglu F, Ozmen M. Wii-based balance

539 therapy to improve balance function of children with cerebral palsy: a pilot study. Journal of

540 physical therapy science. 2013;25(9):1123.

541 38. Ballaz L, Robert M, Parent A, Prince F, Lemay M. Impaired visually guided weight-

542 shifting ability in children with cerebral palsy. Res Dev Disabil. 2014;35(9):1970-7. Epub

543 2014/05/27. doi: 10.1016/j.ridd.2014.04.019. PubMed PMID: 24858794.

544 39. Getchell N, Miccinello D, Blom M, Morris L, Szaroleta M. Comparing Energy

545 Expenditure in Adolescents With and Without Autism While Playing Nintendo ${ }^{\circledR}$ Wii ${ }^{\mathrm{TM}}$ Games.

546 GAMES FOR HEALTH: Research, Development, and Clinical Applications. 2012;1(1):58-61.

547 41. Miyachi M, Yamamoto K, Ohkawara K, Tanaka S. METs in adults while playing active

548 video games: a metabolic chamber study. Med Sci Sports Exerc. 2010;42(6):1149-53.

549 41. Deutsch JE, Brettler A, Smith C, Welsh J, John R, Guarrera-Bowlby P, Kafri M.

550 Nintendo wii sports and wii fit game analysis, validation, and application to stroke rehabilitation.

551 Topics in stroke rehabilitation. 2011;18(6):701-19.

552 42. Gioftsidou A, Vernadakis N, Malliou P, Batzios S, Sofokleous P, Antoniou P, Kouli O,

553 Tsapralis K, Godolias G. Typical balance exercises or exergames for balance improvement?

554 Journal of back and musculoskeletal rehabilitation. 2013;26(3):299-305.

555 43. Melong C, Keats MR. Comparing the effects of a novel and a traditional proprioceptive

556 balance training program on activity adherence and balance control in a healthy university

557 population: a preliminary study. GAMES FOR HEALTH: Research, Development, and Clinical

558 Applications. 2013;2(5):308-12.

559 44. Douris PC, McDonald B, Vespi F, Kelley NC, Herman L. Comparison between Nintendo

560 Wii Fit aerobics and traditional aerobic exercise in sedentary young adults. The Journal of

561 Strength \& Conditioning Research. 2012;26(4):1052-7. 
562 45. Garn AC, Baker BL, Beasley EK, Solmon MA. What are the benefits of a commercial 563 exergaming platform for college students? Examining physical activity, enjoyment, and future 564 intentions. J Phys Act Health. 2012;9(2):311-8.

565 46. Lyons EJ, Tate DF, Ward DS, Wang X. Energy intake and expenditure during sedentary 566 screen time and motion-controlled video gaming. Am J Clin Nutr. 2012;96(2):234-9. Epub 567 2012/07/05. doi: 10.3945/ajcn.111.028423. PubMed PMID: 22760571; PubMed Central 568 PMCID: PMCPMC3396440.

569 47. Michalski A, Glazebrook C, Martin A, Wong W, Kim A, Moody K, Salbach NM, 570 Steinnagel B, Andrysek J, Torres-Moreno R, Zabjek KF. Assessment of the postural control 571 strategies used to play two Wii Fit ${ }^{\mathrm{TM}}$ videogames. Gait \& posture. 2012;36(3):449-53.

572 48. O'Donovan C, Hussey J. Active video games as a form of exercise and the effect of 573 gaming experience: a preliminary study in healthy young adults. Physiotherapy. 2012;98(3):20557410.

575 49. Griffin M, Shawis T, Impson R, Shanks J, Taylor MJ. Comparing the energy expenditure 576 of Wii Fit ${ }^{\mathrm{TM}}$-based therapy versus traditional physiotherapy. GAMES FOR HEALTH: Research, 577 Development, and Clinical Applications. 2013;2(4):229-34.

578 50. Khan O, Parvaiz A, Vassallo D. Acute hernial strangulation following Wii Fit exercises. 579 Acta chirurgica Belgica. 2012;113(1):58-9.

580 51. Tietäväinen A, Gates FK, Meriläinen A, Mandel JE, Hæggström E. Nintendo® Wii Fit 581 based sleepiness tester detects impairment of postural steadiness due to $24 \mathrm{~h}$ of wakefulness.

582 Medical engineering \& physics. 2013;35(12):1850-3.

583 52. Lee D, Lee S, Park J. Effects of indoor horseback riding and virtual reality exercises on 584 the dynamic balance ability of normal healthy adults. J Phys Ther Sci. 2014;26(12):1903-5. Epub 585 2014/12/30. doi: 10.1589/jpts.26.1903. PubMed PMID: 25540494; PubMed Central PMCID: 586 PMC4273054.

587 53. Monteiro-Junior RS, Figueiredo LF, Conceicao I, Carvalho C, Lattari E, Mura G, 588 Machado S, da Silva EB. Hemodynamic responses of unfit healthy women at a training session 589 with nintendo wii: a possible impact on the general well-being. Clinical practice and 590 epidemiology in mental health : CP \& EMH. 2014;10:172-5. Epub 2015/01/24. doi: 591 10.2174/1745017901410010172. PubMed PMID: 25614754; PubMed Central PMCID: 592 PMC4296470.

593 54. Park J, Lee D, Lee S. Effect of virtual reality exercise using the nintendo wii fit on 594 muscle activities of the trunk and lower extremities of normal adults. J Phys Ther Sci. 595 2014;26(2):271-3. Epub 2014/03/22. doi: 10.1589/jpts.26.271. PubMed PMID: 24648647; 596 PubMed Central PMCID: PMC3944304.

597 55. Tripette J, Murakami H, Ando T, Kawakami R, Tanaka N, Tanaka S, Miyachi M. Wii Fit $598 \mathrm{U}$ intensity and enjoyment in adults. BMC research notes. 2014;7(1):567.

599 56. Xian Y, Kakinami L, Peterson ED, Mustian KM, Fernandez ID. Will Nintendo "Wii Fit" 600 Get You Fit? An Evaluation of the Energy Expenditure from Active-Play Videogames. GAMES 601 FOR HEALTH: Research, Development, and Clinical Applications. 2014;3(2):86-91.

602 57. Cone BL, Levy SS, Goble DJ. Wii Fit exer-game training improves sensory weighting 603 and dynamic balance in healthy young adults. Gait Posture. 2015;41(2):711-5. Epub 2015/02/24. 604 doi: 10.1016/j.gaitpost.2015.01.030. PubMed PMID: 25703183.

605 58. Naumann T, Kindermann S, Joch M, Munzert J, Reiser M. No transfer between 606 conditions in balance training regimes relying on tasks with different postural demands: 
607 Specificity effects of two different serious games. Gait Posture. 2015;41(3):774-9. Epub 608 2015/03/21. doi: 10.1016/j.gaitpost.2015.02.003. PubMed PMID: 25791870.

609 59. Cummings J, Duncan E. Changes in affect and future exercise intentions as a result of 610 exposure to a regular exercise programme using the Wii Fit. Sport and Exercise Psychology

611 Review. 2010;6:31-41.

612 60. Nitz J, Kuys S, Isles R, Fu S. Is the Wii Fit ${ }^{\mathrm{TM}}$ a new-generation tool for improving 613 balance, health and well-being? A pilot study. Climacteric. 2010;13(5):487-91.

614 61. Jacobs K, Zhu L, Dawes M, Franco J, Huggins A, Igari C, Ranta B, Umez-Eronini A. Wii 615 health: a preliminary study of the health and wellness benefits of Wii Fit on university students. 616 The British Journal of Occupational Therapy. 2011;74(6):262-8.

617 62. Worley JR, Rogers SN, Kraemer RR. Metabolic responses to Wii Fit ${ }^{\mathrm{TM}}$ video games at 618 different game levels. The Journal of Strength \& Conditioning Research. 2011;25(3):689-93.

619 63. Steenstrup B, Giralte F, Bakker E, Grise P. [Evaluation of the electromyography activity 620 of pelvic floor muscle during postural exercises using the Wii Fit Plus(C). Analysis and perspectives in rehabilitation]. Progres en urologie : journal de l'Association francaise d'urologie et de la Societe francaise d'urologie. 2014;24(17):1099-105. Epub 2014/12/03. doi:

623 10.1016/j.purol.2014.09.046. PubMed PMID: 25450755.

624 64. Tripette J, Murakami H, Gando Y, Kawakami R, Sasaki A, Hanawa S, Hirosako A, 625 Miyachi M. Home-based active video games to promote weight loss during the postpartum 626 period. Med Sci Sports Exerc. 2014;46(3):472-8.

627 65. Guderian B, Borreson L, Sletten L, Cable K, Stecker T, Probst M, Dalleck LC. The cardiovascular and metabolic responses to Wii Fit video game playing in middle-aged and older adults. The Journal of sports medicine and physical fitness. 2010;50(4):436-42. 66. Mullins NM, Tessmer KA, McCarroll ML, Peppel BP. Physiological and perceptual responses to Nintendo ${ }^{\circledR}$ Wii Fit ${ }^{\mathrm{TM}}$ in young and older adults. International Journal of Exercise Science. 2012;5(1):9.

633 67. Reger GM, Holloway KM, Edwards J, Edwards-Stewart A. Importance of Patient Culture and Exergaming Design for Clinical Populations: A Case Series on Exercise Adherence in Soldiers with Depression. GAMES FOR HEALTH: Research, Development, and Clinical 636 Applications. 2012;1(4):312-8.

637 68. Cutter CJ, Schottenfeld RS, Moore BA, Ball SA, Beitel M, Savant JD, StultsKolehmainen MA, Doucette C, Barry DT. A pilot trial of a videogame-based exercise program for methadone maintained patients. Journal of substance abuse treatment. 2014;47(4):299-305. Epub 2014/07/12. doi: 10.1016/j.jsat.2014.05.007. PubMed PMID: 25012555; PubMed Central PMCID: PMC4487635.

642 69. Yuen HK, Holthaus K, Kamen DL, Sword D, Breland HL. Using Wii Fit to reduce 643 fatigue among African American women with systemic lupus erythematosus: A pilot study. 644 Lupus. 2011;20(12):1293-9.

645 70. Yuen HK, Breland HL, Vogtle LK, Holthaus K, Kamen DL, Sword D. The process 646 associated with motivation of a home-based Wii Fit exercise program among sedentary African 647 American women with systemic lupus erythematosus. Disability and health journal. $648 \quad 2013 ; 6(1): 63-8$.

649 71. Meldrum D, Glennon A, Herdman S, Murray D, McConn-Walsh R. Virtual reality 650 rehabilitation of balance: assessment of the usability of the Nintendo Wii ${ }^{\circledR}$ Fit Plus. Disability 651 and rehabilitation: assistive technology. 2012;7(3):205-10. 
652 72. Baltaci G, Harput G, Haksever B, Ulusoy B, Ozer H. Comparison between Nintendo Wii 653 Fit and conventional rehabilitation on functional performance outcomes after hamstring anterior 654 cruciate ligament reconstruction: prospective, randomized, controlled, double-blind clinical trial. 655 Knee Surgery, Sports Traumatology, Arthroscopy. 2013;21(4):880-7.

656 73. Wikstrom EA. Validity and reliability of Nintendo Wii Fit balance scores. Journal of 657 athletic training. 2012;47(3):306.

658 74. Sims J, Cosby N, Saliba EN, Hertel J, Saliba SA. Exergaming and static postural control 659 in individuals with a history of lower limb injury. Journal of athletic training. 2013;48(3):314.

660 75. Punt IM, Ziltener JL, Monnin D, Allet L. Wii Fit exercise therapy for the rehabilitation of 661 ankle sprains: Its effect compared with physical therapy or no functional exercises at all.

662 Scandinavian journal of medicine \& science in sports. 2015. Epub 2015/06/17. doi:

663 10.1111/sms.12509. PubMed PMID: 26076737.

664 76. Miller CA, Hayes DM, Dye K, Johnson C, Meyers J. Using the Nintendo Wii Fit and 665 body weight support to improve aerobic capacity, balance, gait ability, and fear of falling: two 666 case reports. Journal of geriatric physical therapy. 2012;35(2):95-104.

667 77. Albores J, Marolda C, Haggerty M, Gerstenhaber B, ZuWallack R. The use of a home 668 exercise program based on a computer system in patients with chronic obstructive pulmonary 669 disease. Journal of cardiopulmonary rehabilitation and prevention. 2013;33(1):47-52.

670 78. Kempf K, Martin S. Autonomous exercise game use improves metabolic control and 671 quality of life in type 2 diabetes patients-a randomized controlled trial. BMC endocrine disorders. 672 2013;13(1):57.

673 79. Cho H, Sohng KY. The effect of a virtual reality exercise program on physical fitness, 674 body composition, and fatigue in hemodialysis patients. J Phys Ther Sci. 2014;26(10):1661-5. 675 Epub 2014/11/05. doi: 10.1589/jpts.26.1661. PubMed PMID: 25364137; PubMed Central 676 PMCID: PMC4210422.

677 80. Kim SS, Min WK, Kim JH, Lee BH. The Effects of VR-based Wii Fit Yoga on Physical 678 Function in Middle-aged Female LBP Patients. J Phys Ther Sci. 2014;26(4):549-52. Epub 679 2014/04/26. doi: 10.1589/jpts.26.549. PubMed PMID: 24764631; PubMed Central PMCID: 680 PMC3996419.

681 81. Brichetto G, Spallarossa P, de Carvalho MLL, Battaglia MA. The effect of Nintendo® 682 Wii ${ }^{\circledR}$ on balance in people with multiple sclerosis: a pilot randomized control study. Multiple 683 Sclerosis Journal. 2013;19(9):1219-21.

684 82. Nilsagård YE, Forsberg AS, von Koch L. Balance exercise for persons with multiple 685 sclerosis using Wii games: a randomised, controlled multi-centre study. Multiple Sclerosis 686 Journal. 2013;19(2):209-16.

687 83. Plow M, Finlayson M. A qualitative study exploring the usability of Nintendo Wii Fit 688 among persons with multiple sclerosis. Occupational therapy international. 2014;21(1):21-32. 689 84. Prosperini L, Fortuna D, Gianni C, Leonardi L, Marchetti MR, Pozzilli C. Home-based 690 balance training using the Wii balance board: a randomized, crossover pilot study in multiple 691 sclerosis. Neurorehabilitation and neural repair. 2013;27(6):516-25. Epub 2013/03/13. doi: 692 10.1177/1545968313478484. PubMed PMID: 23478168.

693 85. Forsberg A, Nilsagard Y, Bostrom K. Perceptions of using videogames in rehabilitation: 694 a dual perspective of people with multiple sclerosis and physiotherapists. Disability and 695 rehabilitation. 2015;37(4):338-44. Epub 2014/05/17. doi: 10.3109/09638288.2014.918196.

696 PubMed PMID: 24833535; PubMed Central PMCID: PMC4364246. 
697 86. Robinson J, Dixon J, Macsween A, van Schaik P, Martin D. The effects of exergaming 698 on balance, gait, technology acceptance and flow experience in people with multiple sclerosis: a

699

700

701

702

703

704

705

706

707

708

709

710

711

712

713

714

715

716

717

718

719

720

721

722

723

724

725

726

727

728

729

730

731

732

733

734

735

736

737

738

739

740 randomized controlled trial. BMC sports science, medicine and rehabilitation. 2015;7:8. Epub 2015/05/15. doi: 10.1186/s13102-015-0001-1. PubMed PMID: 25969739; PubMed Central PMCID: PMC4427959.

87. Hoffman AJ, Brintnall RA, Brown JK, von Eye A, Jones LW, Alderink G, Ritz-Holland D, Enter M, Patzelt LH, VanOtteren GM. Too sick not to exercise: using a 6-week, home-based exercise intervention for cancer-related fatigue self-management for postsurgical non-small cell lung cancer patients. Cancer nursing. 2013;36(3):175-88.

88. Hoffman AJ, Brintnall RA, Brown JK, von Eye A, Jones LW, Alderink G, Ritz-Holland D, Enter M, Patzelt LH, VanOtteren GM. Virtual reality bringing a new reality to postthoracotomy lung cancer patients via a home-based exercise intervention targeting fatigue while undergoing adjuvant treatment. Cancer nursing. 2014;37(1):23-33.

89. Bower KJ, Clark RA, McGinley JL, Martin CL, Miller KJ. Clinical feasibility of the Nintendo Wii ${ }^{\mathrm{TM}}$ for balance training post-stroke: a phase II randomized controlled trial in an inpatient setting. Clinical rehabilitation. 2014:0269215514527597.

90. Hung JW, Chou CX, Hsieh YW, Wu WC, Yu MY, Chen PC, Chang HF, Ding SE.

Randomized comparison trial of balance training by using exergaming and conventional weightshift therapy in patients with chronic stroke. Archives of physical medicine and rehabilitation. 2014;95(9):1629-37. Epub 2014/05/28. doi: 10.1016/j.apmr.2014.04.029. PubMed PMID: 24862764.

91. Subramaniam S, Wan-Ying Hui-Chan C, Bhatt T. A cognitive-balance control training paradigm using wii fit to reduce fall risk in chronic stroke survivors. Journal of neurologic physical therapy : JNPT. 2014;38(4):216-25. Epub 2014/09/10. doi:

10.1097/npt.0000000000000056. PubMed PMID: 25198867.

92. Omiyale O, Crowell CR, Madhavan S. Effect of Wii-based balance training on corticomotor excitability post stroke. Journal of motor behavior. 2015;47(3):190-200. Epub 2014/11/27. doi: 10.1080/00222895.2014.971699. PubMed PMID: 25425118.

93. Williams B, Doherty NL, Bender A, Mattox H, Tibbs JR. The effect of Nintendo Wii on balance: A pilot study supporting the use of the Wii in occupational therapy for the well elderly. Occupational therapy in health care. 2011;25(2-3):131-9.

94. Yamada M, Aoyama T, Nakamura M, Tanaka B, Nagai K, Tatematsu N, Uemura K, Nakamura T, Tsuboyama T, Ichihashi N. The reliability and preliminary validity of game-based fall risk assessment in community-dwelling older adults. Geriatric Nursing. 2011;32(3):188-94.

95. Bateni H. Changes in balance in older adults based on use of physical therapy vs the Wii Fit gaming system: a preliminary study. Physiotherapy. 2012;98(3):211-6.

96. Duclos C, Miéville C, Gagnon D, Leclerc C. Dynamic stability requirements during gait and standing exergames on the Wii Fit system in the elderly. J Neuroeng Rehabil. 2012;9:28.

97. Franco JR, Jacobs K, Inzerillo C, Kluzik J. The effect of the Nintendo Wii Fit and exercise in improving balance and quality of life in community dwelling elders. Technology and Health Care. 2012;20(2):95-115.

98. Orsega-Smith E, Davis J, Slavish K, Gimbutas L. Wii Fit balance intervention in community-dwelling older adults. GAMES FOR HEALTH: Research, Development, and Clinical Applications. 2012;1(6):431-5. 
741 99. Toulotte C, Toursel C, Olivier N. Wii Fit ${ }^{\circledR}$ training vs. Adapted Physical Activities: 742 which one is the most appropriate to improve the balance of independent senior subjects? A 743 randomized controlled study. Clinical rehabilitation. 2012;26(9):827-35.

744 100. Chao Y-Y, Scherer YK, Wu Y-W, Lucke KT, Montgomery CA. The feasibility of an 745 intervention combining self-efficacy theory and Wii Fit exergames in assisted living residents: A 746 pilot study. Geriatric Nursing. 2013;34(5):377-82.

747 101. Chao YY, Scherer YK, Montgomery CA, Wu YW, Lucke KT. Physical and Psychosocial 748 Effects of Wii Fit Exergames Use in Assisted Living Residents: A Pilot Study. Clinical nursing 749 research. 2014. Epub 2014/12/10. doi: 10.1177/1054773814562880. PubMed PMID: 25488422. 750 102. Cho GH, Hwangbo G, Shin HS. The Effects of Virtual Reality-based Balance Training 751 on Balance of the Elderly. J Phys Ther Sci. 2014;26(4):615-7. Epub 2014/04/26. doi:

752 10.1589/jpts.26.615. PubMed PMID: 24764645; PubMed Central PMCID: PMC3996433.

753 103. Taylor MJ, Lindsverk H, Nygaard M, Hunt J, Shanks J, Griffin M, Shawis T, Impson R.

754 Comparing the energy expenditure of Wii-Fit-based therapy with that of traditional

755 physiotherapy in an older adult population. J Am Geriatr Soc. 2014;62(1):203-5. Epub

756 2014/09/03. PubMed PMID: 25180391.

757 104. Chao YY, Lucke KT, Scherer YK, Montgomery CA. Understanding the Wii Exergames

758 Use: Voices from Assisted Living Residents. Rehabil Nurs. 2015;14(10):216.

759 105. Nicholson VP, McKean M, Lowe J, Fawcett C, Burkett B. Six weeks of unsupervised 760 Nintendo Wii Fit gaming is effective at improving balance in independent older adults. Journal 761 of aging and physical activity. 2015;23(1):153-8. Epub 2014/03/05. doi: 10.1123/japa.2013-0148. 762 PubMed PMID: 24589631.

763 106. Roopchand-Martin S, McLean R, Gordon C, Nelson G. Balance Training with Wii Fit 764 Plus for Community-Dwelling Persons 60 Years and Older. Games Health J. 2015;4(3):247-52. 765 Epub 2015/07/17. doi: 10.1089/g4h.2014.0070. PubMed PMID: 26182070.

766 107. Pigford T, Andrews AW. Feasibility and benefit of using the Nintendo Wii Fit for 767 balance rehabilitation in an elderly patient experiencing recurrent falls. Journal of student 768 physical therapy research. 2010;2(1):12-20.

769 108. Williams MA, Soiza RL, Jenkinson AM, Stewart A. EXercising with Computers in Later 770 Life (EXCELL)-pilot and feasibility study of the acceptability of the Nintendo ${ }^{\circledR}$ WiiFit in 771 community-dwelling fallers. BMC research notes. 2010;3(1):238.

772 109. Agmon M, Perry CK, Phelan E, Demiris G, Nguyen HQ. A pilot study of Wii Fit

773 exergames to improve balance in older adults. Journal of Geriatric Physical Therapy.

774 2011;34(4):161-7.

775 110. Chan TC, Chan F, Shea YF, Lin OY, Luk JKH, Chan FHW. Interactive virtual reality

776 Wii in geriatric day hospital: A study to assess its feasibility, acceptability and efficacy.

777 Geriatrics \& gerontology international. 2012;12(4):714-21.

778 111. Jorgensen MG, Laessoe U, Hendriksen C, Nielsen OBF, Aagaard P. Efficacy of Nintendo 779 Wii training on mechanical leg muscle function and postural balance in community-dwelling 780 older adults: a randomized controlled trial. The Journals of Gerontology Series A: Biological 781 Sciences and Medical Sciences. 2013;68(7):845-52.

782 112. Padala KP, Padala PR, Burke WJ. WII-FIT AS AN ADJUNCT FOR MILD 783 COGNITIVE IMPAIRMENT: CLINICAL PERSPECTIVES. Journal of the American Geriatrics 784 Society. 2011;59(5):932-3. 
785

786

787

788

789

790

791

792

793

794

795

796

797

798

799

800

801

802

803

804

805

806

807

808

809

810

811

812

813

814

815

816

817

818

819

820

821
113. Esculier J-F, Vaudrin J, Beriault P, Gagnon K, Tremblay LE. Home-based balance training programme using Wii Fit with balance board for Parkinson's disease: a pilot study. Journal of rehabilitation medicine. 2012;44(2):144-50.

114. dos Santos Mendes FA, Pompeu JE, Lobo AM, da Silva KG, de Paula Oliveira T, Peterson Zomignani A, Pimentel Piemonte ME. Motor learning, retention and transfer after virtual-reality-based training in Parkinson's disease-effect of motor and cognitive demands of games: a longitudinal, controlled clinical study. Physiotherapy. 2012;98(3):217-23.

115. Padala KP, Padala PR, Malloy TR, Geske JA, Dubbert PM, Dennis RA, Garner KK, Bopp MM, Burke WJ, Sullivan DH. Wii-Fit for improving gait and balance in an assisted living facility: a pilot study. Journal of aging research. 2012;2012.

116. Pompeu JE, dos Santos Mendes FA, da Silva KG, Lobo AM, de Paula Oliveira T, Peterson Zomignani A, Pimentel Piemonte Maria Elisa. Effect of Nintendo Wii ${ }^{\text {TM}}$-based motor and cognitive training on activities of daily living in patients with Parkinson's disease: A randomised clinical trial. Physiotherapy. 2012;98(3):196-204.

117. Mhatre PV, Vilares I, Stibb SM, Albert MV, Pickering L, Marciniak CM, Kording K, Toledo S. Wii Fit balance board playing improves balance and gait in Parkinson disease. Pm\&r. 2013;5(9):769-77.

118. Esculier J-F, Vaudrin J, Tremblay LE. Corticomotor Excitability in Parkinson's Disease During Observation, Imagery and Imitation of Action: Effects of Rehabilitation Using Wii Fit and Comparison to Healthy Controls. Journal of Parkinson's disease. 2014;4(1):67-75.

119. Goncalves GB, Leite MA, Orsini M, Pereira JS. Effects of using the nintendo wii fit plus platform in the sensorimotor training of gait disorders in Parkinson's disease. Neurology international. 2014;6(1):5048. Epub 2014/04/20. doi: 10.4081/ni.2014.5048. PubMed PMID: 24744845; PubMed Central PMCID: PMC3980145.

120. Liao YY, Yang YR, Cheng SJ, Wu YR, Fuh JL, Wang RY. Virtual Reality-Based

Training to Improve Obstacle-Crossing Performance and Dynamic Balance in Patients With Parkinson's Disease. Neurorehabilitation and neural repair. 2015;29(7):658-67. Epub 2014/12/30. doi: 10.1177/1545968314562111. PubMed PMID: 25539782.

120. Laver K, Ratcliffe J, George S, Burgess L, Crotty M. Is the Nintendo Wii Fit really acceptable to older people?: a discrete choice experiment. BMC geriatrics. 2011;11(1):64.

121. Hakim RM, Salvo CJ, Balent A, Keyasko M, McGlynn D. Case report: a balance training program using the Nintendo Wii Fit to reduce fall risk in an older adult with bilateral peripheral neuropathy. Physiother Theory Pract. 2015;31(2):130-9. Epub 2014/12/18. doi: 10.3109/09593985.2014.971923. PubMed PMID: 25515202.

123. Peng W, Lin JH, Crouse J. Is playing exergames really exercising? A meta-analysis of energy expenditure in active video games. Cyberpsychol Behav Soc Netw. 2011;14(11):681-8. Epub 2011/06/15. doi: 10.1089/cyber.2010.0578. PubMed PMID: 21668370. 